\title{
Evolutionary Engineering of Complex Functional Networks
}

\author{
vorgelegt von \\ Diplom-Physiker \\ Pablo F. Kaluza \\ Von der Fakultät II - Mathematik- und Naturwissenschaften \\ der Technischen Universität Berlin \\ zur Erlangung des akademischen Grades \\ Doktor der Naturwissenchaft \\ - Dr. rer. nat. - \\ genehmigte Dissertation
}

Promotionsausschuss:

Vorsitzender: Prof. Dr. E. Sedlmayr

Gutachter: Prof. Dr. H. Engel

Gutachter: Prof. Dr. A. S. Mikhailov

Gutachter: Prof. Dr. M. Vingron

Tag der wissenschaftlichen Aussprache: 20.11.2007

Berlin 2007

D 83 


\section{Abstract}

Complex biological networks are responsible for many fundamental processes in living organisms, including signal transduction and genetic expression in biological cells and signal processing in neural networks. These functional networks, a product of the evolution, are characterized by their robustness against damages, mutations and noise. Functionality and robustness are reflected in their architectures which exhibit structures different from random networks and lattices.

To design functional networks, robust against local damages and noise, and to study, how the requirements of functionality and robustness are reflected in their architecture, are the objectives of this work. Our studies are performed using a model of flow distribution networks representing a simplification of biological signal transduction systems. In the model, networks process signals (fluxes) passing from input to output nodes, and generate output patterns which define the function of a network.

The design of networks with prescribed functions (output patterns) is performed by using an evolutionary process of mutations and selections. Furthermore, not only functionality, but also the requirements of different kinds of robustness are imposed in the network design. Three criteria of robustness are considered: robustness against the deletion of randomly chosen nodes or links and robustness against distributed noise.

Remarkable differences between architectures of the designed networks, depending on the criteria of robustness imposed during their construction, are observed. Particularly, motif distributions of these networks are different. Comparing them with real biological networks, we have found that the networks robust against deletion of links show motif distributions which are very similar to those of some neural systems and to the development transcription and signal transduction networks of biological macroorganisms.

An evolutionary process, similar to that used to construct robust networks, has been employed in a reverse engineering problem. Using it, we have constructed networks with given Laplacian spectra. We have demostrated that construction of networks with a prescribed Laplacian spectrum is possible. Statistical structural properties of families of cospectral graphs have been analyzed. 


\section{Zusammenfassung}

Viele fundamentale Prozesse in lebenden Organismen, darunter Signaltransduktion und Genexpression in biologischen Zellen und Signalverarbeitung in neuronalen Netzen, laufen in komplexen biologischen Netzwerken ab. Charakteristisch für diese funktionalen Netzwerke, die im Laufe der Evolution entstanden sind, ist ihre Robustheit gegen Beschädigungen, Mutationen und Rauschen. Funktionalität und Robustheit spiegeln sich wider in ihren Architekturen, die Strukturen zeigen, welche sich sowohl von zufälligen Netzwerken als auch von (regelmässigen) Gittern unterscheiden.

Funktionale Netzwerke zu entwerfen, die robust sind gegen lokale Beschädigungen und Rauschen sowie zu untersuchen, wie die Anforderungen von Robustheit und Funktionalität in ihrer Architektur reflektiert wird, ist das Ziel dieser Arbeit. Dazu wird ein Flussverteilungsnetzwerkmodell betrachtet, das eine Vereinfachung biologischer Signaltransduktionssysteme darstellt. In diesem Modell verarbeiten die Netzwerke von den Eingangs- zu den Ausgangsknoten laufende Signale (Flüsse) und erzeugen Ausgabemuster, die die Funktion eines Netzwerks definieren.

Netzwerke mit vorgebenen Funktionen werden durch einen evolutionären Prozess aus Mutationen und Selektionen erzeugt. Bei diesem Prozess wird nicht nur die Funktionalität des Netzwerks vorgegeben, sondern es werden auch die Anforderungen an die verschiedenen Arten von Robustheit definiert. Drei Kriterien für Robustheit werden betrachtet: Robustheit gegen die zufällige Auslassung von Knoten oder Verbindungen und die Robustheit gegenüber verteiltem Rauschen.

Abhängig von den Vorgaben für Robustheit während des Erzeugungsprozesses, lassen sich bemerkenswerte Unterschiede in der Architektur der Netzwerke beobachten. Besonders treten diese Unterschiede bei den Motivverteilungen der erzeugten Netzwerke hervor. Im Vergleich mit realen biologischen Netzwerken stellte sich eine starke Ähnlichkeit der Motivverteilungen derjenigen Netzwerke, die robust gegen die Auslassung von Verbindungen sind, zu denen einiger neuronaler Systeme sowie zu Signaltransduktionsnetzwerken und development transcription in biologischen Makroorganismen heraus.

Ein evolutionärer Prozess, ähnlich dem bei der Konstruktion robuster Netzwerke verwendeten, wurde zur Lösung eines Reverse-Engineering-Problems benutzt. Mit seiner Hilfe wurden Netzwerke mit gegebenen Laplace-Spektren erzeugt. Es wurde gezeigt, dass es möglich ist, Netzwerke mit einem vorgegebenen Laplace-Spektrum zu konstruieren. Statistische Struktureigenschaften von Familien kospektraler Graphen wurden analysiert. 


\section{Contents}

Contents $\quad$ v

$\begin{array}{ll}\text { Introduction } & 1\end{array}$

1 Background 5

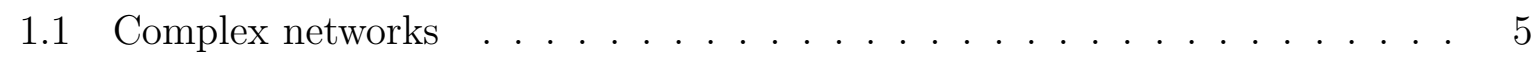

1.1.1 Network organization . . . . . . . . . . . . . 6

1.1.2 Dynamics, evolution and robustness . . . . . . . . . . . . 7

1.2 Biological networks . . . . . . . . . . . . . . . . . . . . . . . 9 9

1.2.1 The neural network of Caenorhabditis elegans . . . . . . . . . 9

1.2.2 Signal transduction networks . . . . . . . . . . . . . . . . . . 10

1.2.3 Genetic expression networks . . . . . . . . . . . . . . . 14

1.2.4 Robustness of biological systems . . . . . . . . . . . . . . 18

1.3 Network properties . . . . . . . . . . . . . . . . 20

1.3.1 Structural properties . . . . . . . . . . . . . 20

1.3.2 Motif distributions . . . . . . . . . . . . . . . 23

1.4 Network superfamilies . . . . . . . . . . . . . . . . . . 24

2 Flow distribution networks $\quad 27$

2.1 The pipeline network model . . . . . . . . . . . . . . 27

2.1.1 Network topology . . . . . . . . . . . . . . . . . . . . . . . . 28

2.1.2 Network operation . . . . . . . . . . . . . . . . . . . . . . . . 29

2.1.3 Algebraic aspects ........................ 31

2.2 Input-output relationships . . . . . . . . . . . . . . . . 32

3 Evolutionary design of flow distribution networks with prescribed out- 
put patterns $\quad 35$

3.1 Evolutionary design method . . . . . . . . . . . . . 35

3.1 .1 Optimization algorithm . . . . . . . . . . . 36

3.1 .2 Evolutionary mutations . . . . . . . . . . . 36

3.1.3 Example of network construction . . . . . . . . . . . . 38

3.1.4 Comparison of two mutation schemes . . . . . . . . . . . . . . 39

3.1.5 The temperature parameter . . . . . . . . . . . . . . 40

3.2 Optimization statistics . . . . . . . . . . . . . . . . . 40

3.2 .1 Flow error . . . . . . . . . . . . . . . . . . 42

3.2.2 Structural statistical properties of designed networks . . . . . . . 43

3.2.3 Motif distributions . . . . . . . . . . . . . . . . . . . 47

4 Functional networks robust against local damage $\quad 51$

4.1 Definition of robustness . . . . . . . . . . . . . . . . . . . . . 51

4.2 Evolutionary optimization . . . . . . . . . . . . . . 52

4.2.1 Optimization algorithm . . . . . . . . . . . . . 52

4.2.2 Ensembles of networks . . . . . . . . . . . . . . . . 53

4.2.3 Temperature parameters $\sigma_{E}$ and $\sigma_{R} \ldots \ldots \ldots \ldots 5$

4.3 Statistical properties of designed robust networks . . . . . . . . . . . 55

4.3 .1 Distribution of flow errors . . . . . . . . . . . . . . 56

4.3 .2 Mean robustness . . . . . . . . . . . . . . . . 58

4.3.3 Structural statistical properties of robust networks . . . . . . . . . 59

4.3.4 Motif distributions of robust networks . . . . . . . . . . . 63

5 Functional networks robust against noise $\quad 67$

5.1 Noise in flow distribution networks . . . . . . . . . . . . 67

5.1.1 Definition of topological noise . . . . . . . . . . . . 67

5.1 .2 Definition of noise robustness . . . . . . . . . . . . . . 68

5.2 Evolutionary design of networks robust against noise . . . . . . . . . 71

5.2 .1 Optimization algorithm . . . . . . . . . . . . . . 71

5.2 .2 Example of construction of a robust network . . . . . . . . . . . 72

5.2 .3 Temperature parameter . . . . . . . . . . . . . 72

5.3 Statistical properties of designed networks . . . . . . . . . . . . 73 
5.3.1 Flow error of designed networks . . . . . . . . . . . . . 74

5.3.2 Robustness of designed networks . . . . . . . . . . . . . 75

5.3.3 Structural statistical properties of designed networks . . . . . . . 77

5.3 .4 Motif distributions . . . . . . . . . . . . . . 80

5.4 Comparison of cross-robustness of various designed networks . . . . . . . . 82

6 Evolutionary construction of networks with required Laplacian spectra 85

6.1 Laplacian spectra and relaxation phenomena in chemical networks . . . . . 85

6.2 Evolutionary construction method . . . . . . . . . . . . 86

6.2.1 Spectral density and spectral distance . . . . . . . . . . . 87

6.2.2 Evolutionary optimization algorithm . . . . . . . . . . 88

6.2.3 Evolutionary mutations . . . . . . . . . . . . . . 88

6.2.4 Example of spectral reconstruction . . . . . . . . . . . . . 90

6.3 Construction of families of cospectral graphs . . . . . . . . . . . . . . . 92

6.3.1 Structural statistical properties of cospectral graphs . . . . . . . . . 92

6.3.2 Comparison with non-directed graphs . . . . . . . . . . . . . 94

6.4 Origin of cospectral directed graphs . . . . . . . . . . . . . . 95

$\begin{array}{lr}\text { Conclusions } & 97\end{array}$

$\begin{array}{ll}\text { Bibliography } & 103\end{array}$ 


\section{Introduction}

Complex network organization is found in many biological and artificial systems. The main characteristic of such systems is the presence of selective interactions between their elements which form a complicated architecture. The biological examples are cross-coupled reaction systems in a cell, such as signal transduction and gene expression networks, and the neural networks. Among artificial systems, we find the Internet, WWW, logistic distribution networks and networks of interacting machines in industrial production systems. It is known that biological networks show remarkable properties of robustness with respect to local damages, mutations and noise. They can perform well their functions despite a relatively high degree of damages inside some tolerance windows. It is very desirable to reproduce such properties in the artificial systems.

How biological systems are designed by the evolution to perform their functions? How their functions are reflected in their architecture? How does the special structure of such networks make them robust against mutations and noise? These are the principal questions that must be addressed. These general questions can be studied from different points of view. For particular biological systems, a large amount of work has been done to model in detail their structure, elements and interactions. Detailed models of interactions between proteins in signal transductions networks are available and network structures by which they interact are known. Realistic models of neurons are also available. With these models, one can try to find the unknown parameters and produce a dynamics which matches with the behaiviour of a real system.

There is, however, evidence that many properties of network systems are not strongly dependent on the operation details of individual elememts, but are mainly determined by the architecture of a network. Network architectures are studied in terms of the characteristics of graphs constructed by taking as nodes and connections the elements and the interactions of real systems. Such statistical properties of networks as the distribution of the number of nodes with a particular number of connections (degree distribution), the density of interconnections between the neighbors (clustering coefficient) and average distances between nodes can be considered. These properties of real networks have been found to show important differences with respect to those of random networks or regular lattices. Real networks often have degree distributions with long tails, in contrast to random graphs with the Poisson distributions. The small-world property, i. e. the possibility to connect any two nodes of a network through only a few steps, is characteristic 
for many real systems. The differences are seen not only in these classical structural properties. Frequencies of the appearance of small subgraphs (such as different motifs with three nodes) may deviate remarkably in real networks from their randomized versions.

In the above approach, network systems are investigated without considering their functions. Then, robustness of such systems is analyzed in terms of purely structural criteria. For example, damage in the network connections can lead to its disintegration into small disconnected subnetworks. Although this approach may be useful, it does not allow to study how damages and noise affect particular performances of the systems. The requirements of robustness in networks cannot be fully understood without considering their functions.

We want to address these questions from another point of view. Using a simple model, we will construct many networks with prescribed functions, which are robust against different kinds of damages or against noise. Because all such networks have the same function, effects of different kinds of robustness on the network architecture can be statistically investigated. Through a convergent evolution, different systems may find similar solutions to face the same problem. Artificially constructed networks, which have functions similar to those of the real systems, can also display similar solutions. Note that this approach is different from reverse engineering where one tries to reconstruct an existing system from its set of data. It can be seen as a complement of the detailed study and of the modeling of particular real networks.

The simple model, used in our investigations, is an abstraction of signal transduction networks of a biological cell. In this model, propagating signals are represented by fluxes. These fluxes are redistributed by the middle nodes. When a flux is applied to an input node, it reaches in varying fractions different output nodes. The set of responses to the activation of different input nodes defines the function of such a network. The network operation, which specifies processing of input signals, is formulated in terms of distributions of fluxes and it depends only on the network architecture.

Working with such flow distribution networks, we demonstrate that it is possible to construct functional networks with prescribed input-output relationships by using an evolutionary process of optimization. With this optimization process, networks can be designed not only to have a prescribed function, but also to be robust against local damages, such as removal of a node or a link, and against structural noise. The designed networks display remarkable structural differences depending on the criteria of optimization considered in their evolutions, even when they have been made to evolve using the same algorithm and the same scheme of evolutionary mutations. This indicates that the profound differences in the network architectures are mainly related to the criteria of robustness imposed. The comparision with real systems, in terms of their motif distributions, reveals that our networks, optimized to be robust against the removal of a link, have a structure similar to signal transduction networks of a cell and to the neural network of the $C$. elegans. On the other hand, networks optimized against the removal of a node and against noise possess motif distributions which do not agree with any known biological 
system.

The thesis is organized in six chapters. In the first chapter, principal characteristics of complex networks are introduced. We consider three examples of biological networks: the neural networks of $C$. elegans, the signal transduction system of a cell and gene expresion networks. Different models used in their studies are considered and several examples of robustness found in such systems are discussed. Finally, we present the tools which we will further use in the analysis of the network architecture of our designed networks. Three structural properties are introduced: the degree distribution, the clustering coefficient, and the measure of path lengths in networks. Another structural property, the motif distribution, is discussed separately, because it plays a particular role in our analysis.

Chapters 2, 3, 4, and 5 are dedicated to the construction of networks with prescribed functions, which are further optimized against different kinds of damages and noise. The model of flow distribution network is presented in Chapter 2. There, the main characteristics of the model are shown and its architecture, operation and input-output relationship are defined. The prescribed input-output relationship (i. e. its target input-output pattern) is discussed in relation to the real systems previously shown and its random structure is presented. The flow error of a network, the difference between its actual input-output pattern and the target input-output pattern, is defined.

In Chapter 3, networks are designed to perform a prescribed input-output relationship. The network design is performed by using an evolutionary optimization process of mutations and selections. This process is based on a variant of the Metropolis algorithm known in statistical physics. Two schemes of evolutionary mutations are employed. One is based on changing single links in each mutation and the other is based on addition (or subtraction) of a randomly chosen subnetwork to the network which is evolving. Different aspects of the evolutionary algorithm are considered. Structural properties of the designed networks and their motif distributions are studied as function of the number of middle nodes, for different schemes of evolutionary mutations used.

The concept of robustness in the model of flow distribution networks is introduced in Chapter 4. Two possible kinds of damages which may affect the operation of the networks are considered: deletion of a randomly chosen node and deletion of a randomly chosen connection. Now, the aims of the optimization are not only the reduction of the flow error, but also an increase in the robustness of networks. The evolutionary algorithm is modified to combine these two criteria, switching between them. If the flow error of the network is larger than the threshold, the network is not functional and its flow error must be decreased. On the other hand, if the flow error is smaller than the threshold, the network is functional and its robustness should be increased. Structural properties of designed robust networks are statistically studied as functions of the threshold and of the kind of damage considered during the evolutionary process.

Effects of topological noise are investigated in Chapter 5. The influence of such structural static noise on flow distribution networks, optimized only by flow error, is studied depending on the network size. Then, an evolutionary algorithm, similar to the one used 
in the Chapter 4, is applied to design functional networks robust against noise. Structural properties of designed networks and their dependence on the threshold and noise intensity are considered. Finally, we analyze the cross-robustness, i. e. the degree of noise robustness of the networks designed to be robust against local damages (link and node removals) and vice versa. We show that, generally, networks robust against node removal and noise are not robust against link deletions.

In Chapter 6, a similar evolutionary algorithm is applied to reconstruct the architecture of networks of first-order chemical reactions from the Laplacian spectra, determining concentration dynamics of the involved chemical species. This reverse engineering is performed using an optimization method which minimizes the spectral distance between a target set of Laplacian eigenvalues and that of an evolving network. We show that the construction of a network with a prescribed Laplacian spectrum is possible. However, Laplacian spectra of directed graphs do not uniquely determine the graph structure. Therefore, different cospectral graphs are constructed, by running the evolution with the same Laplacian spectrum. Although the constructed graphs have the same Laplacian spectra, their structural properties are different. We discuss the origin of a large amount of cospectral graphs and its relation to particular graph structures. 


\section{Chapter 1}

\section{Background}

\subsection{Complex networks}

Many biological, social, chemical and technological systems are constituted by similar elements which interact and generate complex global behaviors impossible to be produced by one of these elements separately. Generally, such systems represent networks. Among the biological processes with network organization, signal transductions, gene expressions, chemotaxis, metabolism and the cell cycle can be mentioned. These networks involve cross-coupled chemical reactions with different kinds of molecules. At a larger scale, neural systems represent a classic example of network organization, where neurons are interconnected, processing information and executing complex tasks. Moreover, we can mention ecological networks, with interactions among various species, including predator-prey relationships. Social networks are characterized by different kinds of interactions between individuals, such as, e. g., collaborations among actors or scientists, or human sexual contacts. Human languages generate complex networks, connecting words according to their meanings. As technological and information systems, we can refer to the examples of the Internet, WWW, networks of electrical power supply and transportation in cities, and the networks of interacting machines in the factories. Thus, the elements which form the networks can very much vary.

The way, in which the elements of a network are connected, determines its topology. In the mentioned systems, the topologies are far from being simple. Additionally, interactions between the elements may have their own complex properties which affect the network operation. For example, in a network of chemical reactions, nodes can represent chemical species and a connection can be a particular reaction between two of them. However, each interaction is further characterized by a rate constant which specifies the speed of the reaction. In a neural network, connections are synapses and they can be excitatory or inhibitory. Additionally, each synaptic connection has its own strength, which is specific for any pair of neurons. In the Internet networks, information flows between the computers. Thus, fluxes are established through connections with certain capacities, 
which determine the maximum achievable fluxes of information. Finally, in transportation systems, not only a maximum transport capacity of a link, but also the distance between the nodes (the distribution centers) is important.

\subsubsection{Network organization}

Given the complexity that these systems present, there are several different approaches for their study. One approach is to study only topologies of networks $[1,2,3]$, ignoring operations of their elements and details of their interactions. Then, a network is represented by a graph where a connection between two nodes indicates only the existence of the respective interaction. For example, in a graph of a production network of a factory [14], each machine would be a node and the presence of a production flow between two machines would be a link. In the case of the WWW, each node is a website and a hyperlink is a connection. In a neural network, nodes are neurons and a link between two nodes is present if a synaptic connection between them exists. If networks are fully connected, but interconnections are characterized by certain weights, we can choose some threshold and put a link in its graph if the strength of the respective interaction is larger than the threshold.

In this approach, many details of the networks are neglected and attention is focused on the patterns of interactions between the elements. To characterize network topologies, such structural properties as the degree distribution, the clustering coefficient and the path lengths are used. Moreover, the presence of particular subgraphs or motifs, such as feedback or feedforward loops, can be analyzed.

Structural properties are traditionally used in the studies of the topology of network systems. The degree distribution tells how frequently the nodes with a given number of connections are found in a graph. The mean degree is the average number of connections per node in the graph. The clustering coefficient of a node shows how many potential links among the neighbors of a node are actually present in the graph. The clustering coefficient of the whole network is the average of those of its nodes. In social networks, this structural property indicates how often our friends would be also friends between them [4]. The path between two nodes is the sum of the links which connect them. The length of the shortest possible path defines the distance between two nodes. The mean path length of a network is the average of the distances between all its pairs of nodes.

In real networks, the structural properties may differ remarkably from those of random graphs or lattices. There are many ways to construct random graphs, but the most simple construction was proposed by Erdös-Rényi [5]. To obtain a random Erdös-Rényi graph, each pair of nodes is connected with the same probability $p$. The probability that a node has exactly $k$ connections is given by a binomial distribution, and the degree distribution is $N P_{N-1, p}(k)$. Its mean value, the mean degree $D$ of the network, is $p(N-1)$. In many investigations of random graphs, the limit when $N \rightarrow \infty$ is considered. In this case, one takes $p \rightarrow 0$ while keeping the mean degree $p(N-1)$ constant. This leads to a 
Poissonian degree distribution. For this reason, such graphs are also known as Poisson random graphs. In a lattice, all nodes have the same degree. Moreover, nodes of a lattice are ordered periodically in space and they are connected only with their nearest neighbors.

Most of the real large networks show degree distributions significantly deviating from the Poisson distribution of random graphs. Often, such distributions have long tails, following power laws $P(k) \sim k^{\gamma}$ for high degrees $k$. This indicates the existence of hubs. The networks with power-law degree distributions are known as scale-free networks. A theory, proposed by Barabási and Albert [6], explains this property of real networks taking into account that nodes shows preferential attachment to the nodes with a high degree. For example, in the WWW, new web pages are usually connected with already existing important pages that have many connections, because they are easy to find. The clustering coefficients of real networks are also often different from those of random networks. Real networks show considerably larger values of clustering coefficients with respect to the random networks with the same size and the same number of connections.

Another important characteristic of large real networks is their small-world property. Path lengths between two nodes in such a network are often short. In social networks, this is reflected in the six-degrees-of-separation-concept. Assuming that two persons are connected if they know each other personally, it has been shown that it is possible to connect any pair of persons in the US through only six persons on the average. This is really remarkable if we consider the size of the respective network, with the population of about 350 millons people. The small-world property, which is present also in some random graphs, is not found in lattices. In a lattice, the average distance between the nodes increases proportionally to the lattice size. Watts and Strogatz [4] have proposed a model of random small-world networks which interpolate between an ordered finitedimentional lattice and a random graph.

\subsubsection{Dynamics, evolution and robustness}

The second important approach to study network systems is based on their dynamics. Generation of global signals, synchronization or desynchronization of the whole networks or parts of them (clusters) and other kinds of dynamical behaviour are then considered. To study such properties, one needs to model in detail the behaviors of the nodes and the connections among them. For example, in a production chain, machines can be modeled as dynamical units and connections between them would include buffers and would be characterized by their distances, speeds of product transportations, maximum capacities, etc. In a signal transduction network of a cell, we must consider the dynamics of chemical reactions involved, their variation with temperature, concentrations, etc. Many systems, where elements show periodic dynamics, have been modeled using the concept of phase oscillators with various kinds of interactions [7]. Using phase oscillator models, it is possible to reproduce many behaviours characteristic for real systems and to understand the conditions needed to produce the synchronization in such networks [8]. 
Today, one of the most important scientific goals is to understand the relationship between the topological organization and the dynamical properties of various real networks. However, in a realistic detailed model it may be difficult to distinguish the effects of topology and to separate them from the effects related to the dynamics of individual elements. To avoid this, one can generate a graph with the topology of a real network and use some simple artificial dynamics over it, based on the fluxes or logic operations (as in the Kauffman networks).

Unlike the artificial systems, biological systems are a result of evolution. Such systems evolve, through natural mutations and selections, in order to optimize their architecture for the performance of particular tasks. The architecture optimization should not only yield good solutions in terms of the required functions. It must generate architectures robust with respect to changes in the environment, to attacks of other organisms, and to the intrinsic noise. Resources of the organisms, such as energy, size, number of nodes and connections, are limited and simple solutions based on trivial redundances and parallelization, can be difficult or impossible to implement. In general, requirements of the task and robustness optimizations of a network compete for the resources and the solution is a compromise between these two kinds of optimization. To discriminate which properties of the network are responsible for its performance (task) and which ones are responsible for its robustness improvement is a delicate problem.

Understanding the relationship between topologies and dynamical properties of natural systems can be important in order to design artificial systems. The natural biological systems show exceptional properties, which are highly desirable to reproduce, including robustness against different kinds of damages, noise and variations in the environment. Generally, robustness implies the persistence of the characteristic system behavior under perturbations or uncertainty [9]. The classical example is provided by neural networks of the brain where large portions can be damaged (destroying neurons and synapses), but the system would still maintain its operations with an acceptable performance. A similar situation is revealed in gene knockout experiments [10]. In these experiments, functions of genes are studied by making some of them inoperative and showing the effects of their absence for the organism. For the yeast Saccharomyces cerevisiae, it has been found by making inoperative one gene per experiment (single knockout analysis), that only $13 \%$ of the genes are essential, so that their absence is lethal for the organism. All other genes are dispensable, demonstrating high genetic robustness.

In such artificial systems as the Internet and WWW, robustness is related to the persistence of connectivity between different parts of a network when a fraction of nodes is deleted [11]. For these systems, it has been shown that random failures of the nodes do not affect seriously the communication in a network, whereas directed attacks to highly connected nodes (hubs) can produce desintegration of the network, when a critical fraction of such nodes is deleted. Usually, there are only a few highly connected nodes which are important for the communication between the rest of the nodes. The existence of a critical fraction of nodes, whose absence leads to the disintegration of a network, has been understood using the percolation theory of random graphs [12]. Problems of optimization 
of such networks against waves of directed and random attacks have been investigated [13].

\subsection{Biological networks}

In this section we briefly consider three kinds of biological systems: the neural network of the nematode $C$. elegans, genetic regulatory networks and signal transduction networks of a cell. These systems show network organization with functional substructures having relatively small sizes, on the order of hundreds of elements. They are much smaller than such networks as the Intenet, WWW or the brain, where millions or billions of elements are present. Their general function is to process certain input information, giving as result the generation of specific signals or the production of some chemical substances.

If we understand the relationship between the structure, the functionality and the robustness of such networks, this knowledge can be applied to important artificial systems which possess similar general functions, such as industrial and logistic transportation systems [14].

\subsubsection{The neural network of Caenorhabditis elegans}

Neural systems are generally characterized by complex architecture and dynamics, as well as by a large number of elements and a high connectivity. This is true for the brain and the nervous system in big animals. However, small biological organisms possess nervous systems with only few neurons and are still capable of performing simple information processing tasks.

The nematode Caenorhabditis elegans is an example of a simple organism with a small neural network. This animal has been intensively studied and the number of cells of its body and their functions are perfectly known. The hermaphrodite $C$. elegans consists of 959 cells, 302 of them being neurons. The complete structure and synaptic connectivity of its nervous system have been determined [15]. Additionally, functional classification of its nervous cells into sensory neurons, inter-neurons, and motor neurons, is available.

This simple neural network is responsible, among other functions, for the chemotaxis, a process by which the nematode finds its food. The network which controls chemotaxis in this animal is known, because the neurons of the sensory organ, the motor cells and the map of connections, have all been determined. However, it is not yet possible to determine what synapses are excitatory or inhibitory. Mathematical models of this neural network have been proposed [16]. The global structure of the network shows three well-defined regions. The network includes input neurons in the sensory organ, which receive external stimuli. The information is further processed in the inter-region. Finally, the results of the information processing are expressed in the output neurons which control muscle cells of the animal. 


\subsubsection{Signal transduction networks}

Signal transductions are processes by which a cell converts one kind of signals or stimuli into another. The processes, referred to as signal transduction, typically involve a sequence of biochemical reactions inside the cell, which are carried out by proteins. The kind of stimuli, which the cell can receive, depends on its environment. For example, molecules may attach to receptor proteins on its surface, its body may be heated or cooled, the cell may be illuminated by light of various wavelengths, mechanically stretched or electrically polarized. The responses to these stimuli also vary. They can represent, for example, activation of genes, production of metabolic energy, cell locomotion, etc.

Because of the importance of signal transduction networks for the cell, their study is a broad field of biology. Many reviews on mathematical models and different aspects of these systems have appeared, including those by Heinrich [17], Vayttaden [18] and Sauro [19]. Below, some typical mathematical models of signal transduction are outlined.

\section{Linear signaling cascade}

The linear signaling cascade is the simplest example of a transduction system. Here, signal processing begins with chemical stimulation of receptors on the surface of a cell or inside of it. This activates production of receptor proteins, followed by a variety

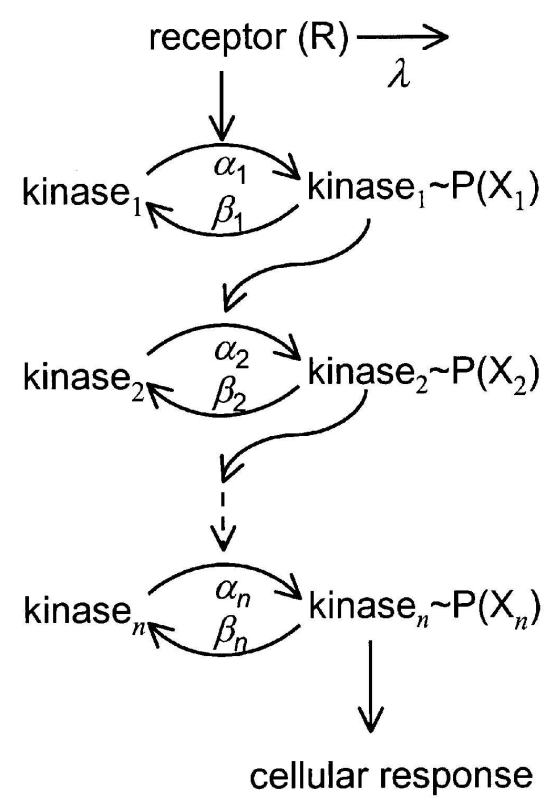

Figure 1.1: Signal transduction cascade. In this scheme, $R$ and $X_{i}$ indicate the activated receptor and the kinases, respectively. $\lambda$ is the rate constant for the inactivation of the receptor, and $\alpha_{1}$ and $\beta_{1}$ are the rates constants for the kinase and phosphatase reactions (R. Heinrich et al. [17]). 
of intracellular signaling proteins that distribute the signal to appropriate parts of the cell. The proteins which are activated depend on the kind of the signal. The cascade of induced reactions is called the pathway of a stimulus. Among intracellular signaling proteins, there are kinases, phosphatases, GTP-binding proteins, and other proteins with which they interact. At the end of each pathway are target proteins, which are altered when the pathway is active and thus change the behaviour of the cell.

In these processes, kinase proteins play a fundamental role, acting as machines used for the catalysis of other kinases. The kinase molecule can have two states: the active state, when it is phosphorylated, and the inactive state, when it is dephosphorylated. When a kinase is activated, it can act as a catalyst and produce phosphorylation of other kinds of kinases. The repetition of such processes generates the pathway. The phosphorylation of a kinase consists of the addition of a phosphate group to its structure and it is catalyzed by a protein kinase. The inverse processe of inactivation of a kinase, the dephosphorylation, is performed by the enzymes called phosphatases, which remove the phosphate group from the kinase.

The scheme of the protein kinase linear signal cascade [17] is shown in Fig. 1.1. The chain of subsequent activations starts with the phosphorylation of the first kinase $X_{1}$ by the stimulated receptor $R$. The output signal of this pathway is the activation of the last kinase $X_{n}$ which produces a specific response. The signals (active kinases) are deactivated by phosphotases, which dephophorylate the kinases, and by the inactivation of the receptor.

The concentration of the active form of kinase $X_{i}$ increases, because it is produced from its inactive form $\widetilde{X}_{i}$ through a phophorylation reaction catalized by the active form of the kinase $X_{i-1}$. If this reaction has the rate constant $\widetilde{\alpha}_{i}$, the increase is given by $\widetilde{\alpha}_{i} \widetilde{X}_{i} X_{i-1}$. On the other hand, the active form of the kinase is deactivated by phosphatase. Assuming that phosphatases are present at a constant concentration, the desphopharylation reaction is a first-order reaction and the concentrations decrease proportional to $\beta_{i} X_{i}$. Thus, the concentration of the active kinase $i$ is governed by the equation,

$$
\frac{d X_{i}}{d t}=\widetilde{\alpha}_{i} \widetilde{X}_{i} X_{i-1}-\beta_{i} X_{i}
$$

Introducing $C_{i}=\widetilde{X}_{i}+X_{i}$ as the total concentration of the kinase $i$ and $\alpha_{i}=\widetilde{\alpha}_{i} C_{i}$ as a pseudo-first order rate constant, equation (1.1) is written in the form

$$
\frac{d X_{i}}{d t}=\alpha_{i} X_{i-1}\left(1-\frac{X_{i}}{C_{i}}\right)-\beta_{i} X_{i}
$$

The first kinase in the cascade is activated by the stimulated receptor. For this kinase, equation (1.2) must be modified as

$$
\frac{d X_{1}}{d t}=\alpha_{1} R(t)\left(1-\frac{X_{1}}{C_{1}}\right)-\beta_{1} X_{1}
$$




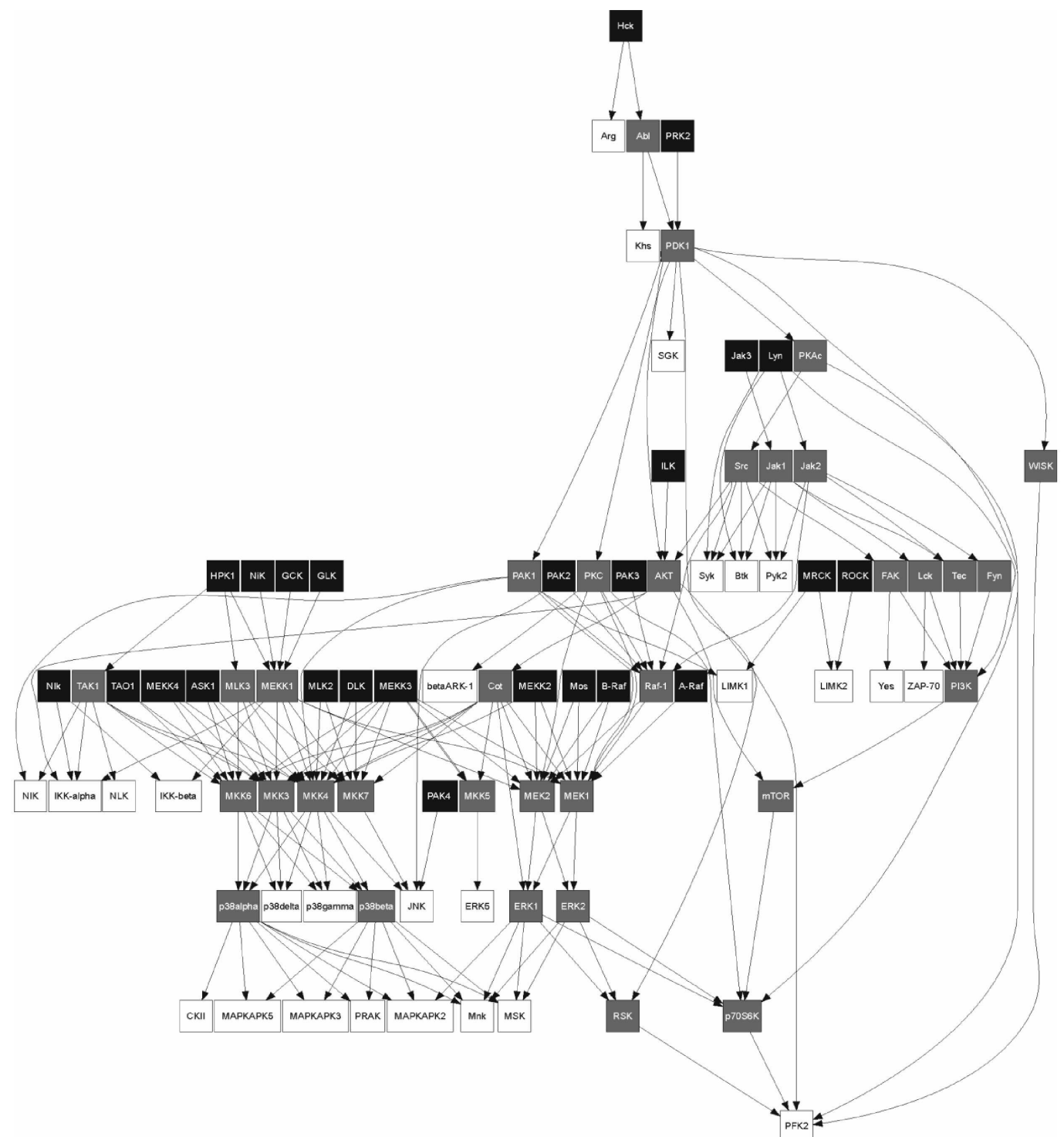

Figure 1.2: Transpath network. Black and white boxes indicate input and output kinases, respectively. Other kinases (gray boxes) receive and transmit signals (from $[20])$.

where $R(t)$ is the intensity of the stimulus. After its stimulation, the activity of the receptor decreases as $R(t)=\exp (-\lambda t)$, where $\lambda$ is the characteristic activation time. Equations (1.2) and (1.3) determine the dynamics of the cascade.

Using this model, several questions can be discussed [17]: How fast does the signal arrive at its destination? How long does the signal last? How strong is the signal?

The model is further simplified for a weakly activated pathway, where the involved kinases are phosphorylated only to a low degree $\left(X_{i} \ll C_{i}\right)$. This situation can occur when the concentration of the activated receptor is low, when the receptor is rapidly inactivated 
and/or when the kinases are present at high concentration. In this case, equations (1.2) become linear

$$
\frac{d X_{i}}{d t}=\alpha_{i} X_{i-1}-\beta_{i} X_{i}
$$

\section{Signaling network}

The signaling process typically involves cross-interaction of many kinases, forming networks where different pathways can be connected by common kinases, or which have the same target proteins. Figure 1.2 shows an example of a signaling network from the Transpath database [20]. Here, documented interactions between proteins (they do not correspond to an in vivo organism) are shown. In the graph, each node corresponds to a kinase and each connection to a reaction of phosphorylation. Many topological properties can be studied for such networks, including the length and the number of cycles, the length of the paths between input and output kinases (which are the numbers of reactions needed to generate a response), and the degree distributions.

The dynamical model for the linear cascade can be modified for the case of a network [21] [22],

$$
\frac{d X_{i}}{d t}=\left(\sum_{j=1}^{n} \alpha_{i j} X_{j}\right)\left(1-\frac{X_{i}}{C_{i}}\right)-\beta_{i} X_{i} .
$$

Here, $\alpha_{i j}$ are the second-order rate constants to the enzymatic reactions of phosphorylation of the kinase $i$ which is catalyzed by the kinaze $j$. By using such models, dynamics of
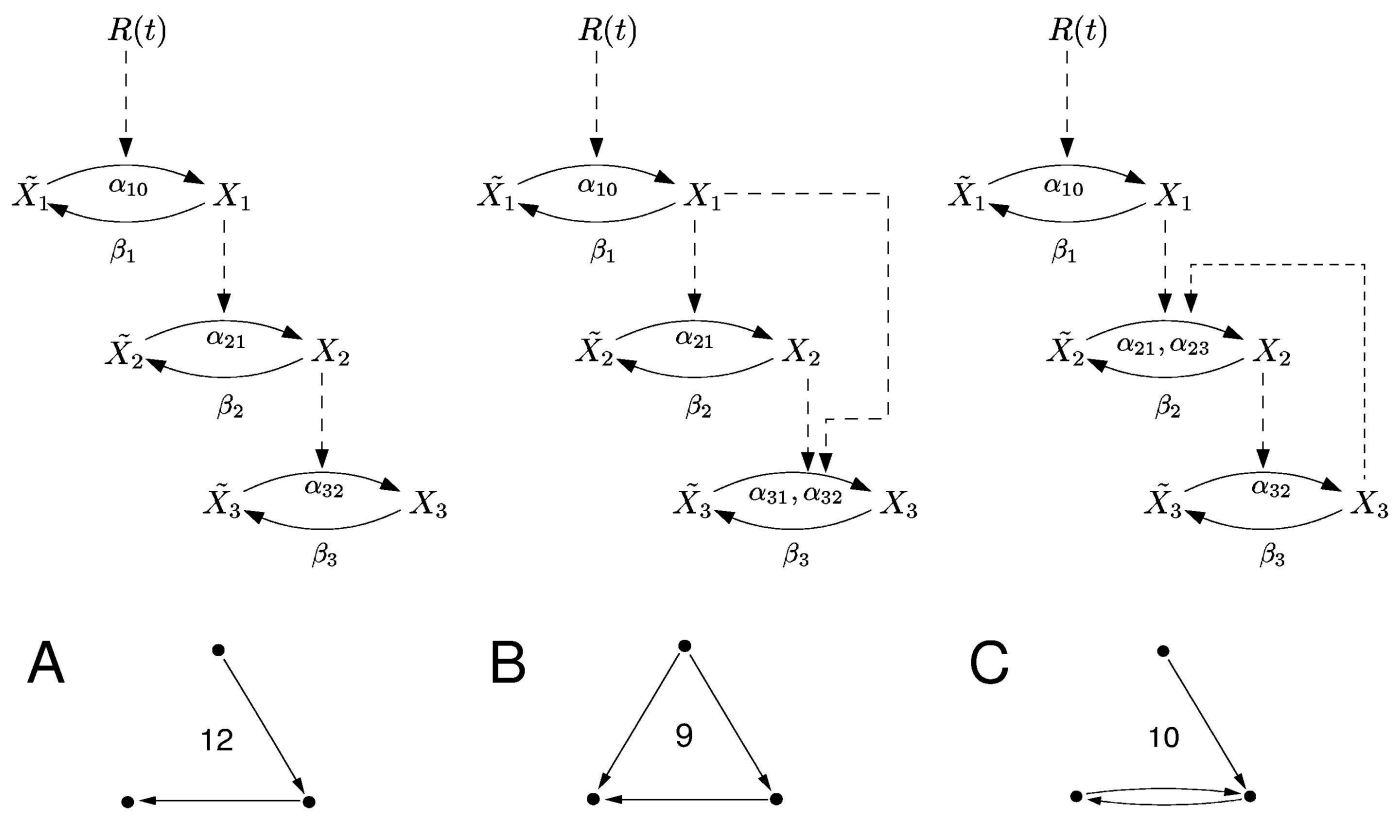

Figure 1.3: Different network structures with three kinases and three phosphotases (from $[21])$. 
actual signal transduction networks can be investigated [21]. Particularly, one can study the effects of various topological structures and feedback loops on the network operation. Several examples of structural network elements are shown in Fig. 1.3.

\subsubsection{Genetic expression networks}

Gene expression is a process by which a cell produces proteins from the instructions encoded into its DNA. This process has three steps (Fig. 1.4). The first step consists in the transcription of a region of the DNA (a gene) into an RNA molecule (also called a transcript). Second, different proteins process the RNA transcript and translate it into a polypeptide chain. Third, additional proteins help to fold and modify this polypeptide chain into a biochemically active protein.

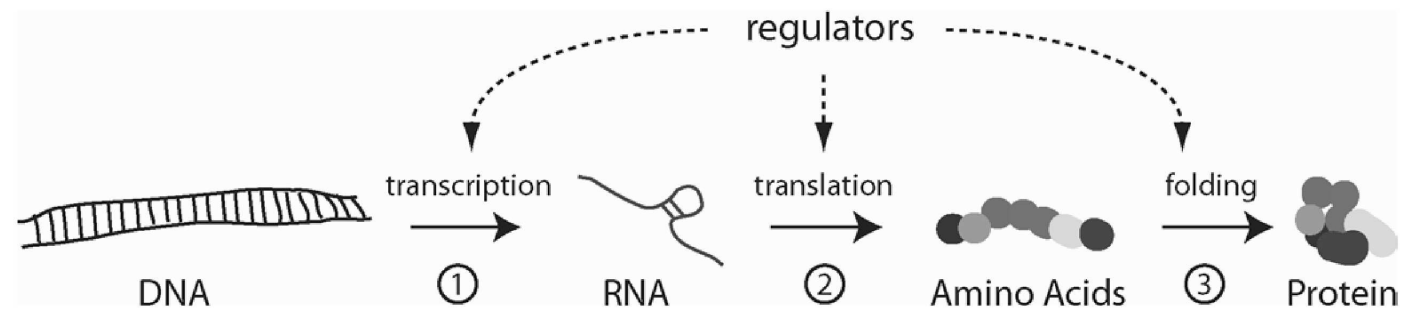

Figure 1.4: Gene expression process. (1) A region of DNA (a gene) is transcribed into RNA. (2) RNA is translated into a chain of amino acids. (3) The amino acid chain is folded into its functional 3-dimensional protein structure. Regulation of cell dynamics can occur at any of these steps. From Gardner and Faith [23].

Regulatory molecules can control the concentration and the form of the product of each step. These regulators are usually fully formed proteins, but any of the intermediate products (RNA, polypeptides, or proteins) also may act as regulators of gene expression. Thus, the production of a specific protein is controlled via the rate of synthesis or the rate of elimination by degradation or modification to another form. Usually, however, degradation of RNA is nonspecific and, therefore, it is not involved in the control.

The synthesis of RNA is performed by RNA polymerase (RNAP), a protein complex that reads the DNA and copies it into RNA. The transcription of the DNA begins when RNAP recognizes a promoter, which is a control sequence of DNA upstream of the transcribed region, and binds to it (Fig. 1.5a). After binding, RNAP opens the DNA double helix and slides along the DNA sequence, elongating the RNA message by adding ribonucleotides that match the DNA sequence. Transcription proceeds until the RNAP encounters a stop sequence (terminator) in the DNA.

The transcription process can be controlled by proteins called transcription factors (TF). A TF binds to a short, unique DNA sequence, called a motif, in or near the promoter. This protein can be an activator or a repressor. An activator increases the 


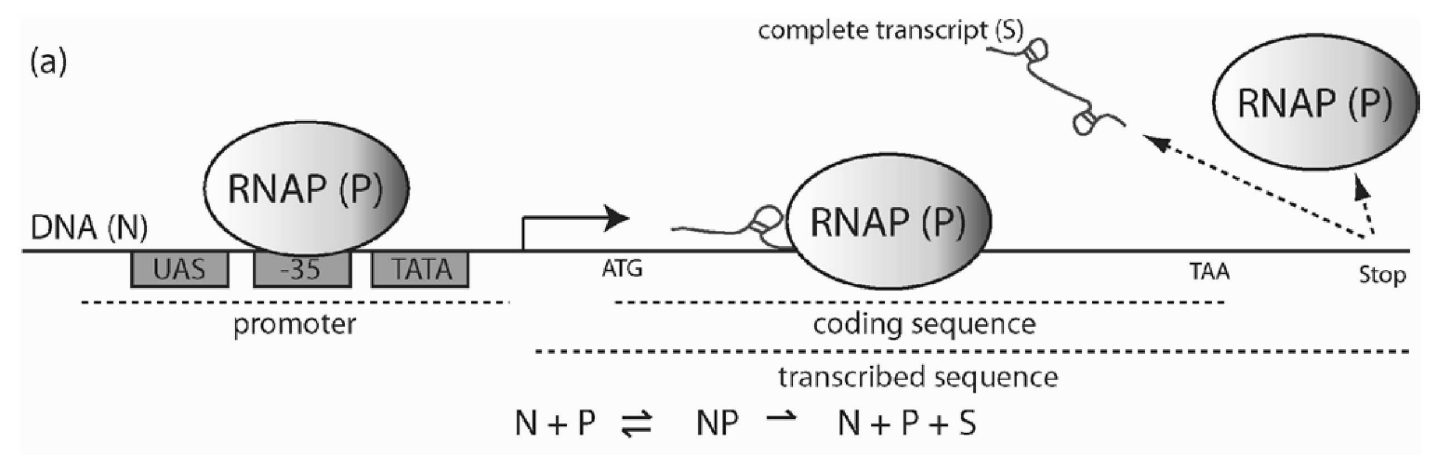

(b)

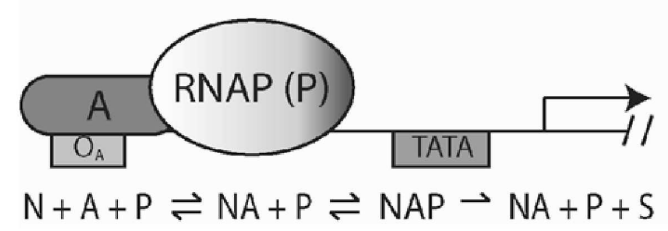

(c)

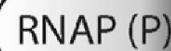

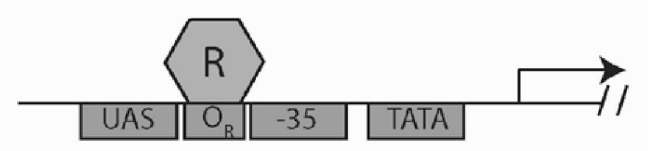

$\mathrm{NR}+\mathrm{P} \rightleftharpoons \mathrm{N}+\mathrm{R}+\mathrm{P} \rightleftharpoons \mathrm{NP}+\mathrm{R} \rightarrow \mathrm{N}+\mathrm{P}+\mathrm{R}+\mathrm{S}$

Figure 1.5: (a) RNA transcription begins when an RNA polymerase (RNAP) binds to upstream recognition sequence on the DNA molecule (promoter). The two strands of DNA are separated and RNAP moves along the DNA, transcribing an RNA copy of it until a stop region on DNA knocks the polymerase off. (b) Some genes have an activator protein that binds to a motif $\left(O_{A}\right)$ near the promoter, increasing the affinity of RNAP for the promoter. (c) Other genes have repressor proteins that bind to motif $\left(O_{R}\right)$ in the promoter, blocking access of RNAP to important regions of the promoter. From Gardner and Faith [23].

affinity of the RNAP (Fig. 1.5b) for the promoter, whereas a repressor protein can act prohibiting binding of the RNAP protein to the DNA (Fig. 1.5c). The transcription factors belong to a larger class of proteins called DNA-binding proteins, whose main characteristic is the possibility to bind to the DNA molecule. The molecules of such kind can be at the end of a pathway of a signal transduction network [24]. Thus, the activation or repression of a gen can be controlled by an external signal.

Mathematical models of gene expresion networks often focus on the transcription process. They aim to model temporal evolution of the concentration of the RNA transcripts. It is possible to obtain a simple model by considering the RNAP $(P)$, the DNA promoter $(N)$, and the RNA transcript $(S)$ as chemical species. Applying the principles of rate-law kinetic modeling, one obtains:

$$
P+N \stackrel{k_{1} k_{2}}{\rightleftarrows} P N \stackrel{k_{3}}{\longrightarrow} P+N+S
$$

where $k_{1}$ and $k_{2}$ describe the on and off rates of RNAP bilding, and $k_{3}$ is the transcript elongation rate. If $k_{3}$ is small or under the assumption that the concentration of the promoter is much smaller than the concentration of RNAP, the differential equation for 
transcription can be written as [23]:

$$
\frac{d s}{d t}=\frac{V_{m} p}{p+K_{m}}-\beta s
$$

where the lowercase letters are concentrations of the respective species, $V_{m}=k_{3} n$ is the maximum rate of synthesis, $K_{m}=\left(k_{2}+k_{3}\right) / k_{1}$ is the Michaelis constant, and $\beta$ is the rate constant of RNA transcript degradation. $K_{m}$ describes the activation threshold for $p$. For concentrations of $p$ above $K_{m}$, the rate of synthesis begins to saturate at its maximum value $V_{m}$. More realistic models have been proposed where the effects of the activators and repressors in the transcription process are considered.

Genes interact (see Fig. 1.6), because the product of a gene can activate or repress other genes. This leads to a network of interactions between genes. There exist mainly

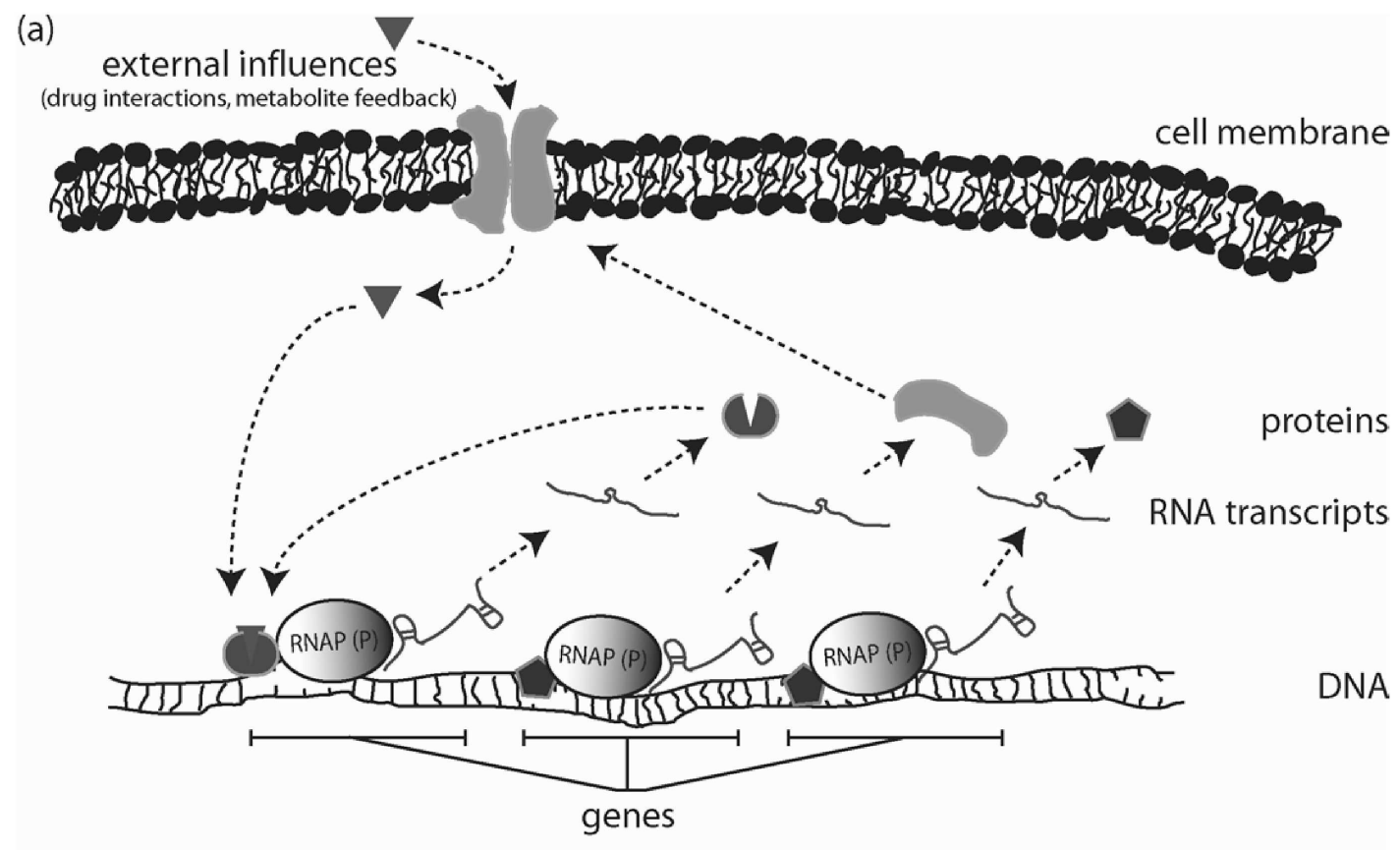

(b)

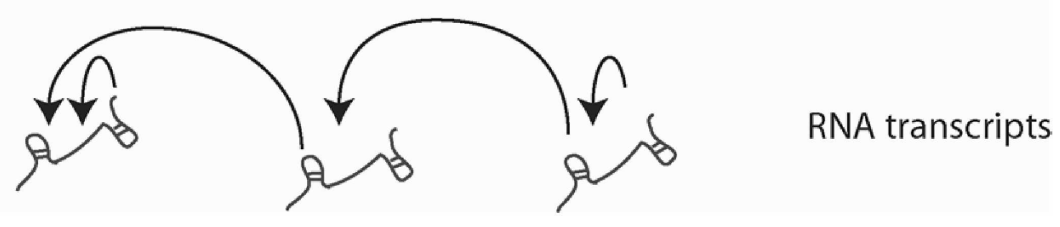

Figure 1.6: Biological networks are regulated at many levels. (a) Example of network where protein transcription factors (proteins in figure) influence the expression of different RNA transcripts. An external metabolite (triangle) is imported and it binds to one of the transcription factors, enabling it to bind DNA and initiate transcription. Part (b) shows the gene network model of the network shown in (a). From Gardner and Faith [23].

two strategies to model such interactions. A "physical" approach [25] seeks to identify 
the protein factors, that regulate transcription, and the DNA motifs, to which the factors bind. In other words, it looks for true physical interactions between regulatory proteins and their promoters.

A second strategy, the "influence" approach, seeks to identify regulatory influences between RNA transcripts. It looks for transcripts that act as inputs, whose concentration changes can explain the changes in output transcripts. An advantage of the influence strategy is that the model can implicitly capture regulatory mechanisms at the protein level that are not physically known. This type of models is sometimes called a gene regulatory network or a gene network. A gene network model does not usually describe physical relationships between regulators and transcripts. Nevertheless, gene network models provide a global view of gene regulation that is not restricted to $\mathrm{TF} /$ promoter interactions.

In general, a gene network model can be represented as a directed graph and, depending on the degree of simplification of cell regulation considered, this graph can be described mathematically as a system of differential equations, as a Boolean network, or as a Bayesian network.

Differential equation models [26] describe a gene networks as a system of differential equations. The rate of change in concentration of a particular transcipt, $x_{i}$, is given by a nonlinear influence function, $f_{i}$, of the concentrations of other RNA species.

$$
\frac{d x_{i}}{d t}=f_{i}\left(x_{1}, \ldots, x_{N}\right)
$$

where $N$ is the number of transcripts in the network. In general, only a small number of RNA species regulate a particular transcript, so that the networks are sparse.

Boolean network models [27] [28] describe transcript levels as binary variables. Here, "1" means that a transcript is expressed, or that it is changed relative to a reference state. "0" means that a transcript is not expressed or unchanged. The next state of a transcript is determined by a Boolean function of the state of the input transcripts,

$$
x_{i}(t+1)=f^{B}\left(x_{1}(t), \ldots, x_{N}(t)\right),
$$

where $f_{i}^{B}$ is a Boolean function for the transcript $i$.

Finally, we mention Bayesian networks models [29], which allow to take into account noise and data limitations inherent in expression studies and also to retain the combinatorial logic of transcription regulation. In the Bayesian networks, the state of a transcript is represented by a random variable, $X_{i}$. This random variable is specified by a probability distribution function, $f_{i}$, which depends on (i. e. conditioned by) a set of regulator transcripts, $X_{j}$. We can write this as:

$$
P\left(X_{i}=x_{i} \mid X_{j}=x_{j}\right)=f_{i}\left(x_{i} \mid x_{j}\right),
$$

where $j=1, \ldots, N$, and $j \neq i$, and a lowercase $x$ denotes a particular value of $X$. The use of the conditional distribution $P\left(X_{i} \mid X_{j}\right)$ implies a further restriction: a transcript may 
be a regulator of another transcript only provided that the network contains no cycles (i. e., no feedback loops). This restriction is the principal limitation of the Bayesian network models.

\subsubsection{Robustness of biological systems}

Biological systems show exceptional properties which are highly desirable to reproduce in artificial systems and, particularly, robustness against different kinds of damages, noise and mutations. In general, robustness means the persistence of the system characteristic behavior under perturbations or conditions of uncertainty [9]. We present below a brief discussion of the origins of noise and mutations in these systems. Then, we show examples of robust biological systems and different approaches to the investigations of biological robustness.

\section{Noise and random variation in biological networks}

Biochemical reaction systems are affected by internal and external noises. Internal noise comes from random fluctuations of stochastic chemical reaction events in finitesize biochemical systems. The intensity of the internal noise is inversely proportional to the square root of the number of particles in the system. Because of the relatively small sizes of cellular or subcellular reaction systems (where some chemical species have populations of only about a hundred molecules), internal noise is important. Such internal fluctuations can be introduced into a model by describing the chemical reaction system, in a microscopic way, as a stochastic process governed by a master equation. A related approach is to use a mesoscopic description, intermediate between the microscopic (master equations) and the continuous (kinetic equations) descriptions and formulated in terms of continuous fluctuating concentrations of reactants. Then, fluctuations caused by the atomistic nature of reactions are modeled by introducing some internal noise into the kinetic equations [30].

External noise originates from random variation of one or more of the externally set control parameters, such as the rate constants associated with a given system of reactions. These variations have an external origin, since we must consider the dynamical system of biochemical reactions as part of some larger system (i. e. of the "environment") with intrinsically chaotic behaviour. Because a small subsystem cannot exhibit any significant backward influence, the action of the fluctuating environment is described as some noise with the given, externally defined, statistical properties. Such noise induces fluctuations in the system parameters. A typical example of the external noise is the variation of the temperature which can modify the rate constants of the involved reactions.

Not only internal and external noises affect these systems. Specific damages can also occur through mutations. For example, a mutation can modify the structure of a protein and reduce the number of its possible interactions with other proteins. In a network of 
interacting proteins, this corresponds to the deletion of a connection between two of them. Mutations may strongly affect a system. An entire gene can be inactivated or a neuron can die in a nervous system.

In genetic systems, noise and mutations in the process of gene expression contribute to the phenotypic variability [31]. Studies of the phenotypic variation in clonal populations have been performed [32], showing how a stochastic process, inherent to biochemical reactions, influences gene regulation.

\section{Robustness in biological systems}

Depiste the presence of strong internal noise, mutations and environmental fluctuations, biological systems can function in a stable and precise way up to some tolerance threshold $[9,33]$.

The complexity of biochemical networks raises the question of the stability of their functioning. One possibility is that to achieve an appropriate function, reaction rate constants and enzymatic concentrations of a network need to be chosen in a very precise manner. But then any deviation from the 'fine-tuned' values would ruin the network performance. Another possibility is that the key properties of biochemical networks are robust; that is, they are relatively insensitive to the precise values of biochemical parameters. This second possibility has been demostrated in the experiments using chemotaxis of Escherichia coli [34], one of the best-characterized sensory systems. It has been proposed [35] that robust adaptation of signal transduction networks is a direct consequence of their architecture and is related to the presence of dynamical feedback loops.

Genetic robustness, i. e. preservation of an optimal phenotype despite genetic mutations, is a fundamental property of living systems incorporated at various levels of biological complexity [36]. Protein tolerance to amino acid substitutions [37], gene dispensability in yeast [38], and error tolerance of complex biological networks [11], are some important examples. The amino acid substitution produces a mutation, which changes the message encoded in the amino acid sequence and, therefore, affects the shape and the function of proteins. When a mutation leads to the inactivation of a gene, because it cannot be transcribed into RNA or translated into a functional protein product, such a mutation is called the null mutation. The role played by the duplicated genes in the robustness against null mutations [39] has been discussed. "Gene dispensability" refers to the fact that the deletion or inactivation of a single gene may often produce only a little effect in the process of gene expression. Robustness against inactivation of genes has been studied through the knockout analysis, where one or several genes are inactivated, and their functions are then inferred by considering changes in the phenotype [10].

The cell-cycle process, by which one cell grows and divides into two daughter cells, has been shown to possess a robust design [40]. In this case, using a simple dynamical model of the network structure of the budding yeast, it was found that one of the attractors of the dynamical system corresponds to the stationary state of the real biological system. The 
robustness in this system is related to the fact that a large number of initial conditions lead to this attractor. Because most of the initial conditions evolve to the attractor, the system is viewed as robust. The large number of initial conditions leading this attractor is still maintained under mutations, such as deletion or addition of connections, or when changes in the functions of the interactions are introduced (from positive regulation to repression and vise versa).

Currently, robustness is seen as an evolutionary principle which directs the evolution of biological systems. Using genetic models of networks with Boolean dynamics [41], a possibility to obtain robust networks through an evolutionary process has been shown. Here, the robustness was the criterion of optimization and was defined as the conservation of the dynamics during the evolution. An obtained network was robust, if its mutated versions had high probability to have the same dynamics. However, the function which the network must perform, i. e. its dynamical attractor, was arbitrary in these studies, so that only robust networks with a non-prescribed function were thus constructed.

Other studies of construction of robust networks through evolution have been performed by Handorf and Heinrich [42]. In these studies, networks evolved from an initial set of reactions by growing through the addition of new compatible reactions which find their substrates in the previous sets of reactions. By analysing the effect of deletions of one or more reactions, the authors draw conclusions on the robustness of the obtained networks. As in the previous example of logical networks, the function of the networks was not retained during the evolution in these investigations.

\subsection{Network properties}

In this section we present formal definitions of several local properties which will be used later. First, classical statistical properties of networks, such as the mean degree, the clustering coefficient, the average path length and the diameter, are introduced. All of these definitions are made considering directed networks, and we indicate when there exists a significant difference with respect to the undirected networks. After that, we introduce a method for the analysis of small local structures in the networks. Such small subnetworks or motifs give us the description of local organization of the networks and they allow us to characterize and compare networks with different origins and sizes.

\subsubsection{Structural properties}

Statistical structural properties, considered below, are traditionally used to describe topologies of the graphs corresponding to real networks and a large amount of literature on these properties is available. Reviews of Albert and Barabasi [1], Newman [2] and Dorogovtsev and Mendes [3] present these properties and give their interpretations depending on the real systems studied. A completely mathematical treatment of such topics 
can be found, for example, in the monograph by Distel [43]. The definitions, which are used by us to characterize the networks that we study, are based on these references.

\section{Degree distribution}

The degree $d$ of a node in a network is the number of connections which touch it. If the connections of a network are directed, it is possible to define separately the indegree $d_{\text {in }}$ and the outdegree $d_{\text {out }}$ of each node, as the number of input and output connections that come to or go from the node, respectively. Obviously, $d=d_{\text {in }}+d_{\text {out }}$.

Usually, nodes of a network have different degrees. By counting the number of nodes with the same degree $d$ in a graph, one obtains its degree distribution $P(d)$. In the case of directed networks, there are the indegree, $P_{\text {in }}(d)$, and the outdegree, $P_{\text {out }}(d)$, distributions. Additionally, we can construct joint distributions $P\left(d_{i n}, d_{\text {out }}\right)$ which indicate the numbers of nodes with $d_{\text {in }}$ input and $d_{\text {out }}$ output connections in a network. For example, for random Erdös and Rényi networks these distributions are binomial. In regular networks, degrees of all nodes are equal and thus the distributions are singular.

An important further property is the mean degree $D$ that yields the average number of neighbors of each node in the network. If networks are directed, we have $D=D_{\text {in }}+D_{\text {out }}$, where $D_{\text {in }}$ and $D_{\text {out }}$ are the mean input and output degrees, respectively. Because each outgoing connection is at the same time an incoming connection of a different node, we have $D_{\text {in }}=D_{\text {out }}$. The mean degree $D$ can thus be calculated as the number of connections per node in a network.

\section{Clustering coefficient}

This local property of networks is a measure of the density of interactions among a set of nodes. In an undirected network, a node $i$ with $k$ neighbors has clustering coefficient $C_{i}$ defined as the ratio between the actual number of connections $E_{i}$ among its $k$ neighbors and their maximum possible number of connections $k(k-1) / 2$,

$$
C_{i}=\frac{2 E_{i}}{k(k-1)}
$$

For a network, the clustering coefficient $C$ is the average of the clustering coefficients $C_{i}$ of its nodes.

Most of real networks have directed connections and, therefore, one cannot use the definition (1.11) to determine this property. In structural investigations of directed networks, such as in the study of the WWW by Adamic and Huberman [44], networks are often made bidirectional by discarding information about their directions. Although with this solution we can use the definition (1.11), the networks loose one of their main characteristics, their directionality. Moreover, many different directed networks will then be transformed to the same undirected one. 
In our study, we calculate the clustering coefficient of a network by considering that two nodes are neighbors if there exists at least one directed connection between them. In this definition, the clustering coefficient $C_{i}$ of a node $i$ with $k$ neighbors is the ratio between the number $E_{i}$ of directed connections among its $k$ neighbours, and their maximum number of connections $k(k-1)$,

$$
C_{i}=\frac{E_{i}}{k(k-1)}
$$

The advantage of this definition is that it gives the same result as equation (1.11) for undirected networks (considering an undirected connection as two bidirectional ones). However, we do not need then to make any changes in the network and the directionality of its connections is retained. Figure 1.7 shows examples where the clustering coefficients

\begin{tabular}{|l|c|c|c|}
\hline Network & & - & - \\
\hline $\begin{array}{l}\text { Classic clustering } \\
\text { coefficient }\end{array}$ & - & - & $C=1$ \\
\hline $\begin{array}{l}\text { Classic clustering } \\
\text { coefficient (undi- } \\
\text { rected links) }\end{array}$ & $C=1$ & $C=1$ & $C=1$ \\
\hline $\begin{array}{l}\text { Our clustering co- } \\
\text { efficient }\end{array}$ & $C=0.5$ & $C=0.67$ & $C=1$ \\
\hline
\end{tabular}

Figure 1.7: Examples of clustering coefficients of three directed graphs, using the clasical definition (1.11) and the definition (1.12.)

of three directed graphs are calculated by using both definitions.

\section{Paths}

A path in a network is the set of connections that link two nodes. Its length is the number of connections that are in the path. The distance between two nodes is the length of the shortest path among all available ones. When two nodes cannot be connected, the distance between them is taken as infinite. In undirected graphs, the distance between two nodes is independent of the directions in which we move. If such a network is connected (without isolated nodes), we can reach any node of the network starting from any other one.

In directed networks, the situation is similar. However, now we have to consider the directions of the connections. A path between two nodes must connect them following the directions of its links. In consequence, two nodes can be connected only in one direction, or the distances between them can be different, depending on the node from which we start to move. 
Two properties related to the length of these paths are useful to characterize the networks: the diameter and the average path length. The diameter $L$ of a network is the largest distance between any pair of nodes. The average path length $(A P L)$ is the average of the distances between all pairs of nodes in the network. Since the possibility that some pairs of nodes are disconnected is important in directed networks, the diameter and the average path length can be infinite with the previous definitions. However, this can be avoided if we consider only the distances of the paths which really exist in a network.

In systems, which we have considered previously, we find normally the existence of input and output regions and directed connections. In such networks, it is possible to define the diameter $\left(L_{i o}\right)$ and the average path length $\left(A P L_{i o}\right)$ considering only the paths which exist between such two regions. These quantities indicate the number of steps needed by an input signal to be processed and reach some output node. Another important related quantity is the number of connected pairs of input and output nodes which exist in the network, since it measures how many output nodes are reached by the activation of an input node.

\subsubsection{Motif distributions}

The statistical properties, which we have described, give general characteristics of networks. Analyzing real networks, it has been shown [45] that they can have similar degree distributions, short path lengths between the nodes and high clustering coefficients, and nonetheless be very different with respect to their local structures. This suggests that such statistical properites are not enough to characterize the network architecture. Milo et. al. $[46,47,48]$ have proposed to investigate local structures by considering the relative frequency of appearance of small subgraphs, or motifs, as compared to the randomized versions of the same networks.

In the case of directed subgraphs of size three, there are 13 different possible patterns of connections or motifs, which are all shown in Fig. 1.8. Although some of them have the same structural properties, such as the clustering coefficient, they are very different from the point of view of dynamics. For example, motifs 7 and 8, representing the feedforward and the feedback loops, have the same clustering coefficient $C=0.5$. However, only dynamical feedback loops can play a role in the robust adaptation of signal transduction networks to the variation of biochemical parameters [35]. Thus, analyzing the appearance of these motifs in a network allows us to obtain a more detailed structural characterization. Because we want to study the local structure of relatively small networks, we will consider only the motifs with three nodes.

Each of these motifs has a relative appearance $Z_{i}$ in a network $G$, telling whether it is expressed more $\left(Z_{i}>0\right)$ or $\left(Z_{i}<0\right)$ less frequently than in the randomized versions of the same network $G$. After normalization, the relative appearances $Z_{i}$ form a vector $\vec{Z}$, the normalized $Z$ score, which gives us the motif distribution of a given network.

The normalized $\mathrm{Z}$ score is determined as following. The statistical significance of a 

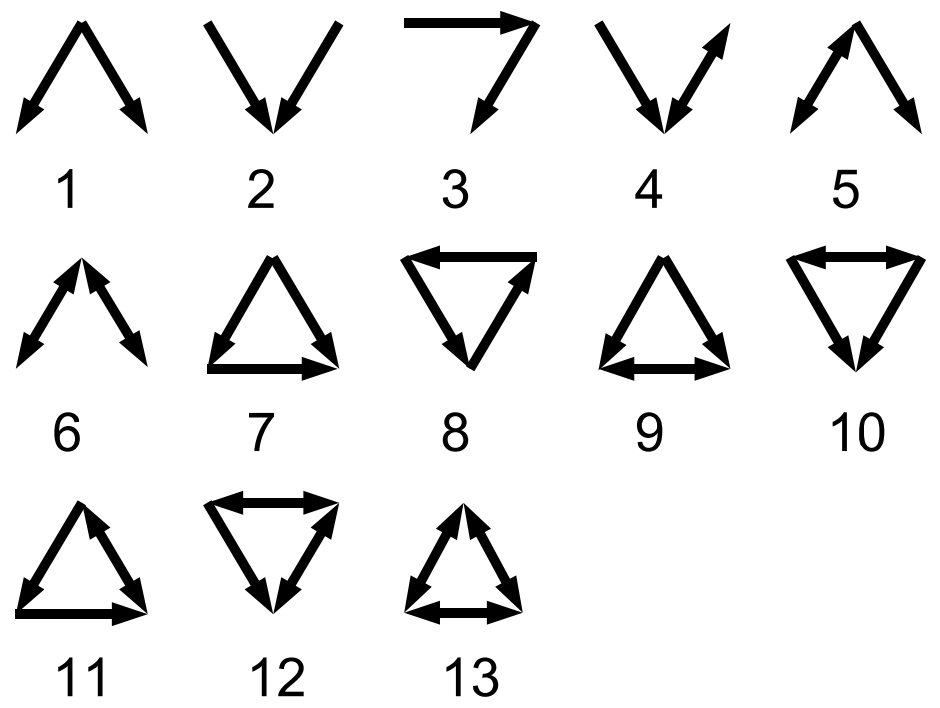

Figure 1.8: All possible three-node motifs. Nodes are the vertices where the links start and to which they arrive.

motif $i$ in a network $G$ is given by its $z_{i}$ score:

$$
z_{i}=\frac{N_{i}^{\text {real }}-\left\langle N_{i}^{\text {rand }}\right\rangle}{\operatorname{std}\left(N_{i}^{\text {rand }}\right)}
$$

where $N_{i}^{r e a l}$ is the number of times that the subgraph $i$ appears in the network $G$, and $\left\langle N_{i}^{\text {rand }}\right\rangle$ and $\operatorname{std}\left(N_{i}^{\text {rand }}\right)$ are the mean and the standard deviation of its appearances in the ensemble of randomized networks. Such randomized networks have the same degree distributions and the degree sequence (i. e. the number of nodes with a specific input and output degrees) as the analyzed network $G$, but they represent new random patterns of connections.

The normalized $Z$ score is given by a vector with the components

$$
Z_{i}=\frac{z_{i}}{\sqrt{\sum_{k=1}^{13} z_{k}^{2}}} .
$$

With this normalization, the relative significance of a subgraph is more important than its absolute significance. This is useful in the comparison of networks with different sizes, since the number of appearances normally increases with the size of the networks.

\subsection{Network superfamilies}

Analyzing three-node motif distributions of many networks of different fields and origins, Milo et. al. [46] have found that there are remarkables similarities between such 


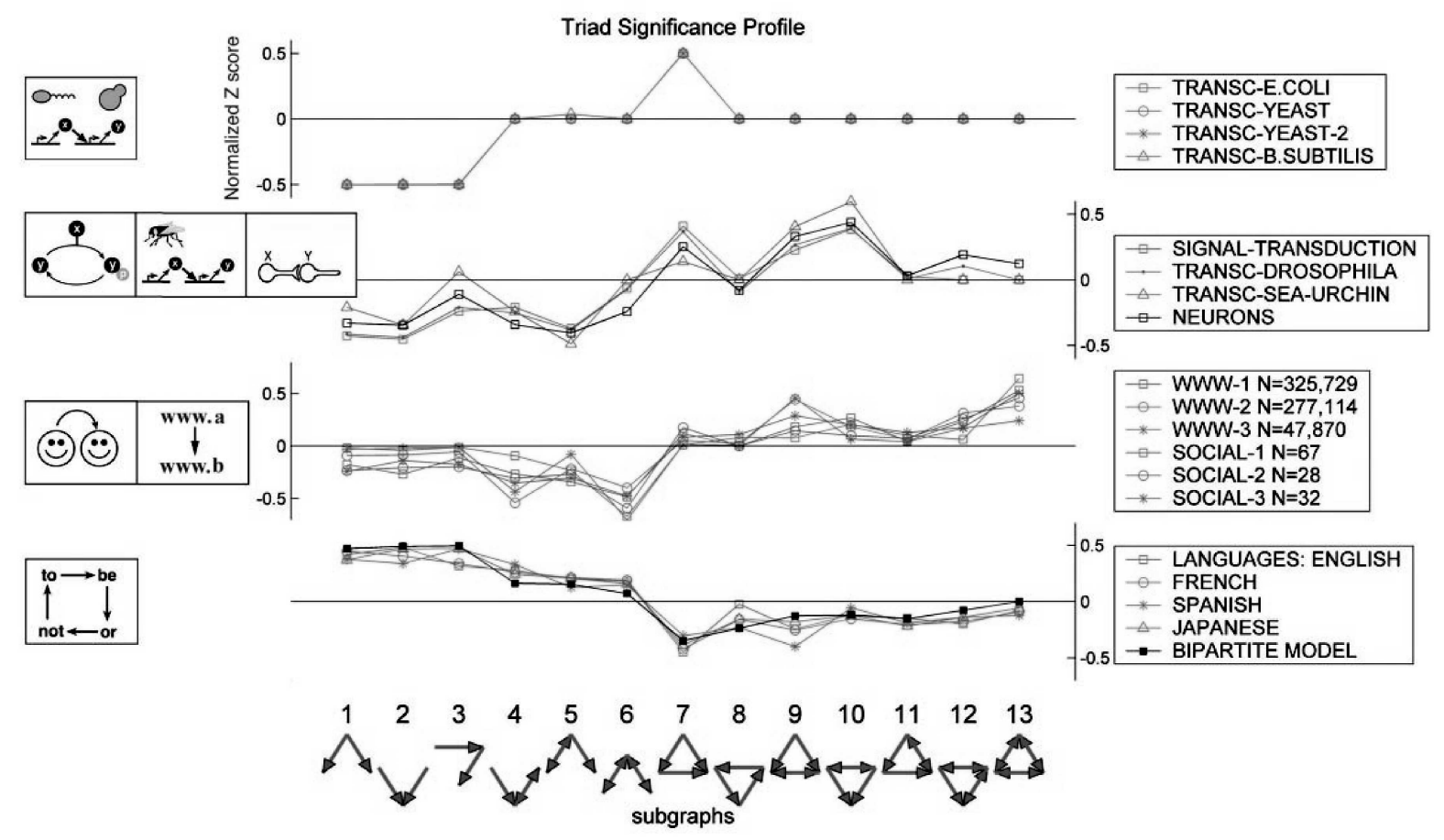

Figure 1.9: Three node motif profiles of networks from various disciplines. Networks with similar characteristic distributions are grouped into superfamilies (from Milo et. al. [46]).

distributions and it is possible to identify four main superfamilies. Figure 1.9 shows different motif distributions for each superfamily.

In the first superfamily, several sensory transcription networks that control gene expression in bacteria and yeast in response to external stimuli are found. In Fig. 1.9 motif distributions of three microorganisms, the bacteria Escherichia coli and Bacillus subtilis and the yeast Saccharomyces cervisiae, are given. Such motif distributions show increases in the appearance of motif 7, the "feedforward loop". This loop is related to signal-processing tasks, such as persistence detection, pulse generation, and acceleration of transcription responses. The motif 3, the 3-chain, is underrepresented, because of the shallow architecture of these networks which have only few long cascades. These networks are "sensory networks" which need to respond within minutes to transient signals such as stresses and nutrients. The minimal time required for a response (for the first proteins to be expressed) is indeed on the order of minutes. If the information needs to pass additional steps (a regulator protein needs to be expressed and cross its activation threshold to turn on a gene), then the response time becomes much longer. Thus, these networks can be understood as "rate-limited networks", where the desired response times are often as short as the response times of the network components [46]. In the rate-limited network superfamily, long cascades and feedback loops are rare.

The second superfamily includes three kinds of information-processing networks in 
biological macroorganisms: signal-transduction in mammalian cells, development transcription networks that guide the development in fruit fly and sea-urchin, and synaptic connections between neurons in Caenorhadbitis elegans. In these networks, motifs 7, 9 and 10 are enhanced, whereas by motifs $1,2,4$ and 5 are suppressed. In contrast to the networks of the previous superfamily, these networks include two-node feedbacks that regulate or are regulated by a third node (motif 10 and 9) and are less biased against cascades (motif 3 ). The common feature to this superfamily of information-processing networks is that the response time of each step in the network is usually much shorter than the response time required for the biological function of the network. For example, protein signal-transduction networks often need to respond within an hour or longer, but each interaction can take minutes or less. Cascade steps in developmental networks can have response times of tens of minutes, but the processes they control are much slower, on the order of animal cell-division times that can take several hours. It has been therefore suggested [46] that this superfamily characterizes biological information-processing networks which are not rate-limited

Several WWW networks of hyperlinks between Web pages and some social networks show similar three node motif distributions and they are combined in the third superfamily. In this superfamily, motifs 9, 10, 12 and 13, that represent triangle transitive interactions are overrepresented, whereas motifs 4,5 and 6 have low frequencies.

Finally, the last superfamily is formed by networks of word-adjacency. These networks are constructed by taking as nodes the words of a text. A directed connection between two nodes exists if these two words appear consecutively in the text. In Fig. 1.9 motif distributions of several languages (English, Frensh, Spanish, and Japanese) taken from several texts are shown. The main characteristic of these motif profiles is that motifs from 7 to 13 are underrepresented. According to Ref. [46], the lower significances of the motifs with triangles can be explained by the structures of the languages, where words have different categories and a word from a certain category tends to be followed by one from a different category (for example, a preposition is usually followed by nouns or articles ). The bipartite networks (also shown in Fig. 1.9), where there are two classes of nodes and the connections are allowed only between nodes of different class, yield a similar motif distribution. 


\section{Chapter 2}

\section{Flow distribution networks}

In this chapter we present a model of flow distribution networks based on the characteristic features of such biological systems as signal transduction, genetic expression and neural networks. The model is a gross simplification of the real systems. However, it retains their principal structure with the presence of input, output and processing regions. In the model, nodes carry out the processing function, while the input-output relationship defines the task of a network. The operation of a network in this model strongly depends on its architecture. We introduce the flow error, a measure of the performance of a network with respect to a prescribed input-output pattern.

\subsection{The pipeline network model}

Biological systems, discussed in the previous chapter, have common functions. They process input signals and express the results by activating output elements which produce output signals. In signal transduction networks inside the cells, input signals are external stimuli applied to specific receptors which activate particular pathways and, finally, lead to the production of proteins with certain biological effects. In a similar way, genetic networks have as input signals the activation of certain genes whose expressions produce, afterwards, the activation of other genes. The sets of genes activated through such processes form the output signals of the networks. Finally, in neural systems, input neurons are activated by external stimuli. They send signals to groups of neurons, which process this information, and the results are expressed by the output neurons.

As we have seen in the previous chapter, detailed mathematical models are available for such biological networks. However, we do not want to choose one of these models for our investigations. First of all, we would like to obtain the results which are general and do not strongly depend on the details of a particular realistic model. Moreover, our investigations involving design of networks through a computer evolution process would have been too computationally intensive if such real network models were chosen.

In our investigations, a simple model of pipeline networks will be used. In such net- 
works, directed connections can be viewed as pipelines that transport material fluxes between the nodes. The nodes operate like redistribution centers which split the total incoming flux among the outgoing pipes. With these elements, we can construct networks with the architectures similar to those of real systems and with the three regions: the input region with the source nodes, the middle region which distributes the input fluxes (processing), and the output region with the nodes that pump the resulting fluxes out of the network. An example of a pipeine network is shown in Fig. 2.1.

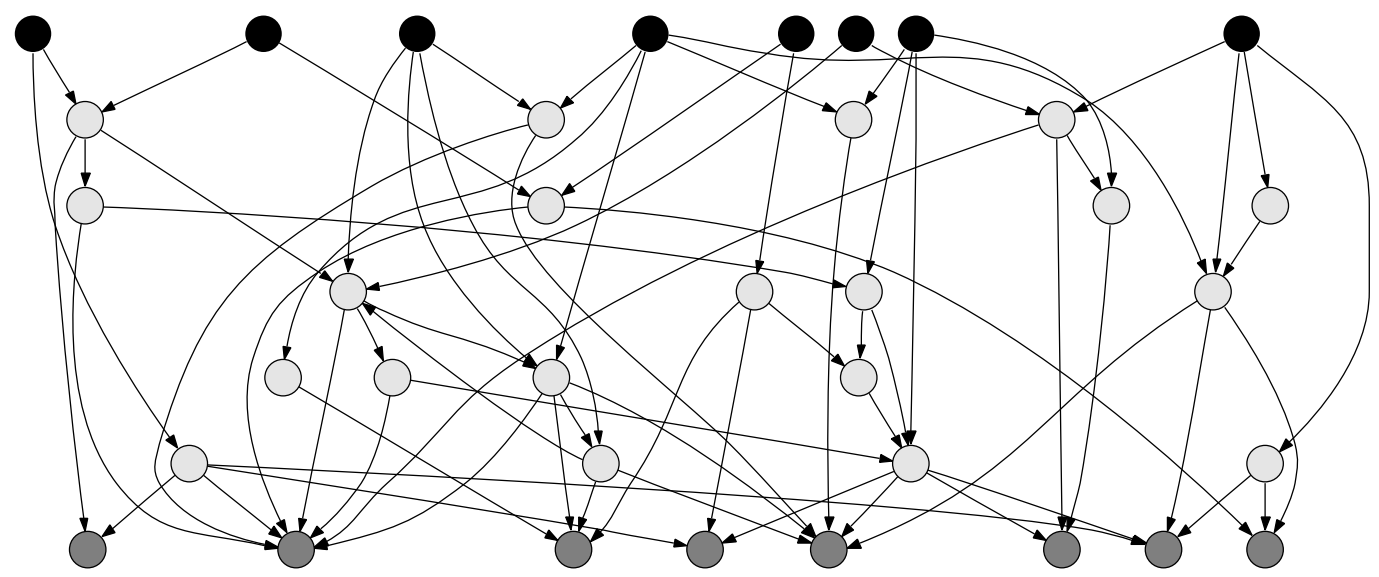

Figure 2.1: Example of a flow distribution network. Input nodes are black, middle nodes are light gray, and output nodes are dark grey.

The model is static. The fluxes are stationary and, thus, the considered signals have no temporal dependence. This is a strong simplification, since temporal patterns of signals are important in real systems. However, it allows us to focus exclusively on the architecture of the networks disregarding any dynamical phenomena. Additionally, with this simplification, the computational demand decreases significantly and we can perform large simulations and study statistical properties of such networks.

The nodes operate in a simple way. They integrate input fluxes and divide the total incoming flux in equal parts among the outgoing connections. They only redistribute the flow and cannot accumulate input fluxes. This operation mode is linear and involves no thresholds or particular activation function.

Below, a detailed description of the considered pipeline network model is provided.

\subsubsection{Network topology}

A network $G$ has $N$ nodes divided into three kinds, according to their functions. There are $N_{\text {in }}$ input nodes, $M$ middle nodes and $N_{\text {out }}$ output nodes. Two nodes can be linked by a directed connection. Not all connections in the network are allowed. The following connection rules restrict interactions between the nodes: 
1. An input node can be connected only with the middle nodes; the connections go from an input node to the middle nodes.

2. A middle node can be connected with other middle nodes and with the output nodes. In the second case, the connection must go from a middle node to an output node.

3. An output node can only have input connections and such connections must come from the middle nodes.

4. It is not allowed to have multiple connections between two nodes (with the same direction) and loops of length one (connections from a node to itself).

The topology of a network $G$ can be described as a directed graph with an adjacency matrix $\mathbb{A}$, whose elements are $\mathbb{A}_{j i}=1$, if there exists a connection from the node $i$ to node $j$, and $\mathbb{A}_{j i}=0$ otherwise.

\subsubsection{Network operation}

The networks operate through the fluxes established on the connections, when external fluxes activate the input nodes. A node integrates the fluxes coming from its input connections and it splits such total flux among its outgoing connections, in equal quantities. The total input flux $x_{i}$ at a node $i$ is the sum of all input fluxes $u_{i j}$ coming from the nodes $j$ to the node $i$ plus a possible external flux $u_{i}^{e x t}$ :

$$
x_{i}=\sum_{j=1}^{I(i)} u_{i j}+u_{i}^{e x t}
$$

where the sum is taken over all incoming connections to the node $i$, and $I(i)$ is the incoming degree of the node $i$ (the total number of such connections).

A flux $u_{l k}$ along a connection from the node $k$ to the node $l$ has an intensity given by the total input flux of node $x_{k}$ divided by the total number of outgoing connections $O(k)$ of this node:

$$
u_{l k}=\frac{x_{k}}{O(k)}
$$

Combining equations (2.1) and (2.2), and extending the sum over the whole network, we have

$$
x_{i}=\sum_{j=1}^{N} A_{i j} \frac{x_{j}}{O(j)}+u_{i}^{e x t} .
$$

This algebraic system of linear equations gives the total input flux of each node of the network when external fluxes are applied through the input nodes. The operation of a network is static with the fluxes established simultaneously on the whole network when the inputs are changed. 
Fig. 2.2 shows an example of the operation of a simple network. External unit fluxes are applied to the input nodes and are redistributed several times by the middle nodes. Finally, fluxes with different intensities arrive to the output nodes. Since there is no accumulation or loss of material in the nodes, the entire flux applied to the input nodes reaches the output nodes. Therefore, each node in the network receiving some flux must

a

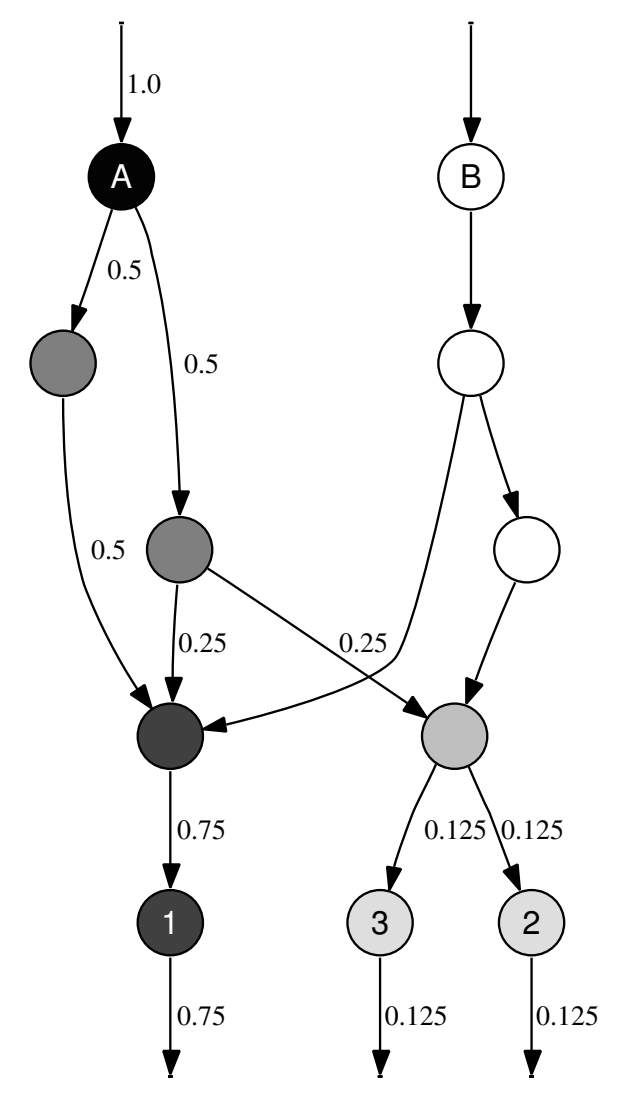

$\mathrm{b}$

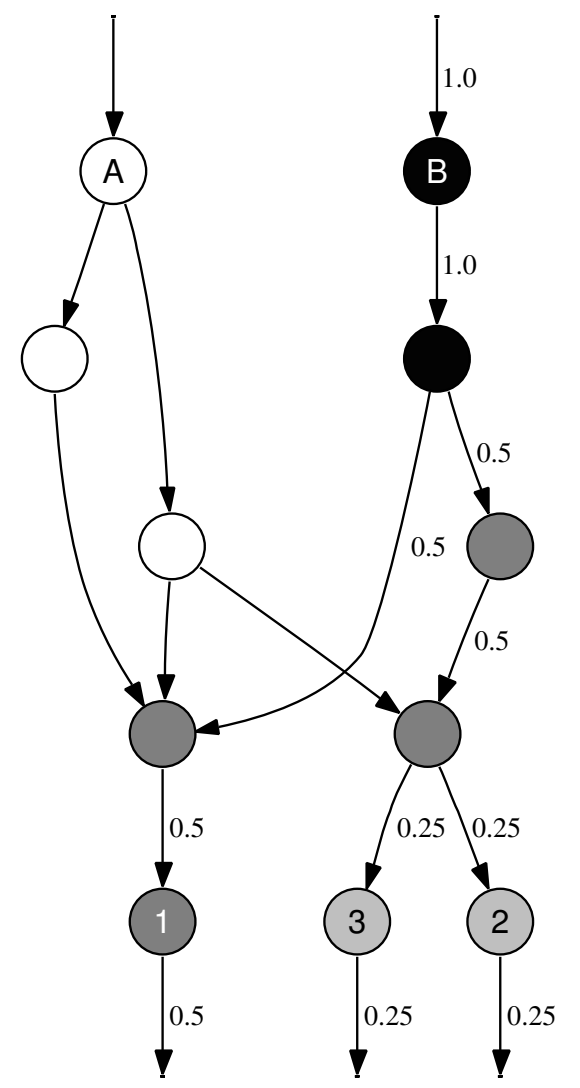

Figure 2.2: Example of a network with $N=11$ nodes, $N_{i n}=2, M=6$ and $N_{\text {out }}=3$. (a) Fluxes in the connections when the input node $A$ is activated by an unit external flux. (b) Fluxes when the input node B is activated. The total input flux in each node is displayed by grey scale, varying from black, when it is one, to white, when it is zero.

be effectively connected to at least one output node through a certain path.

The conservation of the total input flux implies that

$$
\sum_{i=1}^{N_{\text {in }}} u_{i}^{e x t}=\sum_{j=1}^{N_{\text {out }}} x_{j}
$$

i. e. the total external flux applied to the input nodes is equal to the total fluxes arriving to the output nodes. Although such networks have definite nodes with the output function, 
middle nodes without outgoing connections must also be considered as output nodes and taken into account in (2.4).

\subsubsection{Algebraic aspects}

The system (2.3) of linear algebraic equations can be determinate, indeterminate or incompatible, depending on the architecture of the network. Fig. 2.3 shows three networks with different structures and their systems of equations when they are activated by unit fluxes. Fig. 2.3a corresponds to a network that has a determinate system of equations and shows its solution. Most of the networks we study in our work have this kind of mathematical structure.

Figure 2.3b displays a network that leads to an indeterminate system of equations. An infinite number of solutions, with fluxes $x_{4}$ and $x_{5}$ being equal, but otherwise arbitrary, is possible. Disconnected loops do not affect operation of the network and we put these respective fluxes equal to zero.

a

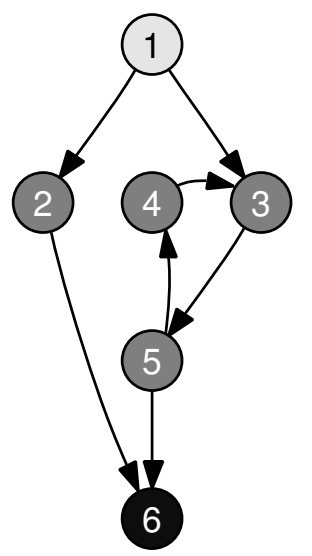

$x_{1}=1$

$x_{2}=0.5 x_{1}=0.5$

$x_{3}=0.5 x_{1}+x_{4}=1$

$x_{4}=0.5 x_{5}=0.5$

$x_{5}=x_{3}=1$

$x_{6}=x_{2}+0.5 x_{5}=1$ $\mathrm{b}$

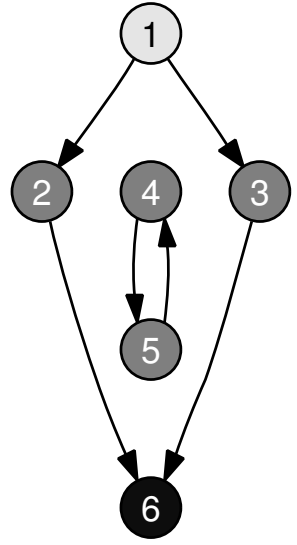

$x_{1}=1$

$x_{2}=0.5 x_{1}=0.5$

$x_{3}=0.5 x_{1}=0.5$

$x_{4}=x_{5}=0$;

$x_{5}=x_{4}=0$;

$x_{6}=x_{2}+x_{3}=1$
C

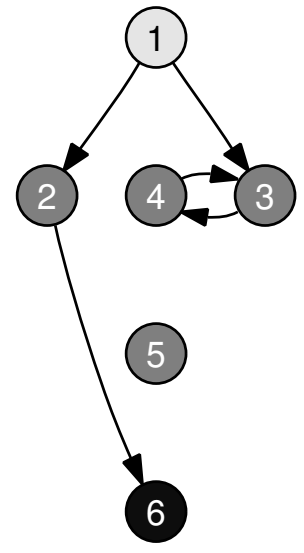

$x_{1}=1$

$x_{2}=0.5 x_{1}=0.5$

$x_{3}=0.5 x_{1}+x_{4}$

$x_{4}=x_{3}$

$x_{5}=0$

$x_{6}=x_{2}=0.5$

Figure 2.3: Simple networks of $N=6$ with $N_{\text {in }}=1, M=4$ and $N_{\text {out }}=1$. The algebraic system of linear equations can be determinate (a), indeterminate (b), and incompatible (c).

Finally, Fig. 2.3c presents a network with an incompatible (contradictory) system of linear equations. In this case, there are no solutions for the input fluxes in nodes 3 and 
4. Such networks are not functional, because accumulation of flow in the nodes is not possible and the fluxes which arrive to these two nodes cannot be extracted from them. Incompatible systems have subgraphs representing loops that have no connections to the output nodes. A network with this property may emerge from a functional network after a mutation during the evolutionary optimization process. Moreover, after another mutation it can give rise again to a functional network. Therefore, we do not want to discard such incompatible networks during the evolution process (although they are never accepted as final solutions). To eliminate the incompatibility, fluxes going to the loops are removed through the addition of new virtual output nodes connected to them. In Fig. 2.3c with this assumption, the flux going from node 1 to node 3 is lost and the solution becomes possible.

\subsection{Input-output relationships}

The relationship between the input and output fluxes yields the function of a network. Since our networks operate linearly, it is enough to analyze the response of a network when only one input node is activated by applying an unit flux to it.

The output pattern is the relationship between the input and output fluxes, which is described by a matrix $\mathbb{Q}$. An element $\mathbb{Q}_{j \alpha}$ of this matrix represents the flux in the output node $j$, when the input node $\alpha$ is activated by an unit flux. Fig. 2.4 shows a typical network and its output pattern matrix. There, the output matrix $\mathbb{Q}$ is displayed in grey scale. This scale varies from white, when there is no arriving flux, to black, when the maximum flux unity is reached.

a

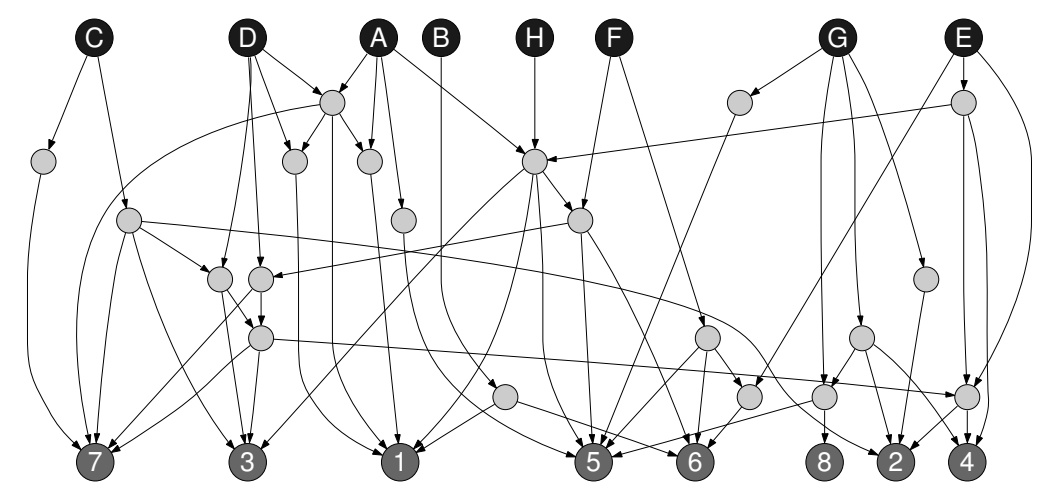

b

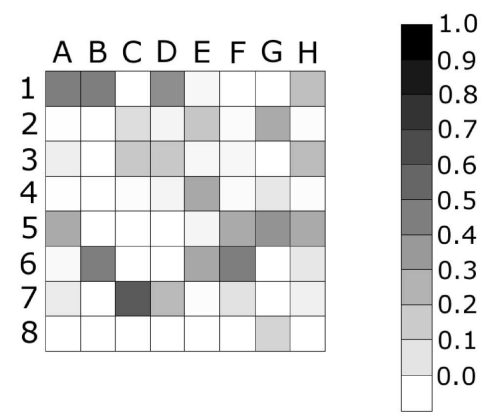

Figure 2.4: (a) Network of $N=36$ with $N_{\text {in }}=8, M=20$ and $N_{\text {out }}=8$. (b) Its output pattern matrix $\mathbb{Q}$ displayed in grey scale.

Although some middle nodes can act like the output nodes, we do not consider them here, because we are interested only in the activation of the nodes in the output region. The output patterns $\mathbb{Q}$ depend only on the architecture of a network. One of our main 
objectives is to design networks corresponding to particular prescribed input-output patterns.

\section{Target output patterns}

The structures of biological networks are not accidental, because they should generate well-defined output patterns. In neural networks, only some output neurons should be activated when a particular stimulus activates the receptors. In signal transduction networks, not all possible output proteins are produced in response to an initial stimulus, but only the proteins which belong to the respective activated pathway. Similarly, in genetic networks only a set of genes becomes activated in response to a certain input. Additionally, in such systems, when many output elements are activated as response to a stimulus, they usually have different individual responses. For example, different concentrations are possible for the activated output proteins of a signal transduction network and, in a neural network, activated neurons can have different firing frequencies.

Our networks will be designed to produce prescribed output patterns resembling those of the real networks. In the target output patterns, which we will use, activation of an input node produces the activation of only a subset of output nodes. The nodes, which will be activated, and their activity levels are randomly chosen.

Explicitly, the following procedure is used to generate target output patterns: we define a parameter $K$, which gives the total number of output nodes activated by an input node. Thus, each column of the output pattern matrix $\mathbb{Q}^{0}$ has $K<N_{\text {out }}$ elements different from zero. The positions of these $K$ elements in the column are chosen randomly. In addition, since the total flux must be unity, we choose random values $Q_{i \alpha}$ and then normalize each column $\alpha$ to unity by taking:

$$
\mathbb{Q}_{j \alpha}^{0}=\frac{Q_{j \alpha}^{0}}{\sum_{k=1}^{N_{o u t}} Q_{k \alpha}^{0}} .
$$

Values $\mathbb{Q}_{j \alpha}^{0}$ give the activities of the output nodes in the target output pattern when an input node $\alpha$ is activated.

\section{Flow error}

There may be a difference between the actual output pattern $\mathbb{Q}$ of a network and its prescribed target output pattern $\mathbb{Q}^{0}$. We can measure this difference by introducing the flow error $\epsilon$ of the network $G$ :

$$
\epsilon(G)=\frac{1}{2 N_{\text {in }}} \sum_{\alpha=1}^{N_{\text {in }}} \sum_{j=1}^{N_{\text {out }}}\left(\mathbb{Q}_{j \alpha}^{0}-\mathbb{Q}_{j \alpha}\right)^{2}
$$

With this definition, the flow error can vary between zero and one. We do not normalize 
the error $\epsilon$ dividing it by the total number $N_{\text {out }}$ of the output nodes, because our target output patterns always have only $K$ nodes activated and $K$ may be much smaller than $N_{\text {out }}$ in the larger networks. 


\section{Chapter 3}

\section{Evolutionary design of flow distribution networks with prescribed output patterns}

This chapter is divided into two parts. First, we formulate a method to design networks with prescribed functions defined by the target output patterns. We show that the design can be carried out using an evolutionary optimization process, by applying structural mutations to the networks and selecting them according to their new performances. We analyze such aspects of the evolutionary process as the schemes of mutations and the convergence of the used algorithm depending on its parameters.

The second part of this chapter is devoted to a study of the characteristics of the networks obtained by using this evolutionary process. We consider how architectures of the networks depend on their sizes, on the characteristics of the prescribed output patterns and on the schemes of mutations used.

\subsection{Evolutionary design method}

The number of possible connections in a network of size $N$ with $M$ middle nodes, consistent with the connection rules is $M(N-1)$. Therefore, there are $2^{M(N-1)}$ possible networks because any element of the adjacency matrix can be either 0 or 1 . For example, for $N=36$ and $M=20$, the total number of possible networks is, therefore, of the order of $2^{700}$. This number is astronomically large (more than the total number of atoms in the Universe). Therefore, one cannot just try all networks one after another until a network with the best performance with respect to the chosen output pattern is found. On the other hand, no rational methods are known which would allow to determine the architecture of a network generating an arbitrary prescribed target pattern.

In our study, an evolutionary optimization method is used. It is similar to the Metropo- 
lis algorithm known in statistical mechanics. An analogous optimization scheme was earlier applied [51] to reconstruct networks from their Laplacian spectra. The algorithm is based on the minimization of a cost function, the flow error in our case, through mutations and subsequent selections. The mutations we use are specifically designed to rapidly minimize the error and do not have a biological counterpart. Two different schemes of evolutionary mutations will be employed.

\subsubsection{Optimization algorithm}

Starting from an initial network $G_{0}$, having some flow error $\epsilon_{0}$ with respect to the prescribed output pattern $\mathbb{Q}^{0}$, the flow error is gradually minimized using an iterative process of structural mutations and selections.

In each iteration, we apply a mutation to the current network $G$ with the flow error $\epsilon$ and obtain a new network $G^{\prime}$ with the flow error $\epsilon^{\prime}$. If $\Delta \epsilon=\epsilon^{\prime}-\epsilon<0$, we accept the mutation and replace $G$ by $G^{\prime}$. If $\Delta \epsilon>0$, we accept the mutation replacing $G$ by $G^{\prime}$ with the probability $\exp (-\Delta \epsilon / \sigma \epsilon)$. We repeat iterations, until the flow error becomes smaller than a certain threshold value or for a fixed number of iterations.

The initial network $G_{0}$ is constructed as a random Erdös-Rény network of size $N$ and the connection probability $p$, taking into account the connection rules of the pipeline model. The parameter $p$ is chosen as a random number in the interval between 0 and 1 in each evolution. The initial networks usually have large flow errors $\epsilon_{0}$.

The effective temperature in this Metropolis algorithm is $T=\sigma \epsilon$ and, therefore, it decreases with the flow error. Optimization algorithms, where the temperature decreases together with the property which is minimized, are known as the methods of "simulated annealing" [49].

During the evolutions, some networks can have isolated middle nodes. These nodes have no function in the operation of the networks. However, they can be useful in the subsequent networks, after the application of an evolutionary mutation. Therefore, we keep fixed the number of middle nodes during the evolution, even when they have no function. Such nodes are removed from the networks at the final stage, when the evolution is finished.

\subsubsection{Evolutionary mutations}

We have tried several different mutation schemes used to construct the networks. Below we present two schemes with good performances. In the first scheme, a mutation modifies a link and produces a small local change. In the second scheme, mutations produce a larger change in the network architecture through the addition or deletion of new paths between input and output nodes. 


\section{Link mutations}

In this mutation scheme, we add a new connection to the network or delete an existing one from it, with the same probability. The effect of such a mutation on the architecture of the network is local and rather weak, because it affects only a connection between two nodes.

\section{Path mutations}

In this scheme, a mutation consists of adding to the network or deleting from it (with the same probability) a certain random subgraph $g$. This subgraph is a path of length $L$ leading from an input node $i$ to an output node $j$, which crosses the network through $L-1$ middle nodes. The nodes $i$ and $j$, the middle nodes and the length $L$ are all randomly chosen. A middle node can be repeated in $g$, but we require that it does not appear consecutively, in order to avoid loops of length one ${ }^{1}$. Fig. 3.1 shows an example of such mutation.

$\mathbf{a}$

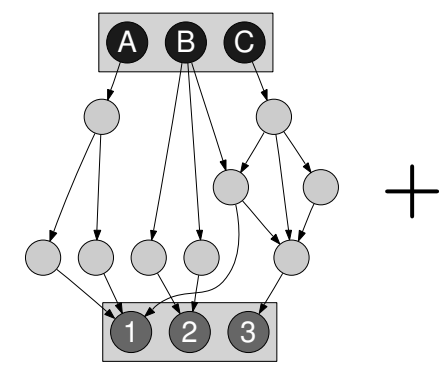

b

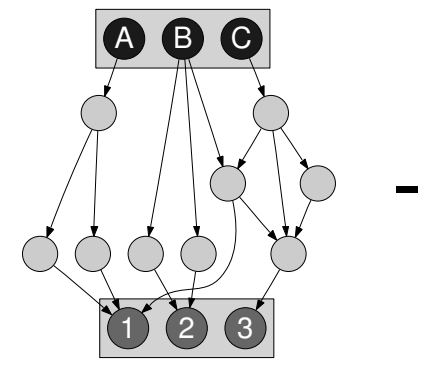

G
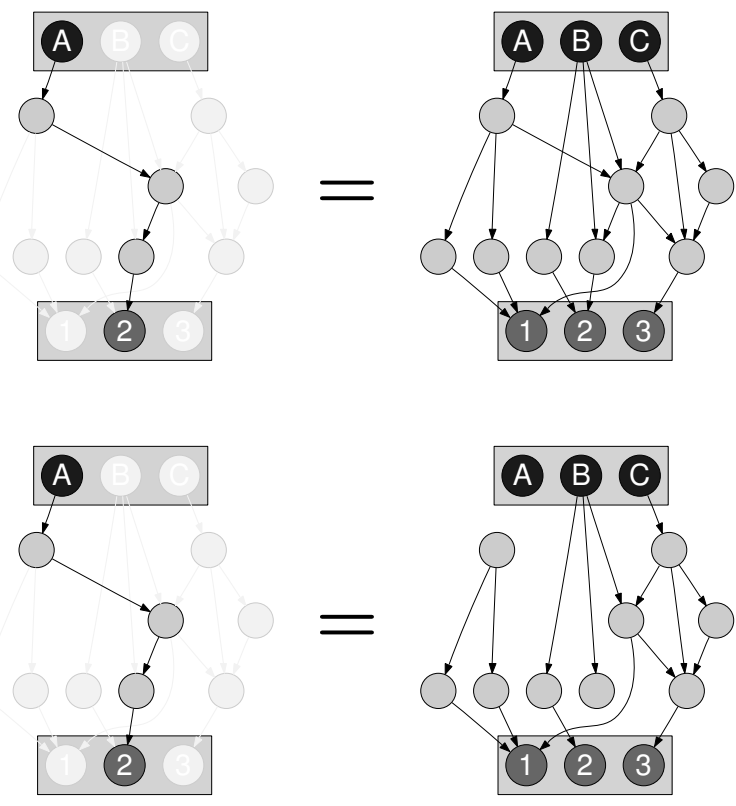

$g$

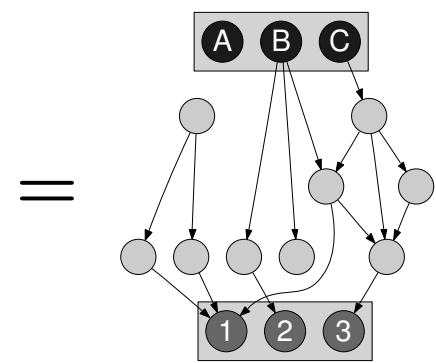

$G^{\prime}$

Figure 3.1: The network $G$ is mutated to network $G^{\prime}$ through the addition (a) or deletion (b) of the path $g$.

\footnotetext{
${ }^{1}$ Formally, in the graph theory a path between two nodes must go through different nodes.
} 


\subsubsection{Example of network construction}

Figure 3.2 shows an example of the construction of a network with a prescribed target output pattern. In Fig. 3.2a, dependence of the flow error $\epsilon$ of the network on the iteration number is displayed. Here, the path mutation scheme is used and the number of the middle nodes $(M=20)$ is fixed during the evolution. The initial network is a random
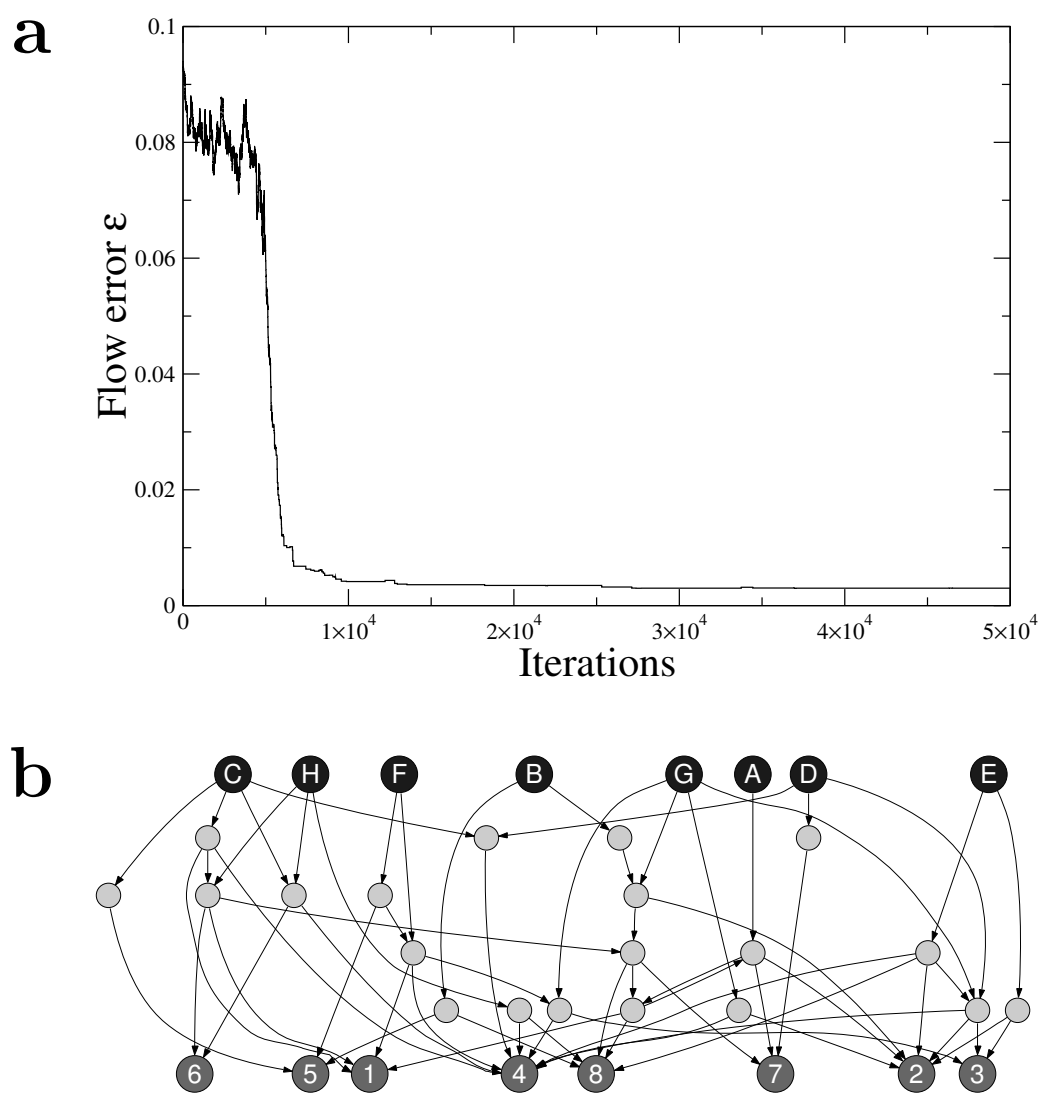

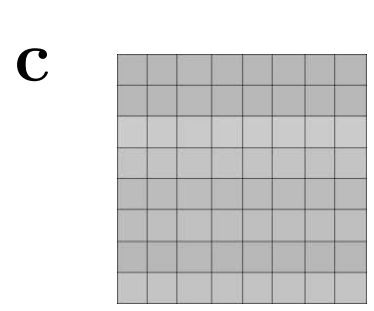

Initial

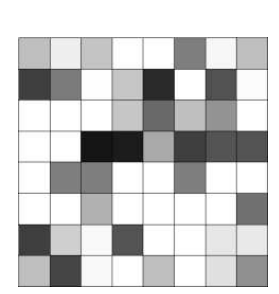

Final

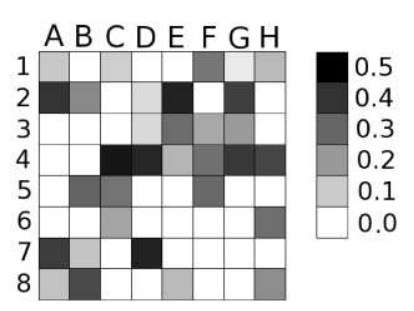

Target

Figure 3.2: Example of a network construction. The temperature parameter is $\sigma=10^{-4}$. The network has $N=36$ nodes with $N_{\text {in }}=8, M=20$ and $N_{\text {out }}=8$; the target output pattern has $K=4$. (a) Temporal evolution of the flow error $\epsilon$. (b) Final network obtained after $5 \times 10^{4}$ iterations. (c) Initial, final and target output patterns displayed in the gray scale.

graph with the restrictions given by the connection rules and, in this case, its connectivity 
is $p=0.5$. Figure $3.2 \mathrm{~b}$ presents the final network obtained in the evolution. In Fig. 3.2c, the initial, final and target patterns are shown.

\subsubsection{Comparison of two mutation schemes}

By running many different evolutions starting with different initial conditions, ensembles of networks, optimized with respect to randomly generated target output patterns with the same number of activated nodes $K$ and with the same parameters $N_{i n}, M$, $N_{\text {out }}$, can be constructed. Since the parameters are the same, statistical properties of the designed networks can be considered. To investigate the difference in the efficiency of two mutation schemes (link and path mutations), we have performed 100 independent evolutions for each of these two schemes.

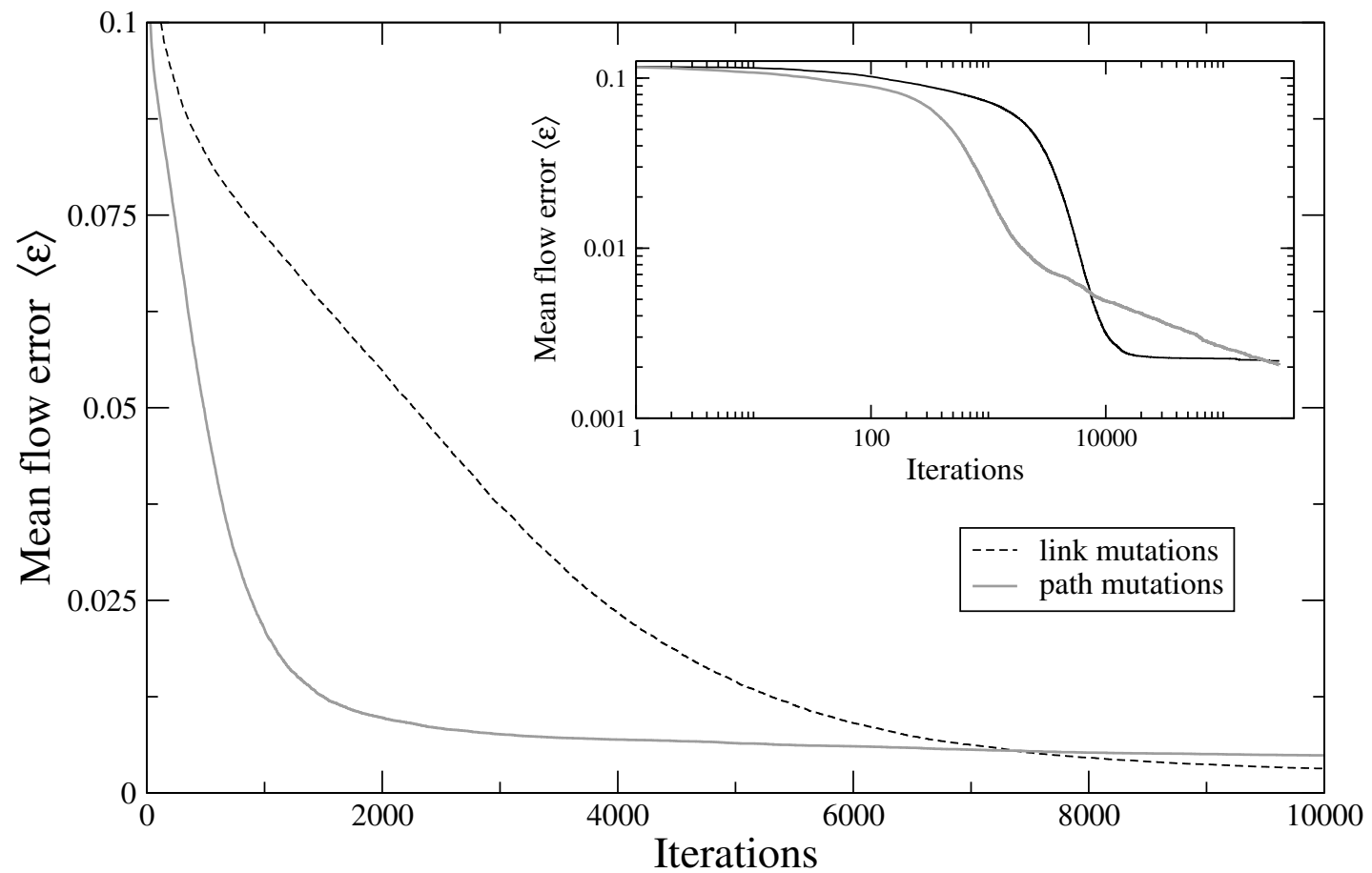

Figure 3.3: Mean flow error $\langle\epsilon\rangle$ of the two ensembles of 100 networks (link and path mutations) as a function of the iteration number. The main plot shows the first stages of the evolution, whereas the inset displays the complete simulation in the $\log -\log$ scale. The parameters are $N_{\text {in }}=8, M=20, N_{\text {out }}=8$ and $\sigma=$ $10^{-4}$. Different initial conditions were used in each evolution. As a target, different randomly generated target output patterns with the same $K=4$ were chosen.

Figure 3.3 shows the mean flow error $\langle\epsilon\rangle$ of the two ensembles as a function of the iteration number. When the path mutation scheme is used, the mean flow error decreases first faster than for the link mutations. The final mean flow error of the two ensembles after $3 \times 10^{5}$ iterations is however practically the same. Thus, the link mutation scheme shows a significantly slower convergence than the path mutation scheme. Our further 
simulations with the networks of larger sizes $N$ have revealed that the difference in the convergence rates increases with the network size $N$.

We can understand these different behaviors if we consider how a network evolves through each kind of mutations. Under path mutations, the probability to correctly connect an input node to an output node $\left(\mathbb{Q}_{i j}^{0} \neq 0\right)$ in a given path is $K / N_{\text {out }}$. This probability is relatively high and it is responsible for the rapid decrease of the flow error in the first iterations. However, once the network has a small flow error any path mutation has a long-range influence. Typically one path can change largely the structure of the network and therefore the convergence rate decreases.

Link mutations operate in a different way. Each link mutation has usually only a small effect on the flow error and such mutations are often accepted. As a result of their accumulation, clusters of modified links are formed, which already have a substantial effect on the output patterns. The development of such clusters and their subsequent evolution involve many individual iterations and, therefore, the evolution is much more slow in this case.

\subsubsection{The temperature parameter}

The temperature parameter $\sigma$ determines the rate of convergence in the optimization process. To study the dependence of the optimization efficiency, evolutions with different values of the parameter $\sigma$ have been performed.

Figure 3.4 shows mean flow errors $\langle\epsilon\rangle$ after $10^{5}$ iterations for different ensembles, each consisting of 100 networks, as a function of the temperature parameter $\sigma$. All networks have $N_{\text {in }}=8, N_{\text {out }}=8$ and their different randomly generated target output patterns have $K=4$, but different ensembles have different numbers of middle nodes $M$. Figure $3.4 \mathrm{a}$ presents the mean flow error $\langle\epsilon\rangle$ of the networks constructed using path mutations, whereas Fig. 3.4b shows similar averages, but using link mutations.

We see that in both cases there is a critical value of $\sigma$ above which convergence is not possible. Below this critical value, the optimization efficiency does not significantly depend on $\sigma$.

The critical values depend on the size of the networks and the mutation schemes used during the evolution. These values decrease when the size of the networks increases. Additionally, in the networks with the same size, but obtained using different mutations, the critical value is smaller when using link mutations.

\subsection{Optimization statistics}

In this section, we investigate how the flow error and the structure of the constructed networks depend on the number of middle nodes, on the characteristics of the target 

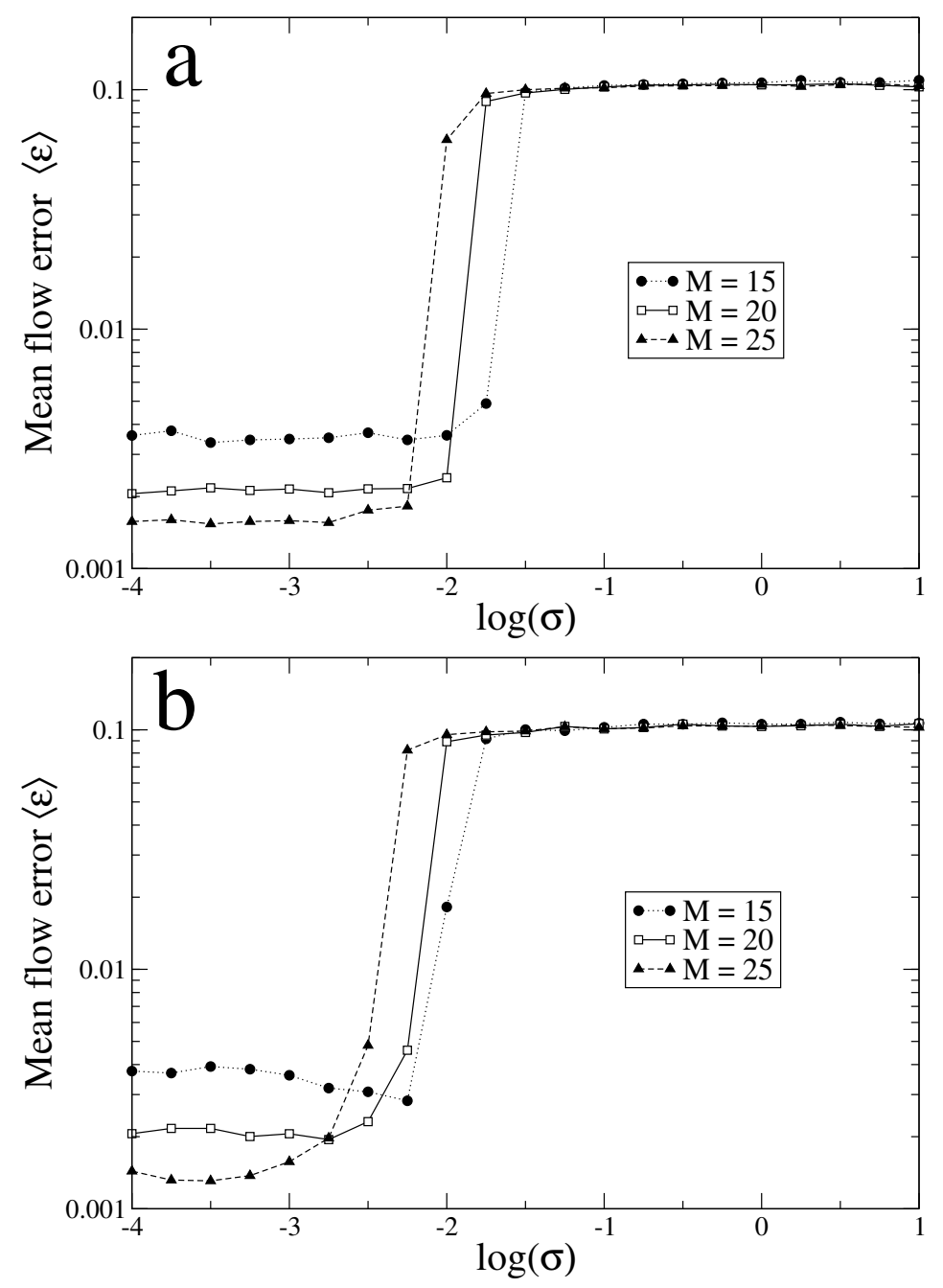

Figure 3.4: Dependence of the mean flow error $\langle\epsilon\rangle$ on the temperature parameter $\sigma$ for ensembles of 100 networks each with $N_{\text {in }}=8, N_{\text {out }}=8, K=4$ and different numbers of middle nodes. Networks constructed (a) by using path mutations and (b) by using link mutations.

output pattern $\mathbb{Q}^{0}$, and on the mutation schemes used during the optimization process. In all simulations in this section, the mean values of different statistical properties, obtained by averaging over ensembles of networks, are presented.

The considered ensembles of networks are obtained by running many independent evolutions. In each evolution, a different randomly generated output pattern (but with the same $K$ ) is used. Each evolution begins with a different randomly generated initial condition. As the initial conditions, Erdös-Rényi networks with randomly chosen connectivities $p$ are taken. The temperature parameters are fixed as $\sigma=10^{-4}$ for both mutation schemes. Each evolution consists of $10^{5}$ iterations. Each of the considered ensembles consists of 100 designed networks for each chosen set of the parameters $M$ and $K$. The numbers of input and output nodes are $N_{\text {in }}=N_{\text {out }}=8$ in all considered networks. 


\subsubsection{Flow error}

Using a network of a given size $N$, it is not generally possible to exactly reproduce an arbitrary prescribed target output pattern. This is because the input flux is split into equal parts in each distribution node and, therefore, only certain rational fractions of this flux can reach the output nodes. The more middle nodes the networks have, the better the output patterns may approximate the target patterns. Therefore, the number of middle nodes is the main parameter determining the minimum remaining value of the flow error.
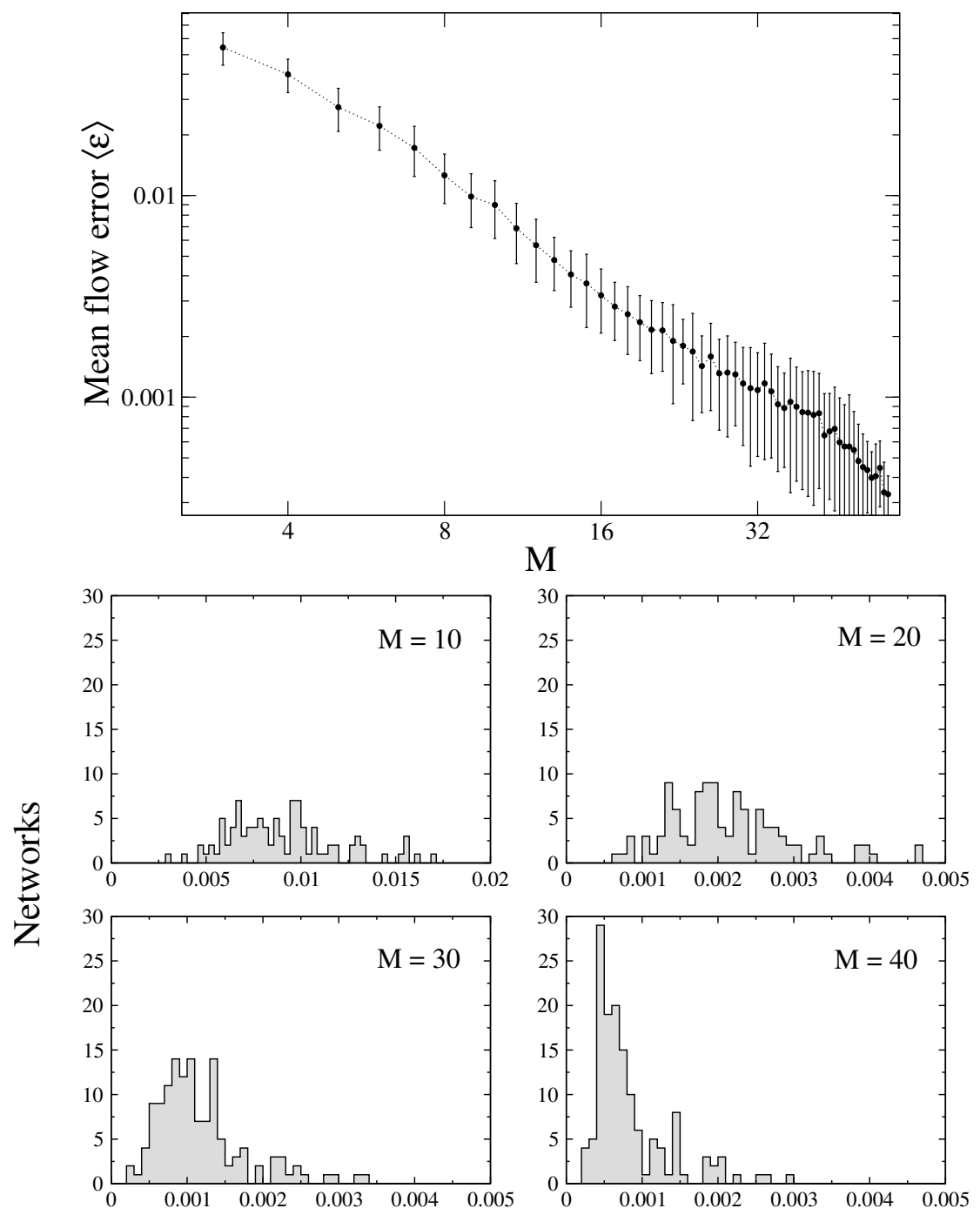

Flow error $\varepsilon$

Figure 3.5: (Top) Mean flow error $\langle\epsilon\rangle$ and its standard deviation for the ensembles of 100 networks as a function of the number $M$ of middle nodes. (Bottom) Distributions of flow errors in the ensembles of networks with different number of middle nodes.

Figure 3.5 shows mean flow errors and their standard deviations in the ensembles of designed networks as a function of the numbers of middle nodes $M$. We also present 
histograms of the flow errors, corresponding to the ensembles of networks designed with different numbers of middle nodes. It can be observed that the mean flow errors and the standard deviations decrease when the number of middle nodes increases. This behavior with respect to the number of middle nodes is general, independent of $N_{\text {in }}, N_{\text {out }}, K$, and the schemes of mutations used in the optimization process.

\subsubsection{Structural statistical properties of designed networks}

In this subsection we analyze structural statistical properties of the networks, which are constructed using evolutionary optimization algorithm with two different mutation schemes.

\section{Mean degree}

Figure 3.6 shows the average mean degree $\langle D\rangle$ as a function of the number of middle nodes $M$ for several ensembles of networks with different values of $K$. In Fig. 3.6a, networks are designed with path mutations. In Fig. 3.6b, they are obtained by using link mutations.
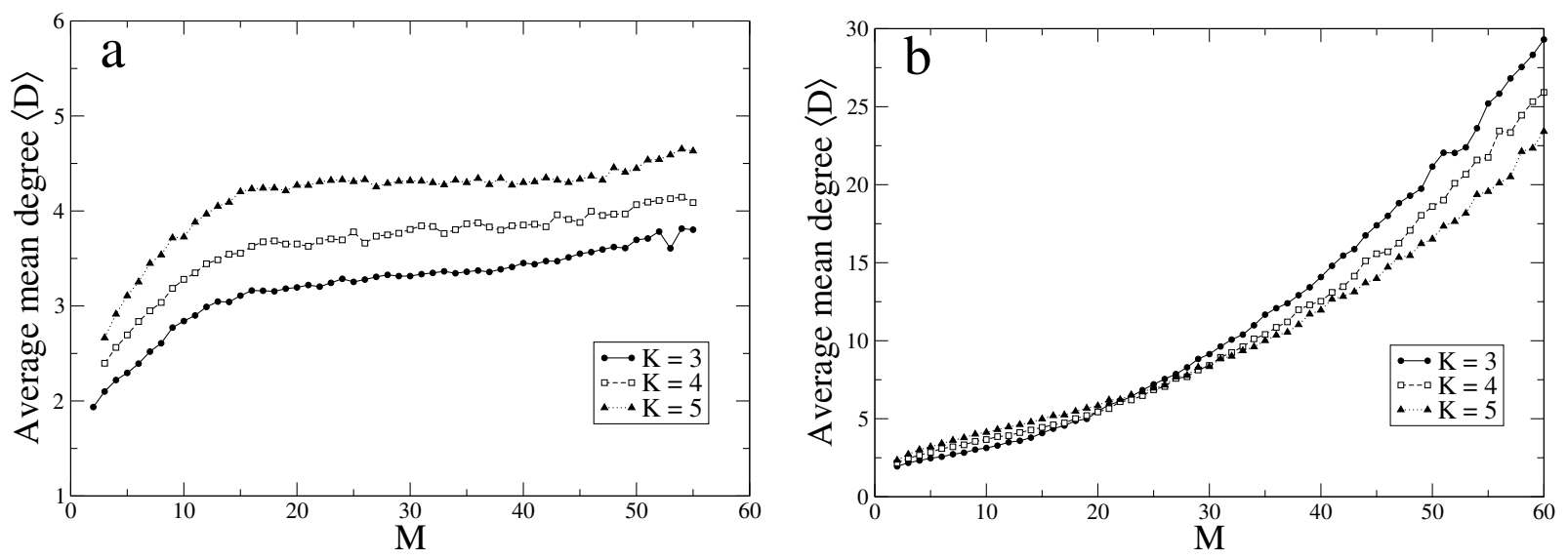

Figure 3.6: Dependence of the average mean degree $\langle D\rangle$ on the number $M$ of middle nodes for networks with different values of $K$, obtained by (a) path mutations and (b) link mutations.

We see that both mutation schemes generate networks with similar values of the mean degree when the number of middle nodes is smaller than 20. For the larger numbers $M$ of middle nodes, the mean degree of the networks obtained by link mutations is strongly increased as compared to that of the networks obtained with path mutations.

When the number $K$ of activated output nodes in the target output patterns is increased, the networks constructed using path mutations show larger values of $\langle D\rangle$. It can be understood, if we take into account that more connections are needed to activate 
more output nodes. However, in the networks constructed using link mutations, this relationship between $\langle D\rangle$ and $K$ holds only when the number of middle nodes is smaller than about 25. For larger values of $M$, the relationship is inverted and the networks with smaller $K$ have larger values of $\langle D\rangle$.

\section{Clustering coefficient}

Figure 3.7 shows the mean clustering coefficient $\langle C\rangle$ as a function of the number of middle nodes for the ensembles of networks obtained by path mutations (Fig. 3.7a) and by link mutations (Fig. 3.7b), using different values of $K$ in their target output patterns.

Networks, which are constructed using path mutations, have small clustering coefficients which decrease even more when we increase the number of middle nodes. When the number of middle nodes is larger than 10, $\langle C\rangle$ becomes independent of $K$ and the respective ensembles of networks behave in a similar way with respect to this property. For $M<10$, the mean value $\langle C\rangle$ grows with $K$.
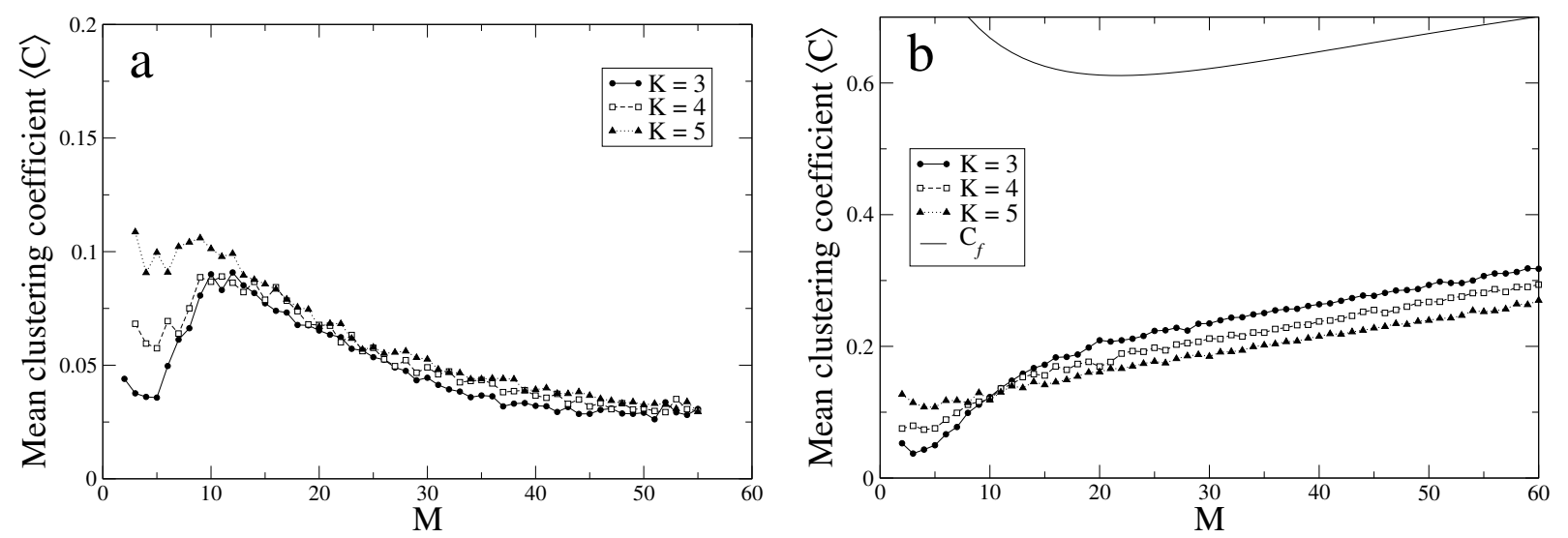

Figure 3.7: Mean clustering coefficients $\langle C\rangle$ of ensembles of networks obtained using (a) path mutations and (b) link mutations.

Networks obtained by link mutations show larger values of the clustering coefficient. When $M$ is smaller than about 10, we observe a behavior similar to that of the networks constructed using path mutations. However, for higher numbers $M$ of middle nodes the clustering coefficient grows linearly with $M$. We also see an inversion in the behavior with respect to $K$, since the networks with the smaller values of $K$ have larger values of $\langle C\rangle$.

Additionally, the clustering coefficient $C_{f}$ of the full-connected network for this model (following the connection rules) is shown by a solid line in Fig. 3.7b. It is given by

$$
C_{f}=\frac{1}{N}\left[N_{\text {in }}+N_{\text {out }}+\frac{M^{2}(M-1)}{N(N-1)}\right] .
$$

It can be seen that mean clustering coefficients $\langle C\rangle$ of the networks constructed using link mutations grow at the same rate for $M>10$ and their value is approximately $1 / 3$ of $C_{f}$. 


\section{Sizes of activated subnetworks}

An important property that characterizes our networks is the number of middle nodes $M_{i}$ which are activated by an input node $i$. Figure 3.8 shows the relative mean number $\left\langle M_{i}\right\rangle / M$ of the activated middle nodes as a function of the total number $M$ of middle nodes for different network ensembles. Note that $\left\langle M_{i}\right\rangle$ represents the mean size of an activated subnetwork.
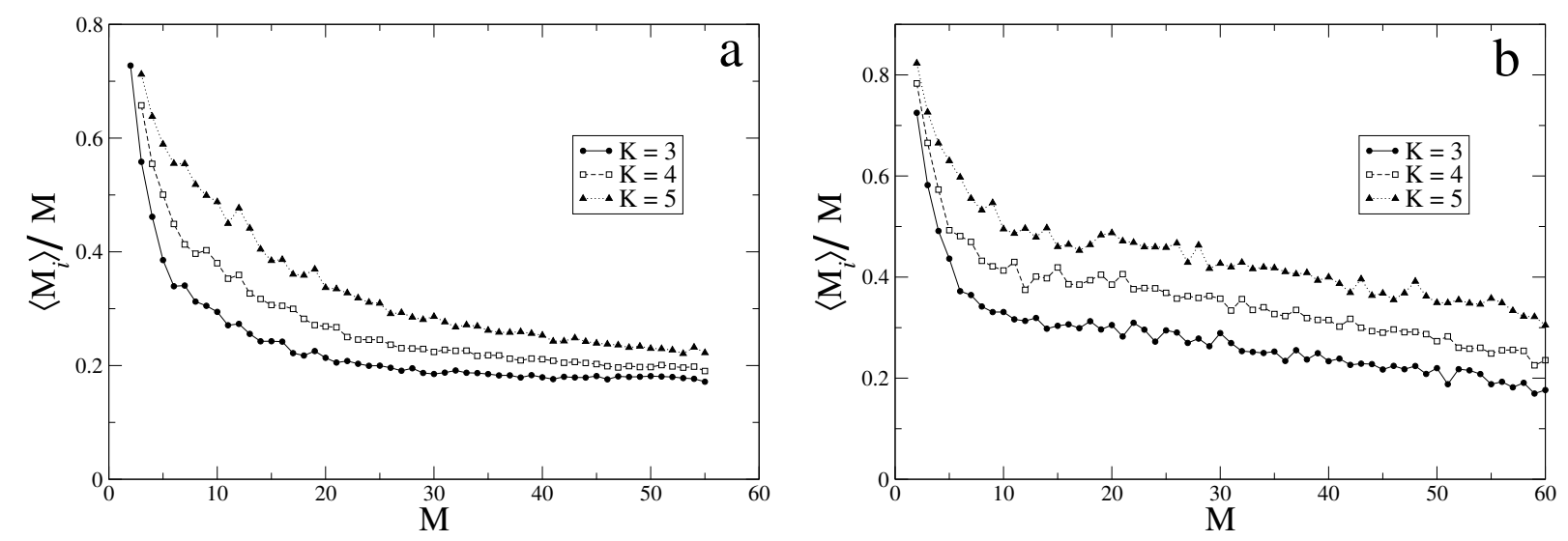

Figure 3.8: Dependences of the relative mean number of middle nodes, activated per input node, on the total number of middle nodes. (a) Path mutations, (b) link mutations.

Similar behavior is observed in the networks constructed using different mutation schemes and with different values of $K$ in their target output patterns. When networks have only a few middle nodes, each input node activates practically the whole network. The fraction of activated nodes decreases when the number of middle nodes of the networks is increased.

Thus, this property reveals overlaps between subnetworks activated by different input nodes. For both mutation schemes, the overlaps increase with $K$, since networks need then to activate more output nodes.

The sizes of activated subnetworks are larger in the networks constructed using link mutations, as compared to the path mutation scheme. This can be related to the fact that, for the the link mutation scheme, networks have more connections and their architecture is highly clusterized. In this case, both the clustering coefficient and the mean degree increase when the number of middle nodes grows, so that clusters are characteristic for the activated subnetworks.

\section{Degree distribution}

Now we analyze degree distributions of the networks designed using different mutation schemes. All considered networks have the same numbers of nodes and the same number 
of activated nodes $K=4$ in their target output patterns. Figure 3.9 shows degree distributions of the respective ensembles.

Path mutations
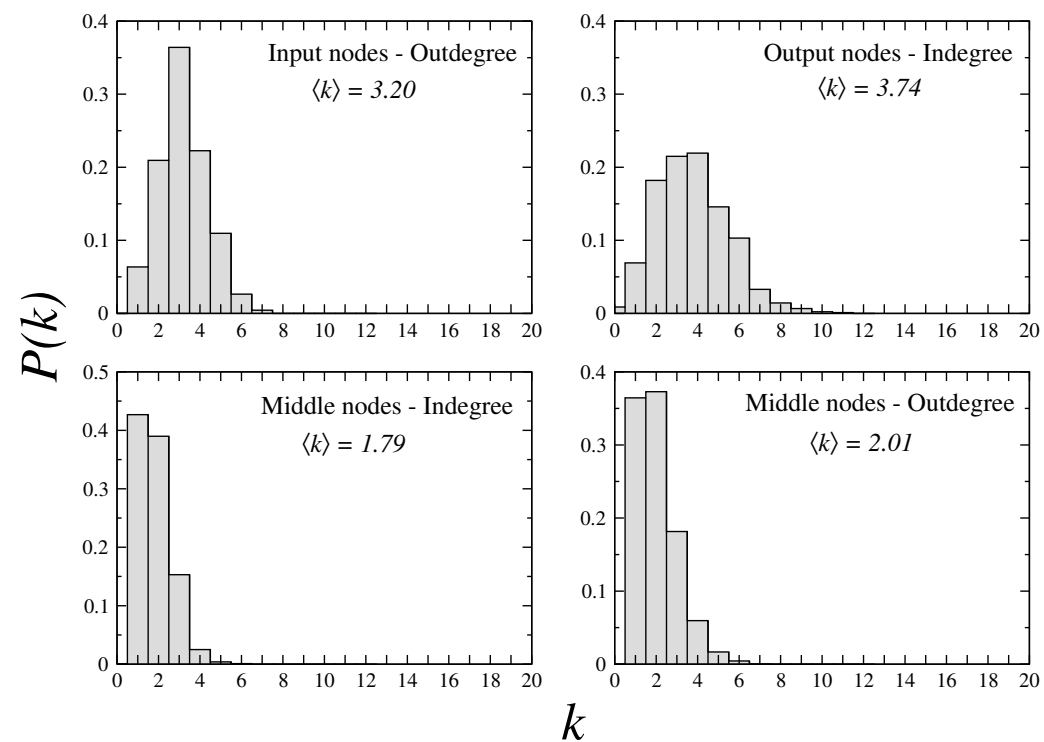

Link mutations

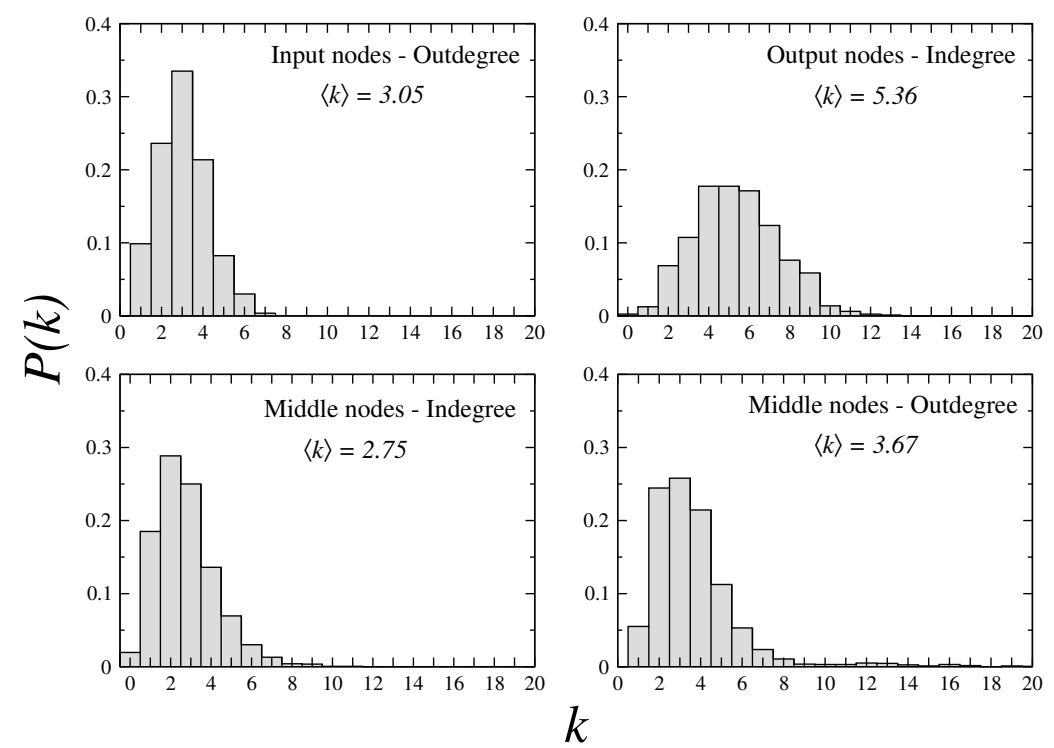

Figure 3.9: Degree distributions of two ensembles of 100 networks each, which have been designed by using path mutations (top) and link mutations (bottom).

The outdegree distributions of the input nodes are practically the same in both ensembles of networks. In both cases, the mean values of the distributions are close to the value $M / N_{\text {in }}$ (2.5 in these networks).

In the case of middle nodes, there are two associated distributions of indegrees and outdegrees. In the ensemble of networks obtained with path mutations, both distributions 
have similar shapes and their mean values are close to 2 . This indicates the existence of only few connections between the middle nodes, in agreement with small values of the clustering coefficient and the small mean size of the activated subnetworks.

In the ensembles of networks constructed using link mutations, the indegree and the outdegree distributions also have similar shapes, with the mean values of 2.74 and 3.67, respectively. These relatively high numbers indicate the existence of many connections between the middle nodes, in comparison with the previous case. Moreover, sometimes we find nodes with 12, 16 and more outgoing connections. Taking into account the total size of the network $(N=36)$, such values are very special. By constructing the joint distribution $P\left(k_{\text {in }}, k_{\text {out }}\right)$ of indegree and outdegree (not shown), we have found that such values correspond to the nodes without input connections. Because there is no flux feeding them, they have no influence on the output. The existence of such nodes can be understood if we note that, by using link mutations, it is possible to add an outgoing connection to such nodes and the resulting $\Delta \epsilon$ would be zero, so that such a mutation is always accepted. However, adding an input connection that comes from an active node produces a large value of $\Delta \epsilon$, since this node is connected practically to the whole network. Therefore, such mutation would almost always be rejected.

The indegree distributions of the output nodes are similar in both ensembles. However, the mean indegree value of the networks constructed using link mutations $(\langle k\rangle=5.36)$ is larger than in the networks constructed using path mutations $(\langle k\rangle=3.74)$. The higher number of incoming connections is related to the larger sizes of the subnetworks activated by an input node. Obviously, output nodes are fed from many connections when subnetworks are large.

\subsubsection{Motif distributions}

In this subsection, the mean normalized Z scores (motif distributions) for subgraphs of three nodes in the ensembles of designed networks are determined. First, we explain how these distributions are constructed. Then, we show motif distributions of ensembles of designed networks with different sizes and optimized by using different mutation schemes.

\section{Motif distributions of a network and of an ensemble}

To determine motif distributions of networks, we have used the software MFinder provided by Uri Alon ${ }^{2}$. For each network, this software determines its motif distributions with respect to motifs of different sizes. In the case of three-node motifs, the vector of normalized score $\vec{z}$ has 13 elements. To calculate this vector, the program uses an ensemble of 1000 networks, where each network is a different randomized version of the given network. The definition of the normalized score vector was given in Chapter 2 (see

\footnotetext{
${ }^{2}$ This software can be found in the web page of Uri Alon, in the Weizmann Institute (www.weizmann.ac.il/mcb/UriAlon/).
} 
equation 1.14).

For an ensemble of $\mathcal{N}$ networks, we compute its average normalized Z score $\langle\vec{z}\rangle$ as the average of the vectors $\vec{z}$ of the networks in the ensemble, according to

$$
\langle\vec{z}\rangle=\frac{1}{\mathcal{N}} \sum_{i=1}^{\mathcal{N}} \overrightarrow{z_{i}}
$$

and its standard deviation vector

$$
\vec{\sigma}=\sqrt{\frac{1}{\mathcal{N}} \sum_{k=1}^{\mathcal{N}}\left(\langle\vec{z}\rangle-\overrightarrow{z_{i}}\right)^{2}}
$$

The vector $\langle\vec{z}\rangle$ cannot be compared easily with the vectors charactering motif distributions of other networks, because it is not normalized to unity. We perform such normalization and determine the mean normalized score vector $\langle\vec{Z}\rangle$, whose elements are

$$
\langle Z\rangle_{\alpha}=f_{\alpha}(\langle\vec{z}\rangle)=\frac{\langle z\rangle_{\alpha}}{\sqrt{\sum_{\beta=1}^{13}\langle z\rangle_{\beta}^{2}}} \quad(\alpha=1, \ldots, 13) .
$$

An element $\langle Z\rangle_{\alpha}$ of the mean normalized score vector has a statistical dispersion $\Sigma_{\alpha}$ which is a function of the vectors $\langle\vec{z}\rangle$ and $\langle\vec{\sigma}\rangle$ and it is calculated making error propagation according to:

$$
\Sigma_{\alpha}=\sum_{\beta=1}^{13}\left|\frac{\partial f_{\alpha}(\vec{x})}{\partial x_{\beta}}\right|_{\vec{x}=\langle\vec{z}\rangle} \sigma_{\beta} \quad(\alpha=1, \ldots, 13) .
$$

The mean normalized score vector $\langle\vec{Z}\rangle$ and its dispersion $\vec{\Sigma}$ characterize the motif distribution of an ensemble. To simplify the notation, when we refer to an ensemble, we just call these vectors the normalized $Z$ score and the statistical dispersion of the motif distribution of the considered ensemble.

\section{Motif distributions of the designed networks}

Figure 3.10 shows motif distributions of several ensembles which have different numbers of middle nodes and are designed by using different mutation schemes. Networks with $M=10$ show similar motif distributions for both mutation schemes. Earlier, we have seen that the structural properties are also very close for both mutation schemes, when the networks have only a few middle nodes.

When $M=30$, motif distributions of the ensembles, obtained using different mutation schemes, are still similar. However, the standard deviations are much larger now in the networks constructed by path mutation scheme. The large dispersion is, in part, due to the fact that the networks have less connections and, therefore, less motif subgraphs for this mutation scheme. Moreover, we will later see that these ensembles actually consist of several sub-ensembles with very different well defined motif distributions, depending on their robustness properties. 
Path mutations

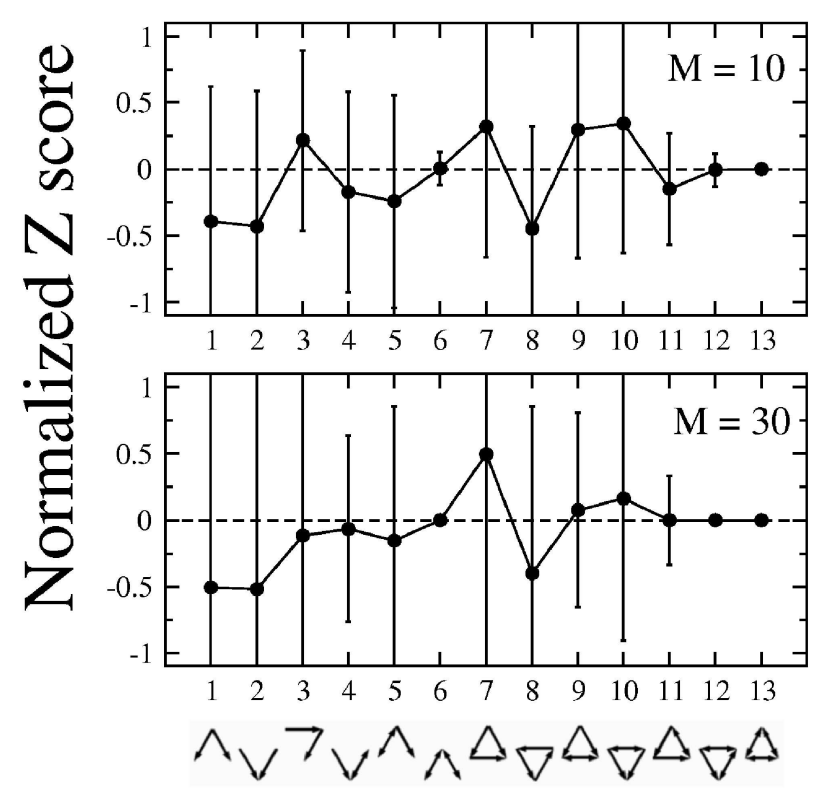

Subgraphs
Link mutations
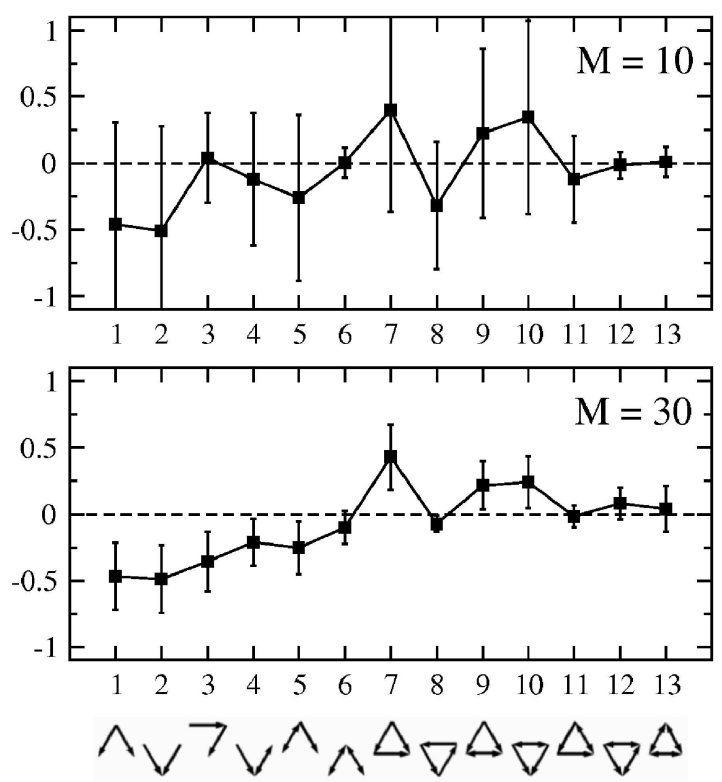

Subgraphs

Figure 3.10: Mean motif distributions of the designed networks with different numbers $M$ of middle nodes for two different schemes of evolutionary mutations. Each ensemble contains 100 networks, obtained by running evolution with a different, randomly chosen initial condition and a different, randomly chosen target pattern. The parameters $K=4, N_{\text {in }}=N_{\text {out }}=8$ are the same for all networks. Bars show statistical dispersion of data. 


\section{Chapter 4}

\section{Functional networks robust against local damage}

In this chapter, we study how pipeline networks can be designed to be functional and, moreover, robust against local damage, such as the removal of nodes or connections. In the first part, we introduce the definition of robustness based on the requirement that a network must maintain its functionality when a damage affects its structure. A modified version of the algorithm used earlier to construct functional networks is presented, with the aim not only to reduce the flow error, but also to increase robustness of evolving networks. In the second part, principal properties of the networks optimized using different criteria of robustness (different kinds of local damages) are compared.

\subsection{Definition of robustness}

Real biological networks are able to operate despite local damages, such as deletions of single nodes or links. This means that they can retain their required function despite random local damage. To study functional robustness and to design functional networks robust against local damage, we need a definition of functional robustness.

We take a network to be functional, if its flow error with respect to its target output pattern $\mathbb{Q}^{0}$ is smaller than a certain threshold $h$. With this criterion, we can check whether a network retains its functionality when some damage has been applied to it.

Two possible kinds of local damages in the networks are considered: the deletion of a randomly chosen middle node and the deletion of a randomly chosen connection. Applying a damage to a network $G$ with the flow error $\epsilon$, a new network $G^{\prime}$ with the flow error $\epsilon^{\prime}$ is obtained. We say that the damage has been abortive if the flow error $\epsilon^{\prime}$ is larger than the tolerance threshold $h$, so that the network becomes non-functional.

The damage shell $S$ of the network $G$ is a set of all networks obtained by applying to it all possible local damages (removal of single connections or nodes). We define functional 
robustness $\rho$ of network $G$ as the fraction of all networks in the damage shell which are not abortive,

$$
\rho(G)=\frac{1}{N_{S}} \sum_{G^{\prime} \in S} \Theta\left(h-\epsilon^{\prime}\right)
$$

where $N_{S}$ is the total number of networks in the damage shell $S$ and $\Theta(z)$ is the step function, such that $\Theta(z)=1$ for $z>0$ and $\Theta(z)=0$ otherwise.

\subsection{Evolutionary optimization}

The evolutionary optimization method used to design functional robust networks will be similar to that previously used to construct functional networks. Because we want not only to minimize the flow error, but also to increase the robustness, some modifications are however needed. Optimizations will be performed according to a criterion of robustness given by the kind of local damage taken. If we consider the removal of a node, we say that the optimization is by node robustness, whereas if the criterion refers to the deletion of a connection, the optimization is by link robustness.

\subsubsection{Optimization algorithm}

Suppose that in an iteration we have some network $G$ with flow error $\epsilon$ and robustness $\rho$. Applying to $G$ an evolutionary mutation, we get new network $G^{\prime}$ with flow error $\epsilon^{\prime}$ and robustness $\rho^{\prime}$. The selection depends on the value of $\epsilon$ with respect to the threshold $h$. If $\epsilon>h$, the network is still non-functional and we only minimize the flow error. If $\Delta \epsilon=\epsilon^{\prime}-\epsilon<0$, we accept the mutation, otherwise the mutation is accepted with a probability $\exp \left(-\Delta \epsilon / \sigma_{E} \epsilon\right)$, where $\sigma_{E}$ is the temperature parameter. If $\epsilon<h$, the network is already functional and the optimization is switched to robustness. Now, the mutation is accepted, if $\Delta \rho=\rho-\rho^{\prime}<0$, and accepted with probability $\exp \left(-\Delta \rho / \sigma_{R}(1-\rho)\right)$, if $\Delta \rho>0$. Here $\sigma_{R}$ is the second temperature paramater. In the next iteration, an evolutionary mutation is applied to the previouly accepted network and the same selection procedure is taken.

With this algorithm, we switch the selection criterion between the minimization of the flow error and the maximization of the the robustness, according to the flow error of a network with respect to the threshold $h$. In the beginning of the evolutionary process, we mostly minimize the flow error in order to obtain functional networks. In the final stage, robustness is maximized. Note that changes between different criteria can occur several times during an evolution.

As the initial network in the optimization process, random Erdös-Rényi networks with different connectivities $p$ are taken. The connectivity $p$ of an initial network is chosen at random from the interval between 0 and 1 in each evolution.

When ensembles of designed networks are constructed to investigate their statistical 
properties, we choose at random the target output pattern in each individual evolutionary optimization process. The target output patterns are generated using the same algorithm as in Chapter 3.

\section{Evolutionary mutations}

To determine robustness, flow errors of all networks in the damage shell of a given network must be computed in each iteration step. The number of the networks in the damage shell depends on the kind of damage considered. When middle nodes are deleted, there are $M$ networks in the shell. When links are deleted, the size of the damage shell depends on the network architecture and can be even much larger. Typically, our evolving networks have $M=20$ middle nodes and about 150 connections. A single evolution usually takes anout $10^{5}$ iterations steps.

Maximization of robustness is the most computationally intensive part of the optimization process, if we compare it with the minimization of the flow error. Only evolutionary path mutations will be used to design networks in this chapter. We have previously seen that they yield faster optimization convergence than link mutations. Moreover, the link mutation scheme tends to generate architectures with a large number of connections, which makes the estimation of robustness againt link removal even more time consuming.

\section{Example of network construction}

Figure 4.1 shows an example of evolution where the network has been optimized for robustness against node deletions. We see that the flow error is minimized until the threshold $h$ is reached at $t_{1}=6486$. After that, the evolution is switched to maximize the robustness of the network. At this stage, the flow error can change freely provided that it stays under the threshold $h$. Below in this figure, we show the final network, obtained after $2 \times 10^{5}$ iterations. Its robustness has been increased to 0.9, as compared with the robustness of about 0.1 when the robustness optimization has been started.

\subsubsection{Ensembles of networks}

We have constructed two ensembles with 100 networks in each of them. In the first ensemble, the networks evolved only to minimize their flow errors. The second ensemble is optimized for robustness against the removal of nodes. In both cases the evolutions are ended after $3 \times 10^{5}$ iterations. The same parameter $N_{\text {in }}=8, M=20, N_{\text {out }}=8$ and $K=4$ are used in all evolutions.

The effects of the optimization by robustness are clearly seen in the distributions of the flow errors of the obtained networks and of the networks in their damage shells (Fig. 4.2). Empty black distributions correspond to flow erros of the networks of the ensembles. They are similar for both cases. However, the distribution of flow errors after the damages are 
a

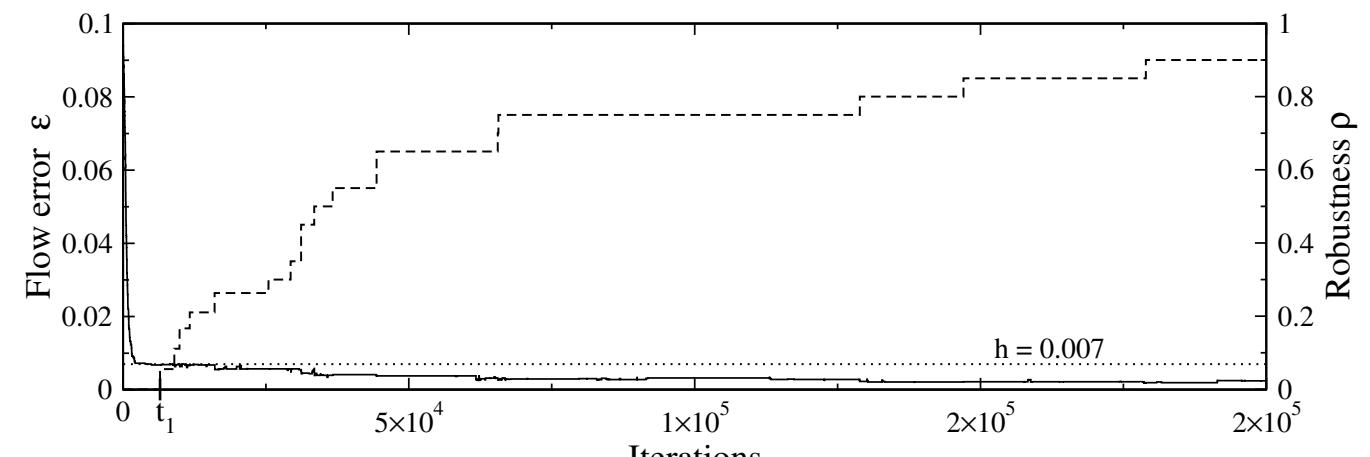

b

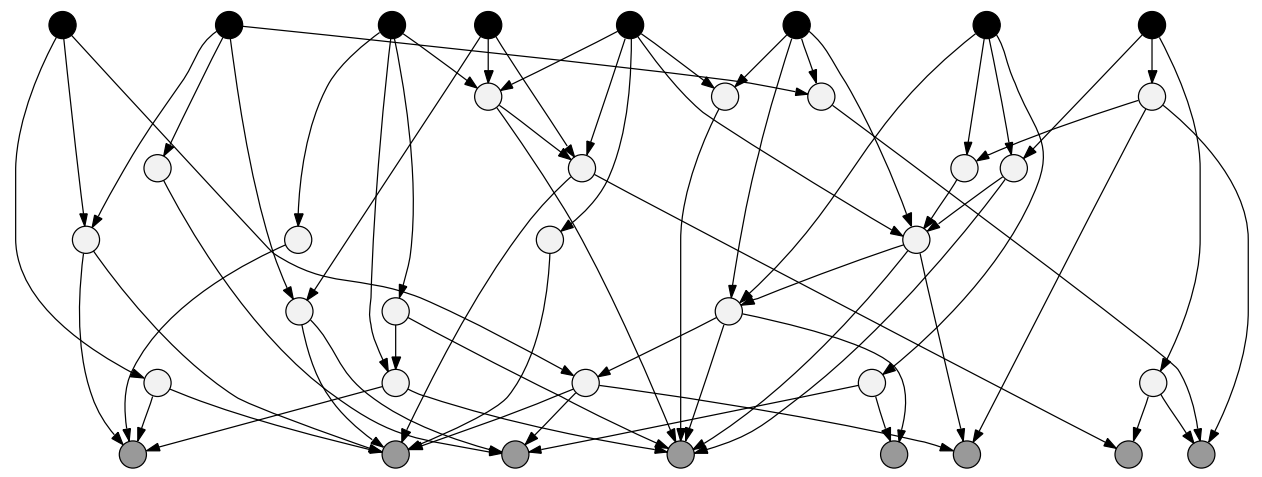

Figure 4.1: (a) Evolution of the flow error (solid line) and the robustness against removal of nodes (dash line) in the optimization process. (b) Final obtained network (b). The parameters used are $h=0.007, N_{\text {in }}=8, M=20, N_{\text {out }}=8, K=4$ and $\sigma_{E}=\sigma_{R}=1 \times 10^{-4}$.
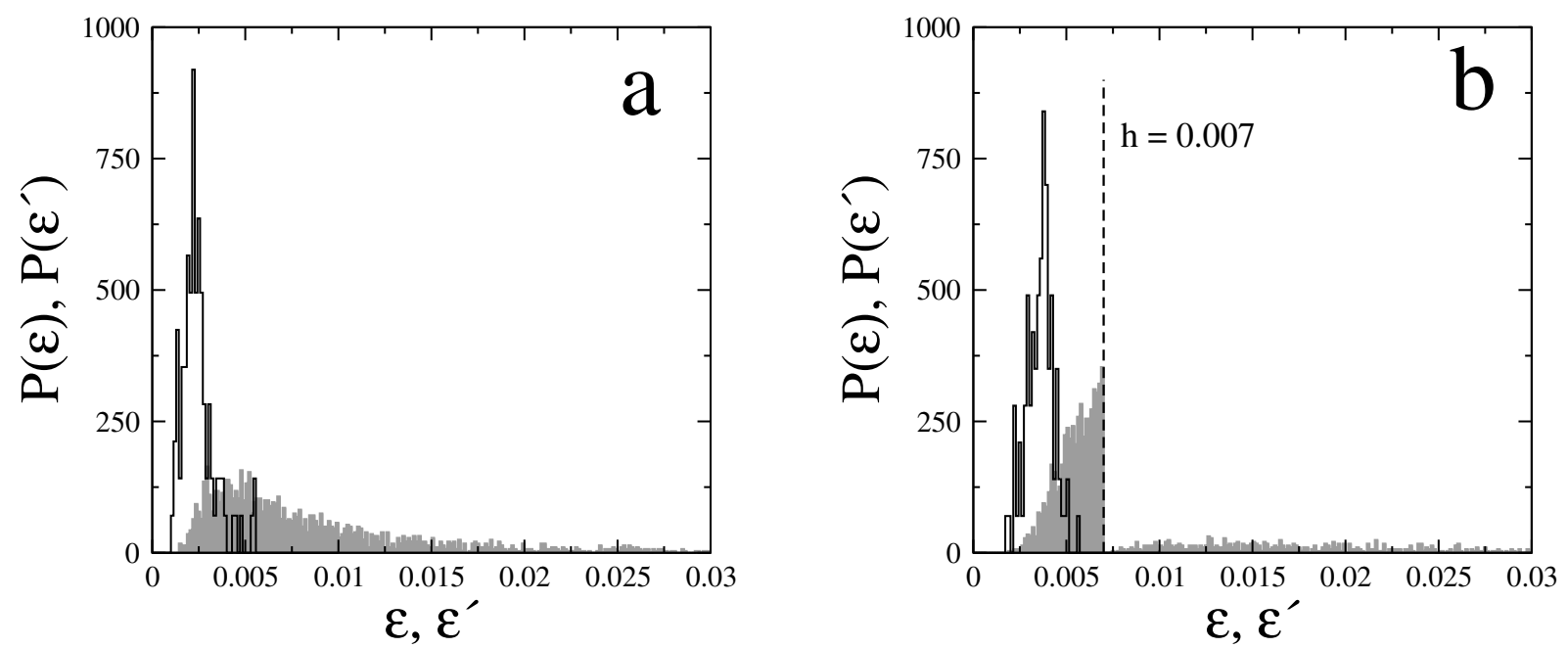

Figure 4.2: Distributions of flow errors $\epsilon$ in the ensembles of 100 networks (black), optimized only by flow error (a) and optimized for robustness against deletion of a node (b). Gray histograms show distributions of flow errors $\epsilon^{\prime}$ of the networks in the damage shells (after removing a node). 
completely different in these two ensembles. The networks optimized only by flow error yield a broad distribution with a long tail extending to large flow errors. In constrast to this, flow errors exceding the threshold $h$ become strongly suppressed in the damage shells of the networks optimized to increase their robustness.

The black distributions are constructed normalizing the histograms of flow errors of the networks of the ensembles. The gray distributions are obtained normalizing the histograms constructed computing the flows errors of the networks of the damage shells of the optimized networks. Since we have $M=20$ middle nodes in the example, the damage shell of each optimized network has 20 networks. Therefore, the histograms, used to construct the gray distributions, are based on $100 \times M=2000$ networks.

Mean robustness of an ensemble of networks can be defined as

$$
\langle\rho\rangle=\int_{0}^{h} P\left(\epsilon^{\prime}\right) d \epsilon
$$

where $P(\epsilon)$ is the distribution of flow errors in the damage shells of networks in the considered ensemble and $h$ is the threshold used to evaluate the robustness. The mean robustness of the networks in the ensemble optimized for deletion of nodes is $\langle\rho\rangle=0.87$, as compared to the much smaller value of $\langle\rho\rangle=0.53$ for the networks optimized only with respect to their flow errors.

\subsubsection{Temperature parameters $\sigma_{E}$ and $\sigma_{R}$}

The optimization algorithm has two temperatures parameters $\sigma_{E}$ and $\sigma_{R}$. The effects of the first of them $\left(\sigma_{E}\right)$ have been studied previously in Chapter 3 and its optimal value has now been used in the optimizations. We have performed a similar study for the temperature parameter $\sigma_{R}$ (Fig. 4.3).

The optimization works well only when $\log \left(\sigma_{R}\right)<10^{-2}$ for both kinds of optimization. It is interesting to note that these critical values are the same as those found for the optimization by only flow error (Fig. 3.4a) in the previous Chapter 3. This result is natural if we take into account that an increase in the robustness of a network can be seen as a reduction of the flow errors of all networks in its damage shell. Since $\sigma_{E}$ and $\sigma_{R}$ have similar critical values, we take $\sigma=\sigma_{R}=\sigma_{E}$ in all subsequent simulations.

\subsection{Statistical properties of designed robust networks}

To study how the requirement of robustness affects network properties, many simulations for ensembles of 100 networks have been performed, choosing different thresholds and different kinds of local damages. All networks had $N_{\text {in }}=8, M=20$ and $N_{\text {out }}=8$, and their target output patterns had $K=4$. The temperature parameter was $\sigma=10^{-3}$. The evolutions were always terminated after $3 \times 10^{5}$ iterations. 


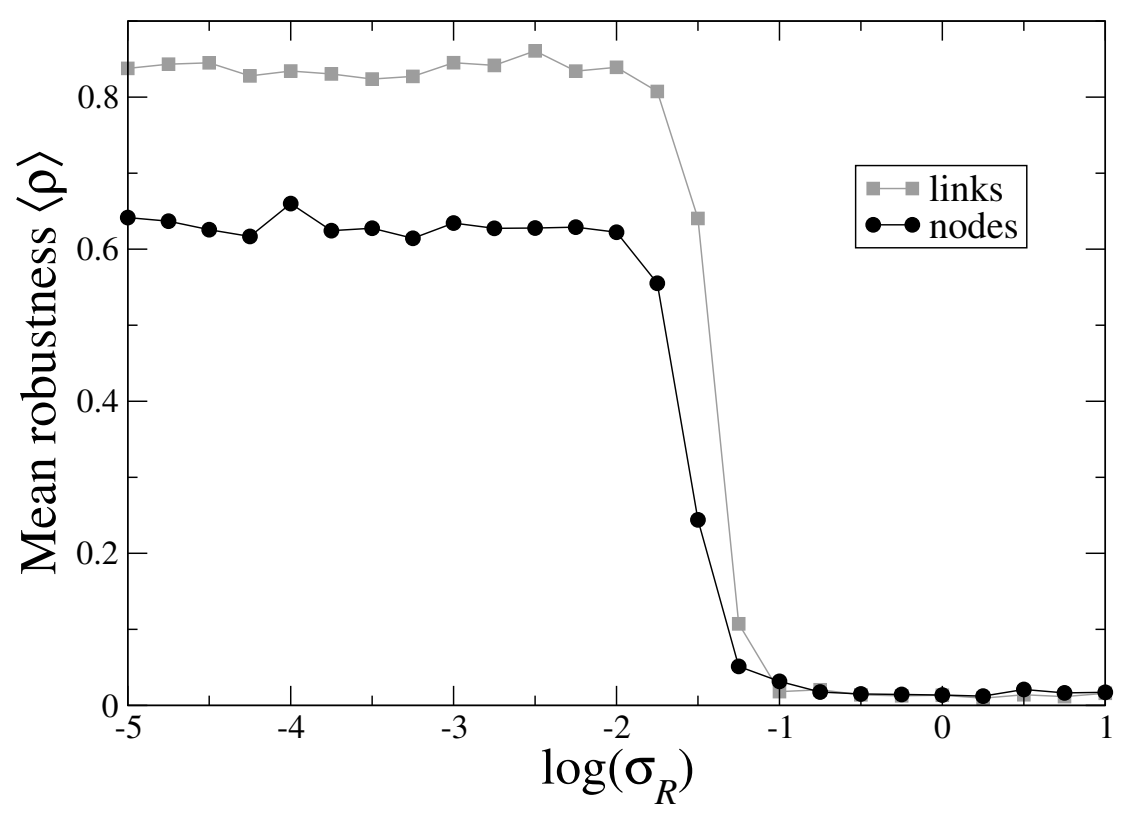

Figure 4.3: Mean robustness of the ensembles of 100 networks optimized against deletion of nodes (circles) or links (squares) as a function of the temperature parameter $\sigma_{R}$. All networks have $N=36$ with $N_{\text {in }}=8, N_{\text {out }}=8, M=20$, and the output target patterns have $K=4$. The simulation parameters are $\sigma_{E}=10^{-3}, h=0.007$. The evolutions were terminated after $10^{5}$ iterations.

Statistical properties studied are defined as averages over the ensemble of networks. In such averages, only functional networks with $\epsilon<h$ are included. Therefore, the actual number of networks in an ensemble may be less than 100, since some of the networks have larger values of flow errors and are discarded.

\subsubsection{Distribution of flow errors}

In the optimization by robustness, unlike the optimization by only the flow error, we do not need to minimize the error of the networks as much as possible, since the networks are aready functional when their errors are smaller than the threshold. Therefore, mean flow errors of the ensembles of networks are just below the threshold in this case. Figure 4.4 displays the mean flow error of ensembles of networks optimized for link and node robustnenss.

Optimization for robustness has a strong effect on the distribution of flow errors of the networks in the damage shells. Figure 4.5 presents such distributions for ensembles of networks optimized for link robustness, using different threshold values.

The distributions show jumps at the thresholds. The part of the distribution, which corresponds to errors exceeding the threshold, is reduced. When the threshold is small ( $h=0.003)$, the reduction is weak and the distribution is still close to that of the net- 


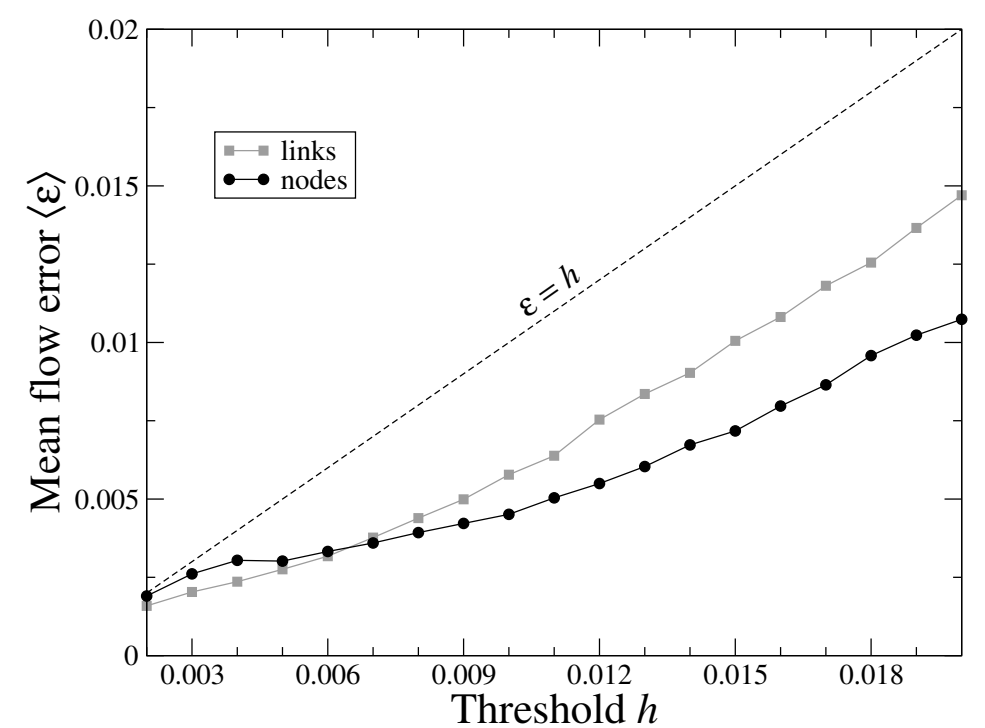

Figure 4.4: Mean flow errors of the ensembles of networks optimized for node robustness (circles) and for link robustness (squares) as function of the threshold used.
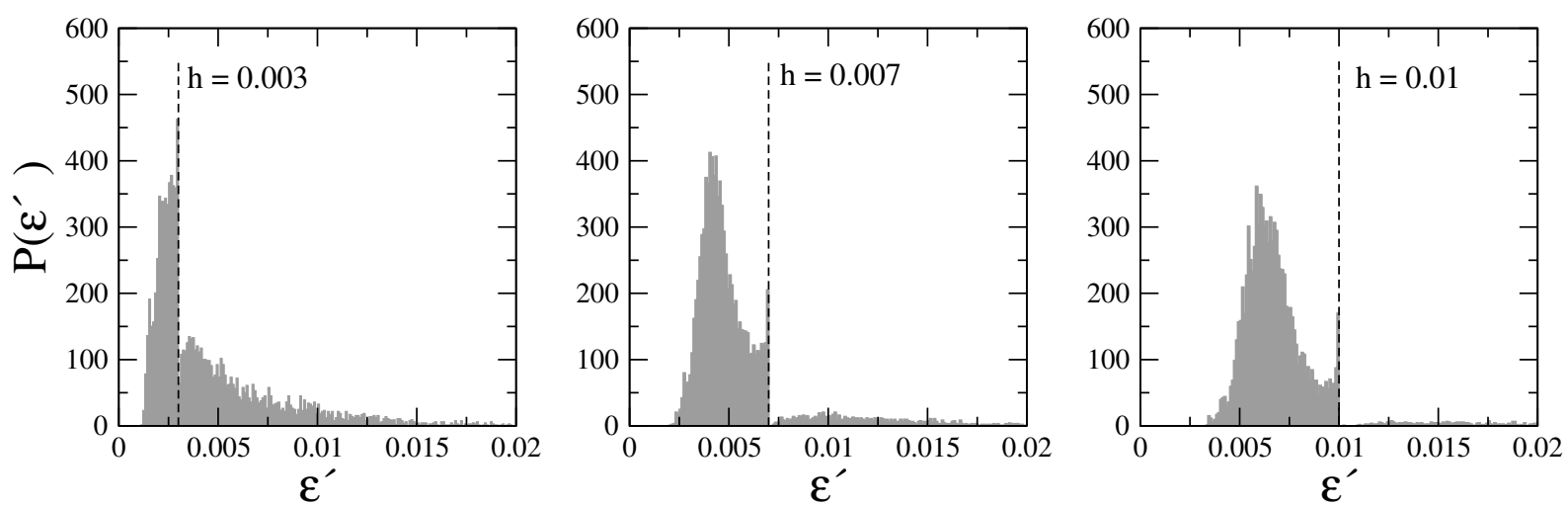

Figure 4.5: Distributions of flow errors in the damage shells (removing a connection) of networks optimized with respect to link robustness, using different thresholds $h$.

works optimized only by flow error (see Fig. 4.2a). A large number of networks in the damage shell has errors exceeding the threshold in this case. On the other hand, when the threshold is large $(h=0.01)$, the optimization only affects the tail of the distribution. The most interesting behaviour is found when the threshold has a value close to the mean value of the distribution of the damage shell of the networks optimized only by flow error $(h=0.007)$. Then, networks with the errors larger than the threshold almost disappear and an increase of the robustness after the optimization is significant. 


\subsubsection{Mean robustness}

Robustness against different kinds of damages can be determined for any network, independent of the criterion used during the optimization process. Thus, for example, we can consider node robustness of the networks optimized only by flow error, or of the networks optimized for link removals. Comparing robustness in ensembles of networks optimized by different criteria allows us to quantify the performance of the robustness optimization process.

Below, special notations will be used. The subscript indicates the criterion used during the optimization process ( $n$ for node robustness, $l$ for link robustness and $f$ for only flow error), the superscript indicates the criterion of robustness used in the evaluation. Thus, $\rho_{f}^{n}$ is the robustness against node removals of a network optimized only for flow error and $\rho_{n}^{l}$ is the robustness against link removals of a network optimized for node removals. When both indices are the same, we use only the subscript. For example, $\rho_{l}$ is the robustness against link deletions of a network optimized for link robustness.

Another point to consider is that the threshold used during the optimization process $\left(h_{\text {opt }}\right)$ can be different from the threshold used in the evaluation of the robustness $\left(h_{\text {rob }}\right)$. In order to avoid confusion, we always evaluate the robustness with the same value of threshold that has been used during the optimization process $\left(h=h_{\text {opt }}=h_{\text {rob }}\right)$.

Figure 4.6 shows the dependence of the mean node robustness of several ensembles of networks on the threshold. The networks optimized against node removals (circles) have

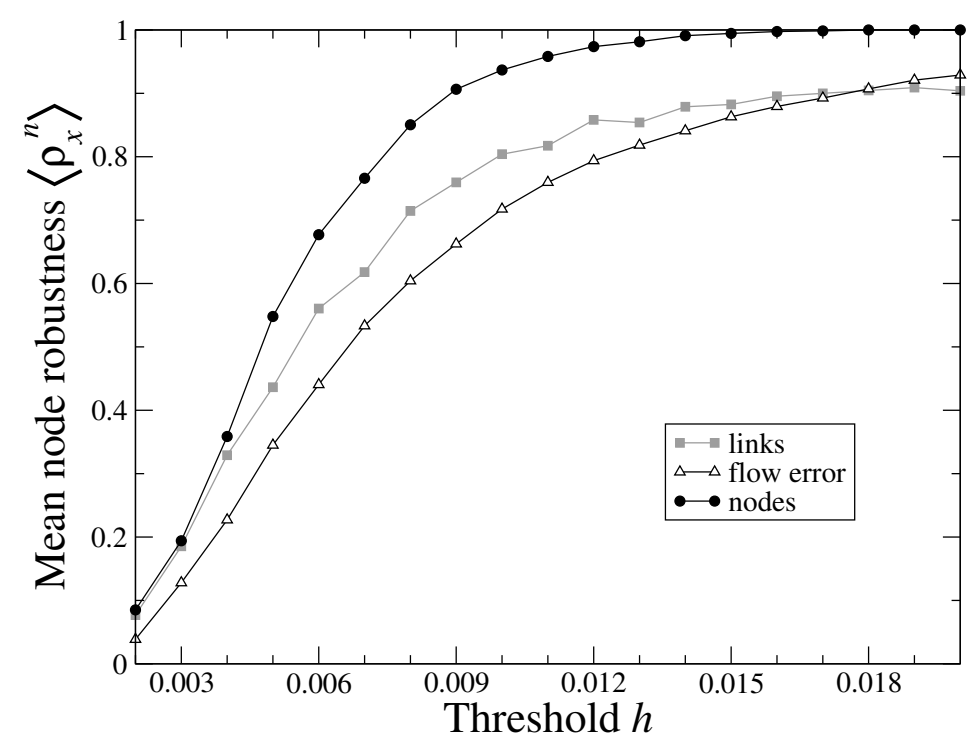

Figure 4.6: Mean node robustness $\left\langle\rho_{x}^{n}\right\rangle$ as a function of the threshold for ensembles of networks optimized for robustness against node or link deletions, or only by the flow error $(x=n, l, f))$. Averages over ensembles of 100 networks at each point.

node robustness larger than the networks optimized only by flow error (triangles) and the networks optimized for link robustness (squares). The networks, optimized for link 
deletions, already have higher node robustness than the networks optimized only by flow error. However, it is still smaller than that of the networks optimized specifically for node deletion. The strongest increase of the node robustness after the optimization is obtained in the interval between $h=0.005$ and $h=0.014$.

Figure 4.7 displays mean link robustness of ensembles of networks optimized using different criteria. Similar to the previous case, the networks optimized for link robustness

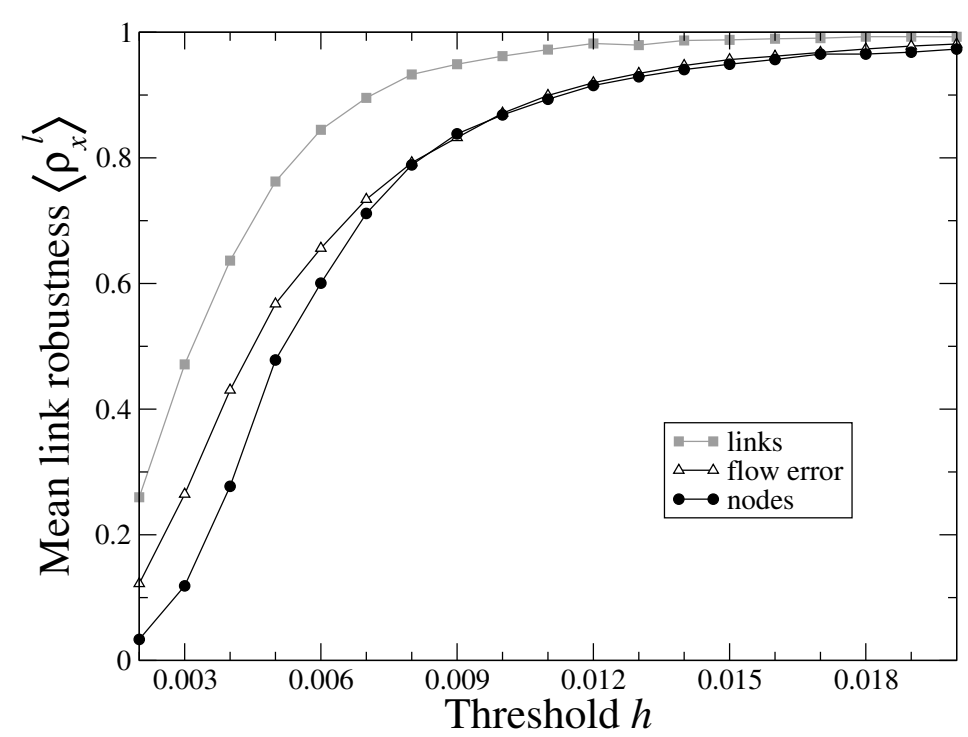

Figure 4.7: Mean link robustness $\left\langle\rho_{x}^{l}\right\rangle$ as a function of the threshold for ensembles of networks optimized using different criteria $(x=n, l, f))$. Averages over ensembles of 100 networks at each point.

(squares) show larger values of this kind of robustness than the networks optimized for node robustness (circles) and the networks optimized only by flow error (triangles). Here, the networks optimized only by flow error and for node robustness, have close values of link robustness when $h>0.007$. For lower thresholds, the networks optimized for node robustness are even less robust against the deletion of a connection than the networks optimized only by flow error. The optimization using this criterion is most efficient in the interval of thresholds between $h=0.002$ and $h=0.014$.

\subsubsection{Structural statistical properties of robust networks}

In the previous section, we have seen that optimization by robustness generates important changes in the distributions of flow erros of the networks of the damage shell, as compared with the networks optimized only by flow error. Now, we shall investigate how this optimization affects architecture of the networks. 


\section{Mean degree}

Figure 4.8 shows average mean degrees of ensembles of networks optimized by different criteria. The mean degrees (i. e., mean numbers of connections per node) are strongly

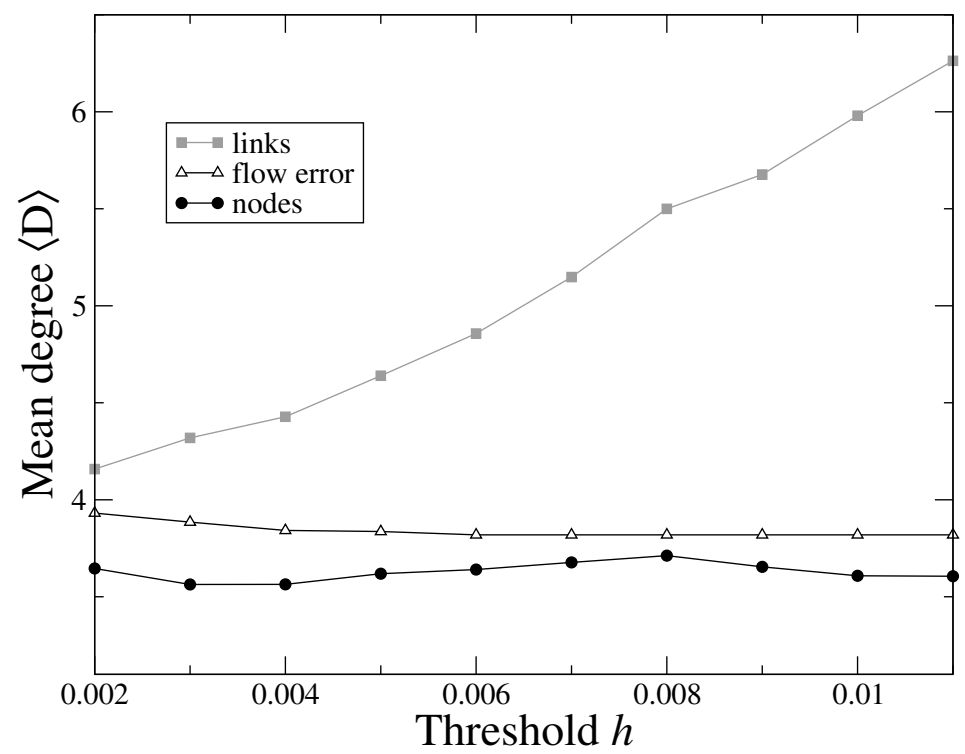

Figure 4.8: Mean degrees of ensembles of networks optimized by node and link removals and only by flow error as a function of the threshold.

increased in the networks optimized for link robustness (squares) as compared to the networks optimized only by the flow error (triangles). The difference grows with the threshold.

In the case of the networks optimized for node robustness, the mean degrees (circles) are smaller than those of the networks optimized only by flow error. However, the decrease is not strong and no sensitive dependence on the threshold is observed here.

\section{Clustering coefficient}

In Fig. 4.9, mean values of the clustering coefficient of ensembles of networks, optimized by different criterions of robustness and for different thresholds, are shown. Networks optimized for link robustness (squares) have larger clustering coefficients than the networks optimized using the other two criteria. The difference increases with the threshold.

Clustering coefficients of the networks, optimized for node robustness, are smaller than those of the networks optimized only by flow error. Moreover, they decrease with the threshold. This behaviour is interesting, since the number of connections (the mean degree) does not show a clear dependence on the threshold used (see Fig. 4.8). 


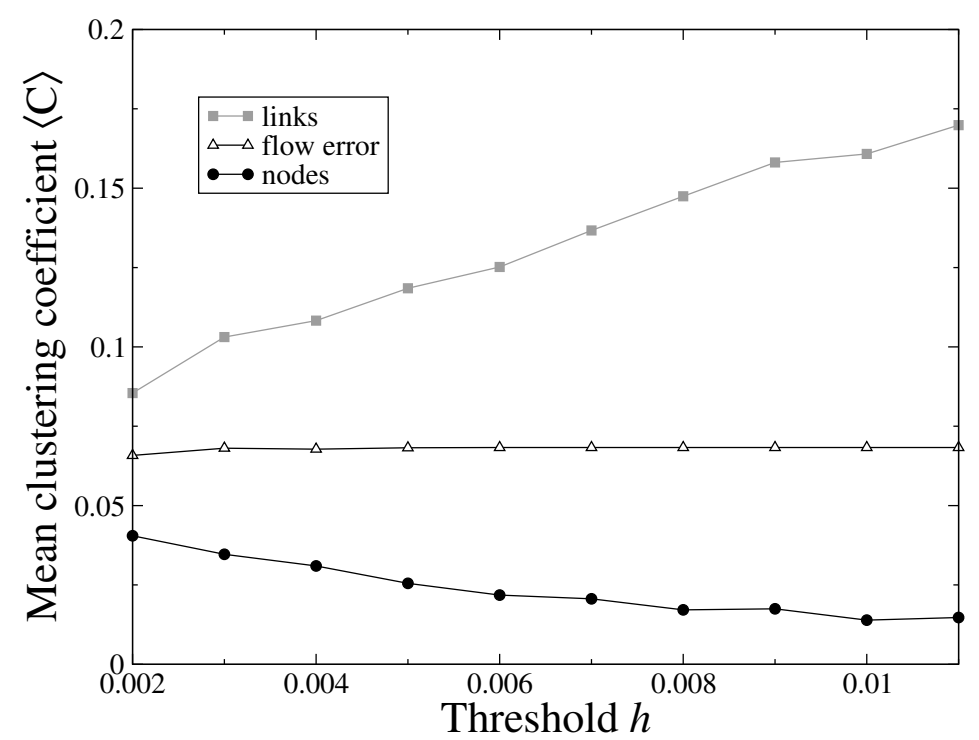

Figure 4.9: Average clustering coefficients of ensembles of the networks, optimized against node and link removals and only by flow error, as a function of the threshold used during the optimization.

\section{Average path length between input and output nodes}

An important structural propertiy of the considered pipeline networks is the average path length $(A P L)$ between the input and output nodes. For each input node, the shortest paths, connecting it to each of the output nodes, can be determined ${ }^{1}$. The average path length of a network is the average value of the lengths of all of these paths. The mean $A P L$ of an ensemble is obtained by additional averaging over all networks in the ensemble.

Figure 4.10 shows the mean average path length between input and output nodes for ensembles of networks optimized using different criteria and thresholds. Netwoks, optimized for link robustness, present a larger value of $A P L$ than the networks obtained by using two other criteria. On the other hand, networks optimized for node robustness have shorter $A P L$ than the networks optimized only by flow error.

This result may look unexpected, because the networks optimized for link robustness have larger connectivities and, therefore, communication between the nodes should be better and the paths should be shorter in this case. The observed increases in APL come probably from the fact that the output nodes, which do not need to be activated according to the target output pattern, may still be activated generating some acceptable errors. To make such errors small, the input fluxes should undergo many divisions, before they reach in small fractions these incorrectly activated output nodes. This strategy allows to increase the number of connections, and this increase produces more robust networks,

\footnotetext{
${ }^{1}$ Formally, in the graph theory, if two nodes are not connected, the distance is infinite. With this definition and since many pairs of input and output nodes are often not connected, the $A P L$ would be always infinite. Therefore, we consider here only the paths which really exist in the network.
} 


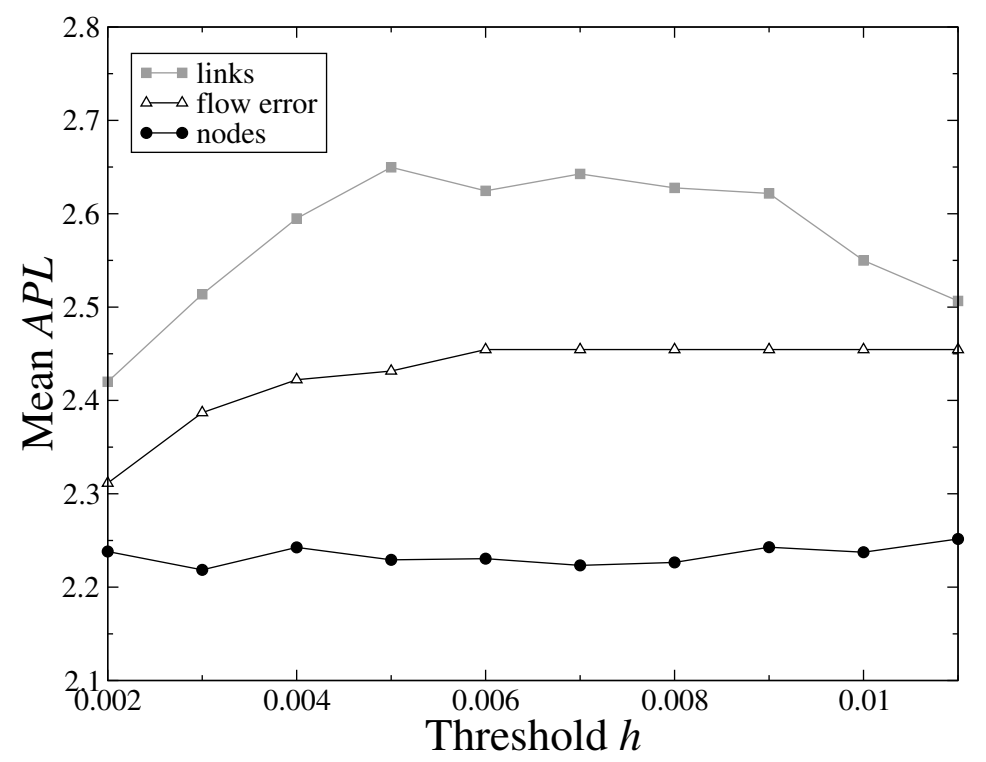

Figure 4.10: Mean average path lengths $(A P L)$ between input and output nodes in ensembles of networks optimized against node and link removals and only by flow error, as a function of the threshold used during the optimization.

since the probability of eliminate an useful connection becomes smaller.

The situation is different for the networks optimized for node robustness, where there are less steps between the input and output nodes. In this case, the input nodes do not produce the activation of incorrect output nodes, and the input unit flux must not be divided many times.

\section{Sizes of activated subnetworks}

When flux is applied to an input node, it goes only through a subset of middle nodes until it reaches the outputs. The average size of an activated subnetwork is an important property of the considered model. It is computed as the mean number of middle nodes in subnetworks corresponding to activation of different input nodes. Figure 4.11 shows average subnetwork sizes for ensembles of networks, optimized using different criteria, as a function of the optimization threshold.

In agreement with the increases in the connectivity and clustering coefficient, the networks optimized for link robustness also have subnetworks with the sizes larger than those of the networks optimized only by flow error and for node robustness. The difference in the sizes grows with the threshold. Since our networks have $M=20$ middle nodes, about half of all middle nodes becomes activated to produce a response at $h=0.007$, where the optimization is most efficient. This indicates that strong overlaps between subnetworks are then present.

Networks, optimized for node robustness, have smaller activated subnetworks than 


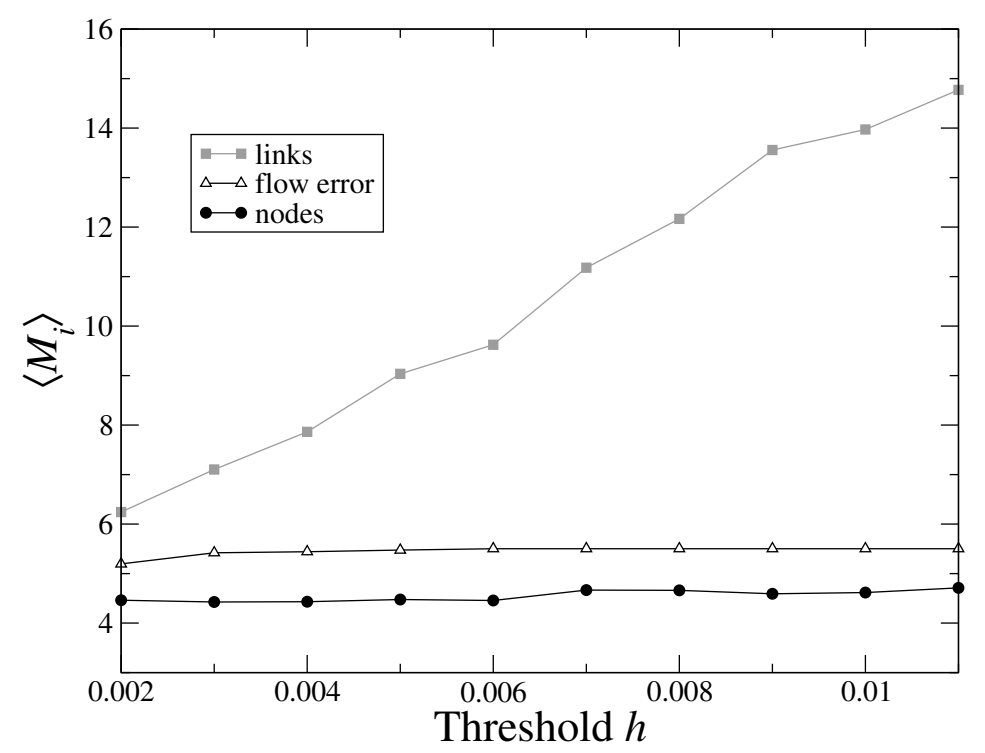

Figure 4.11: Mean values of the number of middle nodes in the subnetworks activated by the different input nodes, versus the threshold used during the optimizations.

the networks, optimized only by flow error. In such networks, each input node tries to activate the smallest subnetwork possible. Because we have $N_{i n}=8$ input nodes, overlaps between subnetworks are however present even in this case.

\subsubsection{Motif distributions of robust networks}

Next, we study motif distributions of robust networks which were optimized by using different criteria. Figure 4.12 presents the mean normalized Z score of ensembles of networks optimized against node (left) and link (right) removals. The optimizations were performed which different thresholds. Two clearly different motif distributions are found, independent of the threshold used. Statistical dispersion is larger in the ensembles optimized with small thresholds. This may be because the robustness optimization is less efficient for the smaller thresholds and, therefore, networks with different properties are mixed in the ensemble. Moreover, the number of functional networks after the optimization may be significantly less than 100 in the ensembles.

Figure 4.13 shows the motif distributions of the networks robust against link (Fig. 4.13a) and node (Fig. 4.13b) deletions, optimized using $h=0.007$. For comparison, we also show here motif distributions of different real networks. The motif distribution of the networks robust against link removals (Fig. 4.13a) shows a striking agreement with the respectives diagrams for biological signal transduction networks and the genetic developmental network of Drosophila, while the motif distribution of the model networks robust against node removals resembles that of the linguistic networks in the fourth superfamily (Fig. 4.13b). 


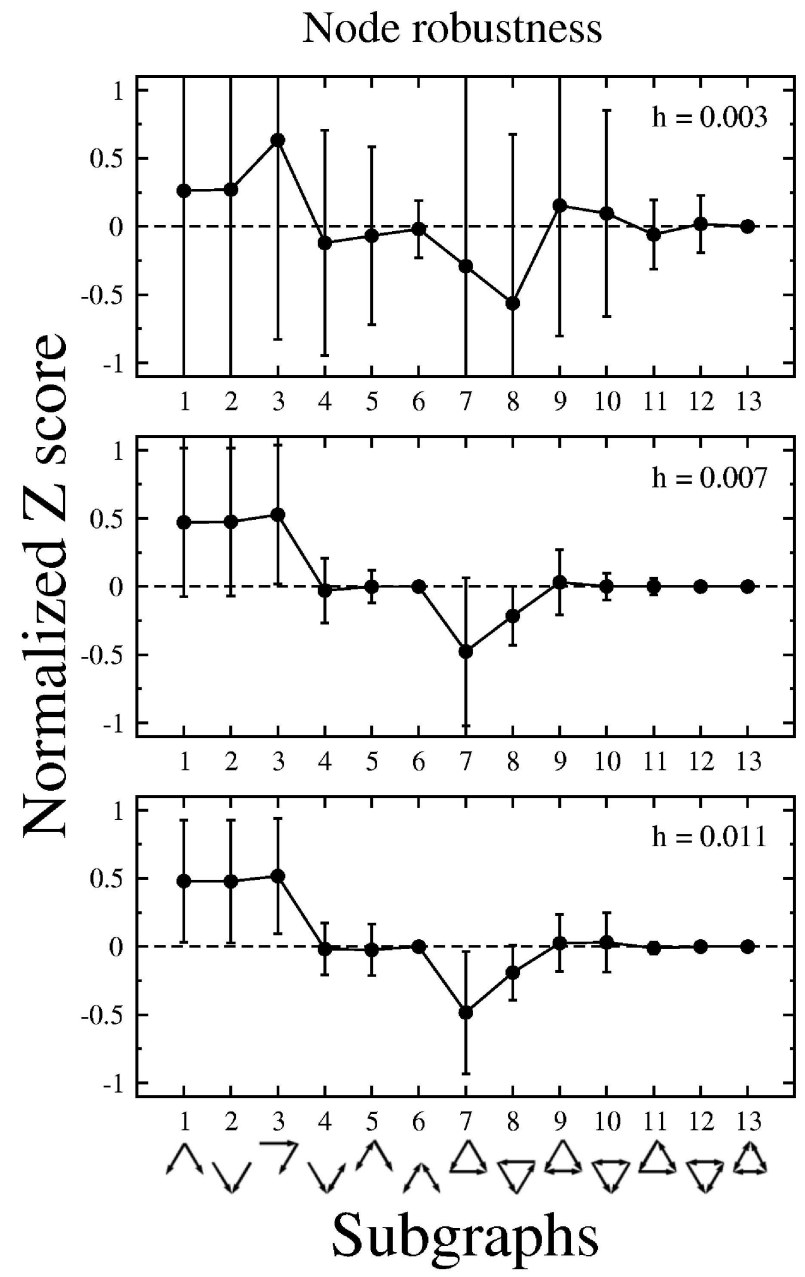

Link robustness
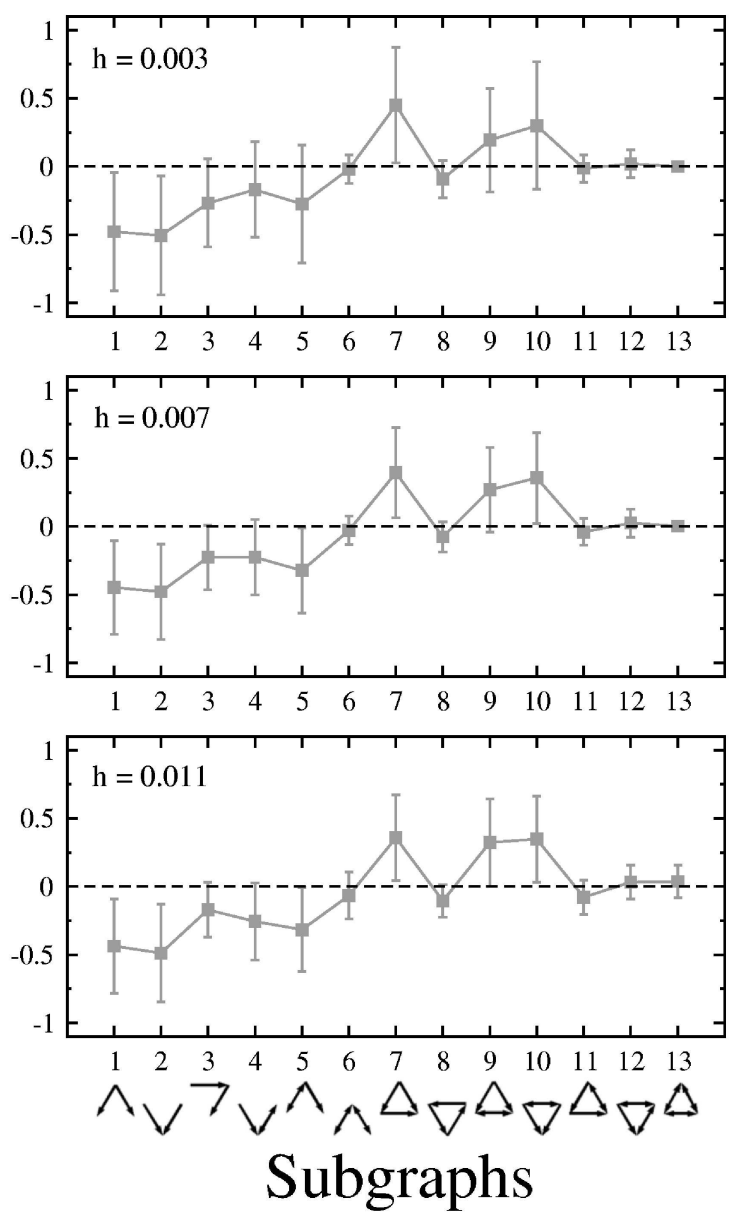

Figure 4.12: Motif distributions of networks robust against removal of nodes (left, black circles) and links (right, gray squares). The optimizations were performed using different thresholds. Bars show statistical dispertion of data.

Proceeding from this analysis, we suggest that the principal role in determining the characteristic motif distribution in the second superfamily is played by the condition of robustness against link removals, imposed by biological evolution. Signal transduction networks and genetic developmental networks of multicellular organisms may have evolved to become robust against random disruption of interactions between proteins, rather than the removal of entire proteins (nodes) from these networks. In this respect, we note that a genetic point mutation modifies only one amino acid in the protein chain and this would typically lead only to a minor change in the folded protein conformation. Therefore, it can be expected that, after such a mutation, a protein would loose only some its interactions with other proteins in the network, corresponding to breaking of individual links in the model. Moreover, the neural system of $C$. elegans may have evolved to become robust against breaking of synaptic connections, rather than against the death of whole neural 

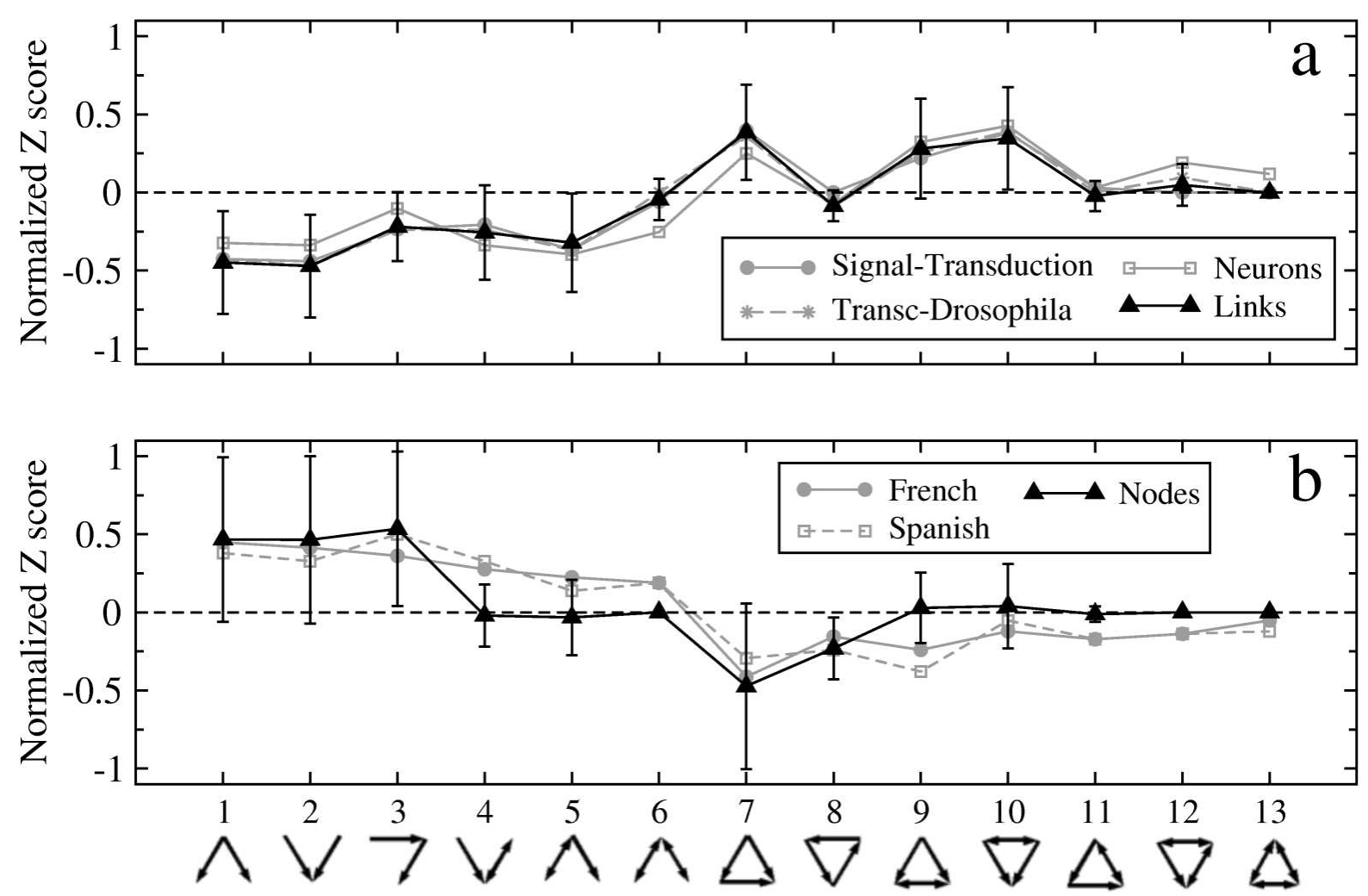

Subgraphs

Figure 4.13: Motif distributions of networks robust against removal of (a) links (triangles) and (b) nodes (triangles). The optimization was performed using $h=$ 0.007. Bars show statistical dispertion of data. For comparison, the data [46] for (a) signal transduction networks, developmental genetic transcription networks in drosophila, neural system of $C$. elegans and (b) two linguistic networks are included (gray curves).

cells.

The spectacular agreement between motif distributions of real biological information processing networks and model networks, optimized to be robust against link removals, cannot be explained by purely qualitative arguments. It may indicate the existence of a general statistical universality class of such networks. The considered minimalistic model neglects many details of actual signal transduction and genetic regulation processes. Nonetheless, it is apparently able to correctly reproduce major structural properties of networks in the second superfamily. 


\section{Chapter 5}

\section{Functional networks robust against noise}

In this chapter we consider topological noise which affects the structure of networks by changing weights of their connections. In constrast to random deletion of single nodes or links, such noise represents distributed weak damage. First, robustness against this kind of damage is defined. Then, an evolutionary optimization algorithm is applied to construct networks robust against noise. Statistical properties of the networks robust against noise are considered and compared with the properites of the networks optimized only by flow error. Finally, we compute motif distrubutions of these robust networks and compare them with the motif distributions of networks robust against local damages.

\subsection{Noise in flow distribution networks}

Noise in real biochemical systems is because concentrations of chemical species are fluctuating (internal noise) and because parameters, such as temperature, can irregularly vary with time (external noise). To study the effects of such dynamical noise, a model with temporal evolution is needed. In our considered pipeline network model no dynamics is present and this kind of noise cannot be studied. However, we can still have some static or "frozen" noise, representing distributed random weak damage of the network structure. We will call it the topological noise.

\subsubsection{Definition of topological noise}

According to the definition of the pipeline model in Chapter 2, the total input flux in each node is split in equal parts among its outgoing connections. This means that the outgoing connections are considered as having the same weights. Topological noise will change these weights, diminishing the weights of existing connections and creating new weak connections if they were absent. The output fluxes of a node are then divided 
proportionally to the weights of the outgoing connections, modified by noise.

A weight $W_{i j}$ of a connection is changed by noise as

$$
W_{i j}=\left\{\begin{array}{c}
1-S \xi_{i j}, \text { if } A_{i j}=1 \\
S \xi_{i j}, \text { if } A_{i j}=0
\end{array}\right.
$$

where $S$ is the noise intensity, $\mathbb{A}$ is the adjacency matrix and $\xi_{i j}$ is a random variable with uniform distribution in the interval between zero and one. It is important to note that all weights of the network are affected simultaneously by the noise, representing a particular realization of the set of random variables $\xi_{i j}$.

With this noise, the input flow to each node [equation (2.3)] must be modified. Now we have

$$
x_{i}=\sum_{j=1}^{N} \frac{W_{i j}}{\sum_{k=1}^{N} W_{k j}} x_{k}
$$

When the noise intensity $S$ is zero, $W=\mathbb{A}$ and this equation is reduced to equation (2.3), since $\sum_{k=1}^{N} W_{k j}$ is just the outgoing degree of the node $j$.

Applying noise to a network $G$ with flow error $\epsilon$ with respect to some target output pattern $\mathbb{Q}^{0}$, we obtain a new network $G(\xi)$ with a new output pattern $\mathbb{Q}(\xi)$ and a new flow error $\epsilon(\xi)$, which is usually larger than $\epsilon$. In Fig 5.1 we construct distributions of the flow errors $\epsilon(\xi)$ of networks obtained after the application of noise to a network $\bar{G}$, optimized only by flow error (the flow of the network $G$ itself is $\bar{\epsilon}=0.0038$ ).

Each distribution corresponds to the normalized histogram of $N_{S}=300$ noise realizations, for each noise intensity $S$. The mean flow error increases with the noise and the distributions also become more broad.

\subsubsection{Definition of noise robustness}

Similar to the case of local damages, we introduce noise robustness $\rho_{s}$ of a network $G$ as the ratio between the number of non-abortive noise realizations $(\epsilon(\xi)<h)$ and the total number of them. Formally, the noise robustness is the mean value taken over all possible realizations

$$
\rho_{s}(G)=\langle\Theta(h-\epsilon(\xi))\rangle_{\xi}
$$

To compute it, we choose an ensemble with $N_{S}$ noise realizations and determine

$$
\rho_{s}(G)=\frac{1}{N_{S}} \sum_{G(\xi) \in S} \Theta(h-\epsilon(\xi))
$$

This ensemble is similar to the damage shells for local damages (deletion of a node or of a link).

Topological noise has two principal effects. It weakens existing connections $\left(A_{i j}=1\right)$ and generates many new weak ones. The first effect produces loss of flow in the paths 


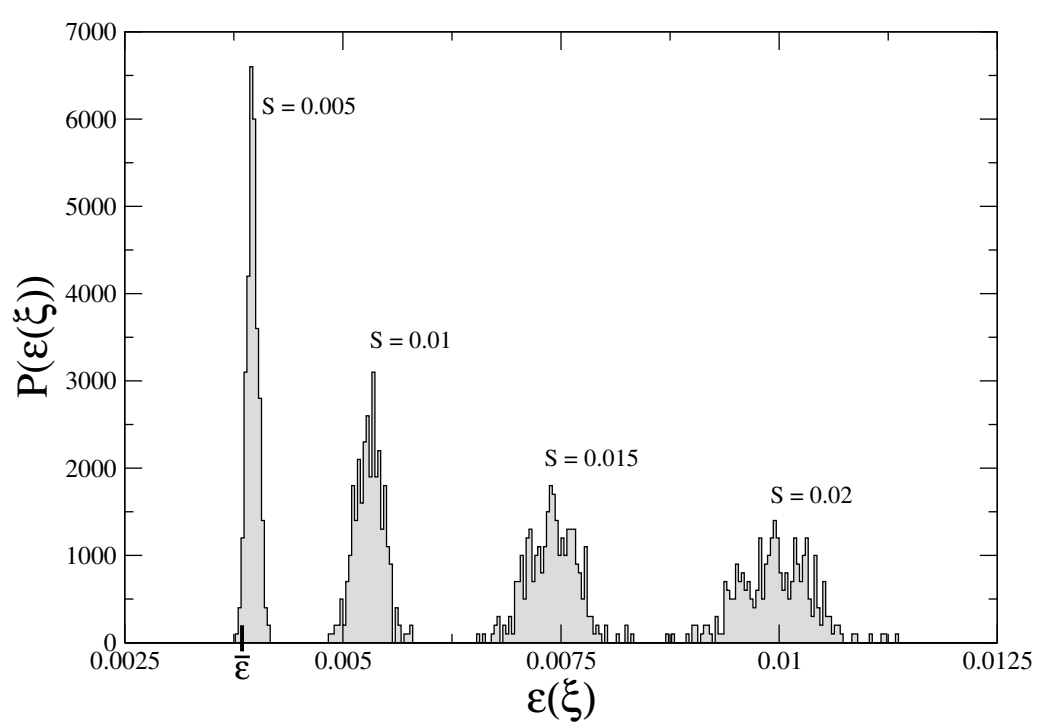

Figure 5.1: Distributions of flow errors for the same network, when noises of different intensity $S$ are applied. To compute each distribution, 300 independent noise realizations are taken. The networks have $N=36$, with $N_{\text {in }}=N_{\text {out }}=8, M=20$ and $K=4$.

between the input and output nodes. In consequence, the amount of flow arriving to the nodes which have to be activated $\left(Q_{i j}^{0} \neq 0\right)$ decreases and the flow error grows. On the other hand, new weak connections distribute the lost flows almost equally among all ouput nodes, generating a random background of weak flows to the outputs. This effect also increases the flow error, because many incorrect output nodes (where $\mathbb{Q}_{i j}$ must have been zero) become weakly activated. Both effects significantly depend on the networks size, since the number of new possible weak connections grows with the number of middle nodes.

Figure 5.2 illustrates this behaviour for the networks optimized only by flow error. Figure 5.2a shows the mean flow errors $\langle\epsilon\rangle$ of these networks and their mean flow errors $\langle\epsilon(\xi)\rangle$ after the application of noise. While $\langle\epsilon\rangle$ decreases with $M$, the mean error $\langle\epsilon(\xi)\rangle$ of the networks with noise is slowly growing with the number of middle nodes $M$, because more potential connections become then possible.

Figure $5.2 \mathrm{~b}$ shows mean noise robustness $\left\langle\rho_{f}^{s}\right\rangle$ of these networks as function of $M$. Networks with a few middle nodes are not robust since they have high flow errors. Large networks are also vulnerable against noise. There exists an interval of network sizes where (for this noise intensity) the networks optimized by flow error are also relatively well robust against noise.

We can roughly estimate such effects as follows. According to (5.1), mean values of the weights with noise are

$$
\bar{W}_{i j}=\left\{\begin{array}{c}
1-\frac{1}{2} S, \text { if } A_{i j}=1 \\
\frac{1}{2} S, \text { if } A_{i j}=0
\end{array}\right.
$$



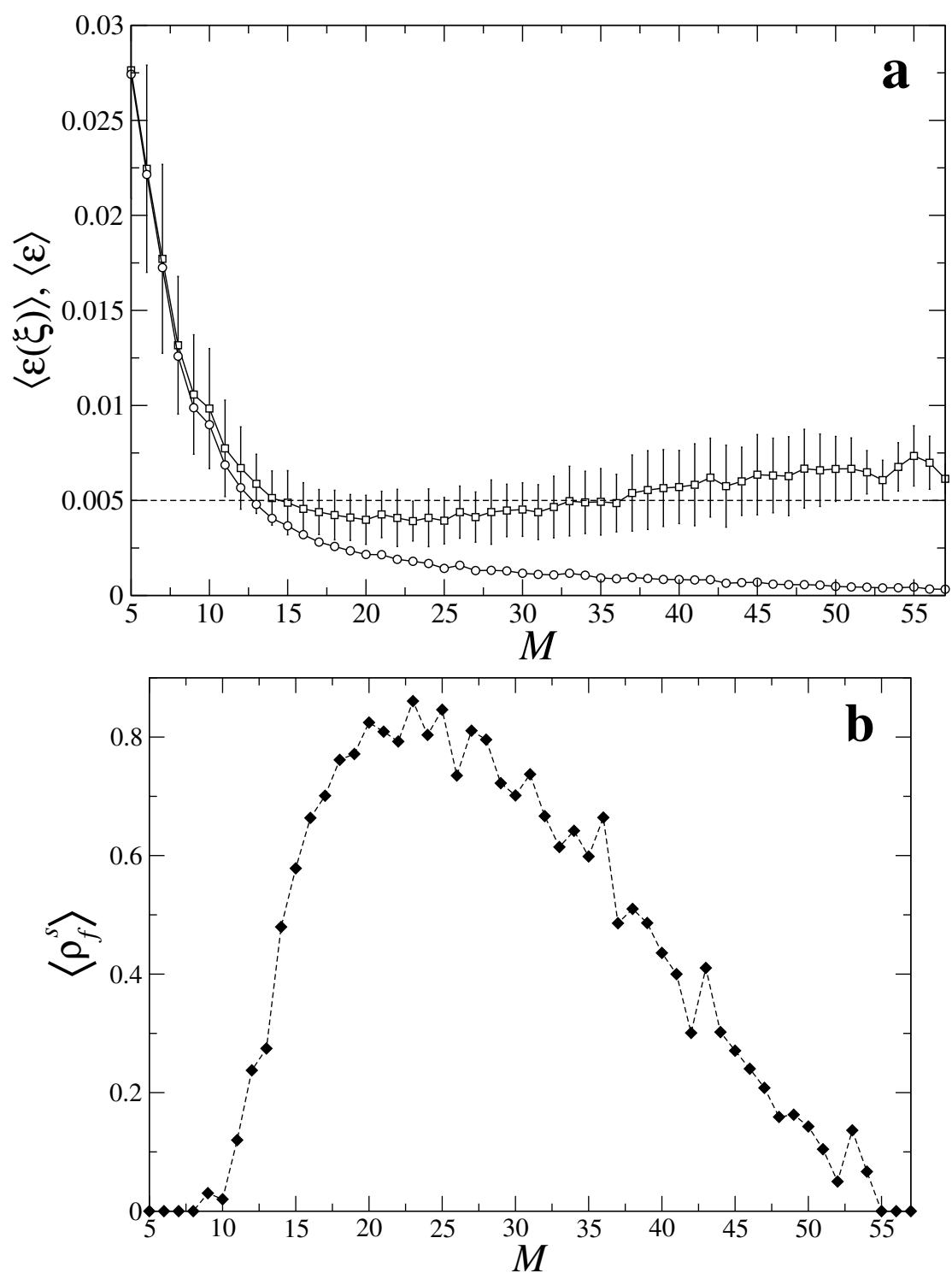

Figure 5.2: (a) Mean flow errors $\langle\epsilon\rangle$ of ensembles of networks, optimized only by flow error, and their mean flow errors $\langle\epsilon(\xi)\rangle$, with the noise of intensity $S=0.01$ applied, for different numbers of middle nodes. (b) Mean noise robustness $\left\langle\rho_{f}^{s}\right\rangle$ of these networks. The networks have $N_{\text {in }}=8$ and $N_{\text {out }}=8$, and their target output patterns have $K=4$. Each ensemble includes 100 networks. For each network, $N_{S}=300$ independent noise realizations are taken.

The mean output degree of a node is $D_{\text {out }}$ and the total number of possible outgoing connections is $U$ ( $U=M$ for input nodes, and $U=M-1+N_{\text {out }}$ for middle nodes). The mean normalization factor $\sum_{k=1}^{N} W_{k j}$ is equal to a sum of the weights of preexisting connections $\left(1-\frac{1}{2} S\right) D_{\text {out }}$ and of the new weak connections generated by the noise incorporation $\frac{1}{2} S\left(U-D_{\text {out }}\right)$. 
The total mean flux $f$ through preexisting connections is

$$
f=\frac{D_{\text {out }}\left(1-\frac{1}{2} S\right)}{D_{\text {out }}+\frac{1}{2} S\left(U-2 D_{\text {out }}\right)}
$$

and the mean flow $\bar{f}=1-f$ lost through the new connections generated by the noise is

$$
\bar{f}=\frac{S\left(U-D_{\text {out }}\right)}{2 D_{\text {out }}-S\left(U-2 D_{\text {out }}\right)}
$$

Note that the mean lost flow increases with $S$ and with the size of the network $U$. Therefore, we can expect that the mean flow error of the networks with noise would indeed be higher for the larger networks.

\subsection{Evolutionary design of networks robust against noise}

Functional networks robust against noise can be constructed using the same optimization method as in the previous chapter.

\subsubsection{Optimization algorithm}

We apply the same kind of Metropolis algorithm as used before to construct functional networks robust against local damages. However, we want to increase robustness against noise, calculated using equation (5.4) and taking an ensemble of $N_{S}$ noise realizations. The number $N_{S}$ is related to the size of the network which is optimized. We take 10 noise realizations by each middle node of the network, so that the total number of noise realizations is $N_{S}=10 M$.

In each iteration, a network $G$ with flow error $\epsilon$ and noise robustness $\rho_{s}$, which is being optimized, is mutated generating a new network $G^{\prime}$ with flow error $\epsilon^{\prime}$ and noise robustness $\rho_{s}^{\prime}$. The selection criterion depends on the value of the flow error of $G$ with respect to the threshold considered. If $\epsilon>h$, the network is not functional and we reduce its flow error. In this case, if $\Delta \epsilon=\epsilon^{\prime}-\epsilon<0$, we accept the mutation, and if $\Delta \epsilon>0$, we accept the mutation with probability $\exp \left(-\Delta \epsilon / \epsilon \sigma_{E}\right)$. When $\epsilon<h$, the network is already functional and we increase its robustness. If $\Delta \rho_{s}=\rho_{s}-\rho_{s}^{\prime}<0$, we accept the mutation. If $\Delta \rho_{s}>0$, we accept the mutation with probability $\exp \left(-\Delta \rho_{s} /\left(1-\rho_{s}\right) \sigma_{R}\right.$.

The optimization process is performed for a fixed number of iterations or until the network reaches the maximum possible value of the noise robustness $\left(\rho_{s}=1\right)$. In each evolution, we use a random Erdös-Rényi network with random connectivity $p$ as the initial condition and we generate a new random output pattern $\mathbb{Q}^{0}$. We use the path mutation scheme for evolutionary mutations, adding or deleting random paths between input and output nodes (see Fig. 3.1). 
The number of middle nodes is fixed during the evolution and isolated nodes are deleted from the network when optimization is ended. To evaluate noise robustness during the evolution process, we take into account all nodes, even if they are disconnected.

\subsubsection{Example of construction of a robust network}

Figure 5.3 shows an example of the construction of a functional network robust against noise. In Fig. 5.3a, the obtained network is displayed. Figure 5.3b shows the behavior of the flow error $\epsilon$ and the noise robustness $\rho_{s}$ during the evolution.
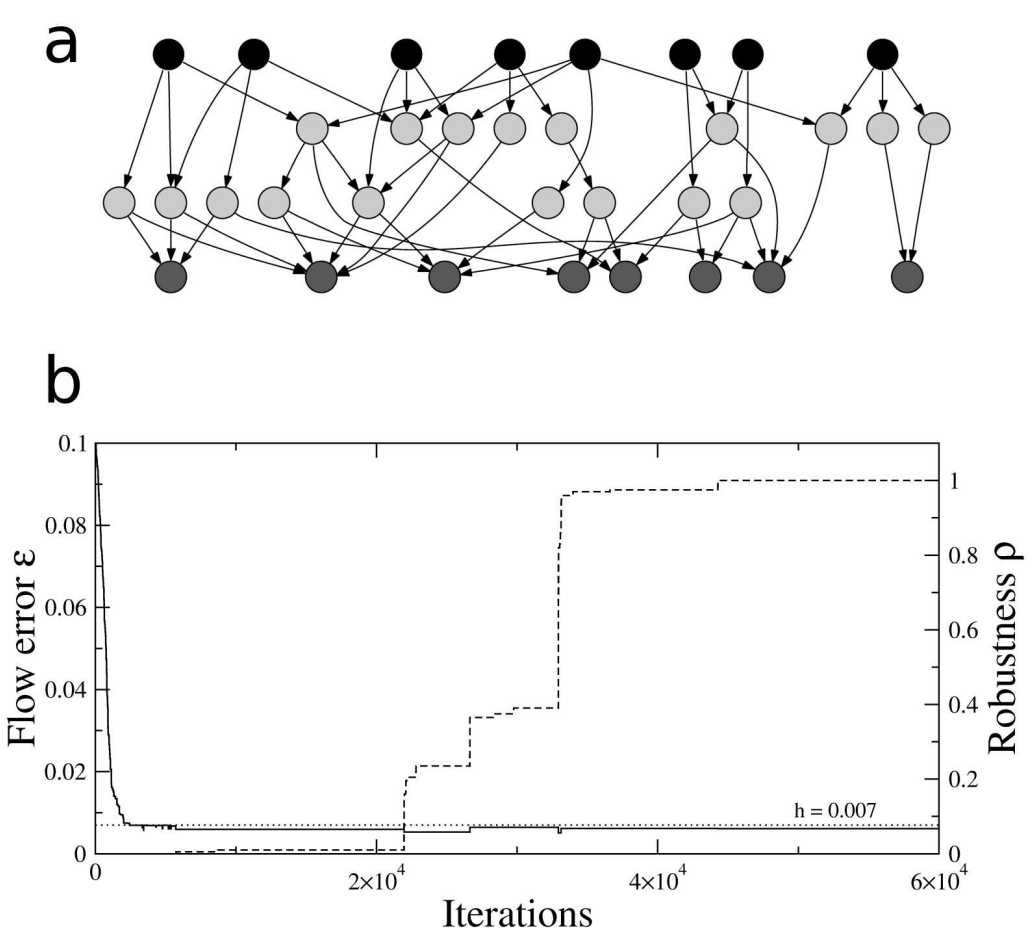

Figure 5.3: (a) The network obtained by noise robustness optimization and (b) the evolution of the flow error (solid line) and robustness (dash line). Here, $h=0.007$, $S=0.02$ and $\sigma=10^{-4}$.

After the selection has been switched from the flow error to robustness, robustness has rapidly increased and has reached the value of unity in the considered case. The flow error remains under the threshold $h$, as required by the optimization method.

\subsubsection{Temperature parameter}

We have determined the optimal temperature parameter $\sigma_{R}$ used in the maximization of noise robustness. Since the constructed networks mainly have robustness near one 
or zero, a different property is chosen for the study. Figure 5.4 shows the number of successful constructions (with the final network having robustness different from zero) in the ensembles of 100 independent evolutions as a function of the temperature parameter $\sigma_{R}$.

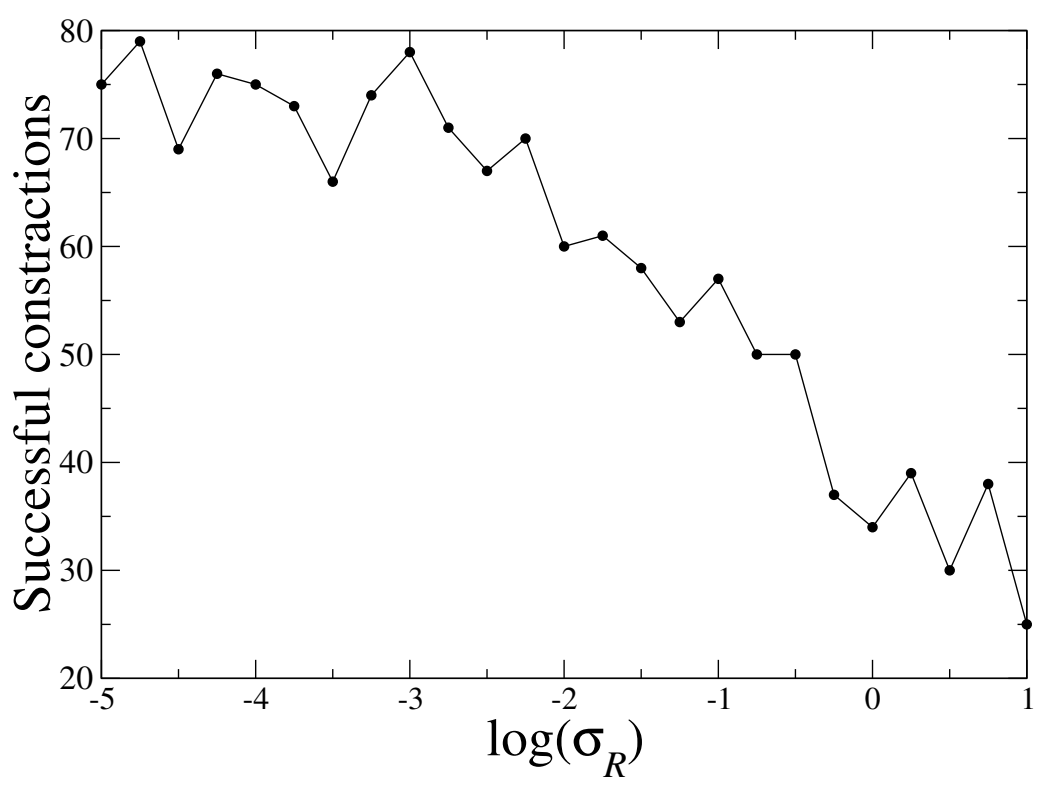

Figure 5.4: Number of successful constructions versus $\log \left(\sigma_{R}\right)$. Each ensemble has 100 networks. The parameters are $N_{\text {in }}=8, M=20, N_{\text {out }}=8, K=4, h=0.007$, $S=0.02, \sigma_{E}=10^{-4} ; 1 \times 10^{5}$ iterations are performed.

We see that the rate of successful constructions increases as $\sigma_{R}$ gets smaller. Therefore, below in this chapter, small values $\sigma=\sigma_{E}=\sigma_{R}=10^{-4}$ of these temperatures parameters are chosen.

\subsection{Statistical properties of designed networks}

To analyze statistics of evolutionary optimization against noise and to consider structural properties of designed networks, ensembles of 100 networks for each of noise intensity and each threshold are considered. The sizes of the networks are $N=36$, with $N_{\text {in }}=8$, $M=20, N_{\text {out }}=8$, and the target output patterns have $K=4$. The number of iterations used in the simulations is $10^{5}$, and the temperature parameters are $\sigma=10^{-4}$. As before, the target output patterns are randomly generated for each evolution. The initial conditions are randomly generated networks with arbitrary connectivity $p$. 


\subsubsection{Flow error of designed networks}

In Fig. 5.1, we have seen that the mean value and the statistical dispersion of flow errors after application of noise to the networks, designed by optimization only for flow error, are gradually increased. Now, we want to study distributions of flow errors in the networks specially optimized for robustness against noise.

Figure 5.5 shows mean flow errors of ensembles of networks optimized for noise robustness (filled symbols) and only by flow error (empty symbols), as a function of noise intensity. Flow errors of robust networks (filled circles) remain close to the threshold in

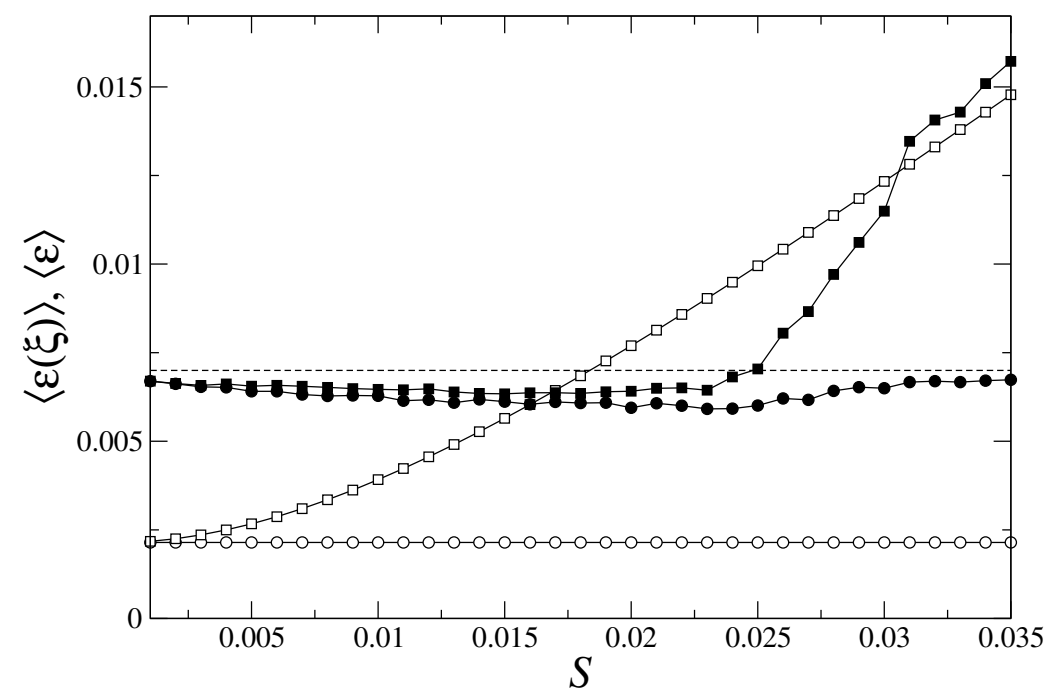

Figure 5.5: Mean flow errors $\langle\epsilon\rangle$ of the optimized networks (circles), and mean flow errors $\langle\epsilon(\xi)\rangle$ of the noise realization networks (squares). The filled symbols correspond to networks optimized by noise robustness and the empty ones are of networks optimized only by flow error. The threshold used during the optimizations was $h=0.007$.

the whole range of noise intensities considered.

Figure 5.5 also shows mean flow errors $\langle\epsilon(\xi)\rangle$ of the networks with noise applied, for the networks optimized for noise robustness (filled squares) and only by flow error (empty squares). In the networks, optimized only by flow error, these mean values grow with the noise intensity. In the networks, optimized against noise, the behaviour is very different. In this case, mean errors $\langle\epsilon(\xi)\rangle$ remain below the threshold in a wide range of noise intensities (for $S<0.025$ ). For stronger noise, the optimizations becomes less successful, and the errors grow, approaching the values for the networks optimized only by flow error.

Figure 5.6 shows four characteristic distributions of flow errors after the application of noise. A remarkable difference with the distributions of Fig. 5.1 for the networks optimized only by flow error can be seen. The error distributions in Fig. 5.1 have roughly Gaussian shapes, with the width growing with the noise intensity. In contrast to this, the distributions in Fig. 5.6 are clearly asymmetric. The fraction of noise realizations, 

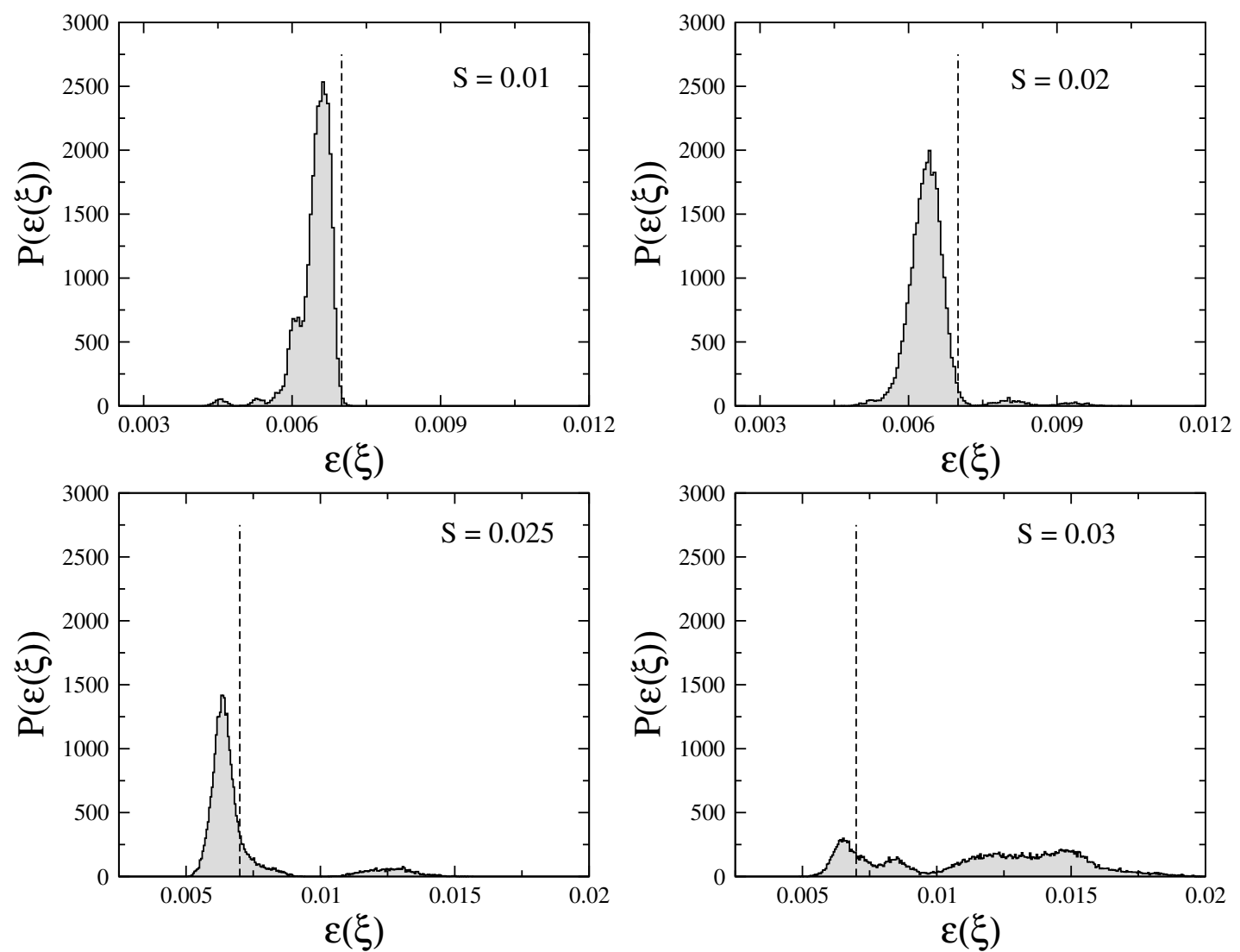

Figure 5.6: Flow errors of networks after application of various noise realizations for the networks optimized by noise robustness. The threshold is $h=0.007$.

leading to errors exceeding the threshold, is largely reduced. Most of the realizations have flow errors just below the tolerance threshold $h$. As the noise intensity $S$ is increased, more noise realizations yield networks with $\epsilon(\xi)>h$ and a tail spreading towards large error values is formed. At $S=0.03$, the optimization against noise is effectively breaking down, with the majority of noise realizations having large flow errors. But even in this case, the distribution of flow errors is strongly affected by the optimization process.

\subsubsection{Robustness of designed networks}

The importand changes in the flow error distributions of networks optimized for noise robustness, which we have just seen, suggest that noise robustness of these networks should be higher. Figure 5.7 shows the mean noise robustness of the networks optimized only by flow error $\left(\left\langle\rho_{f}^{s}\right\rangle\right)$ and for noise robustness $\left(\left\langle\rho_{s}^{s}\right\rangle\right)$, for different noise intensities. Inside an interval of noise intensities $(0.0125<S<0.03)$, the networks optimized for noise have much better robustness than the networks optimized only for their function (i. e., by flow errors).

Figure 5.8 shows differences $\left\langle\rho_{s}^{s}\right\rangle-\left\langle\rho_{f}^{s}\right\rangle$ between the mean noise robustness of networks 


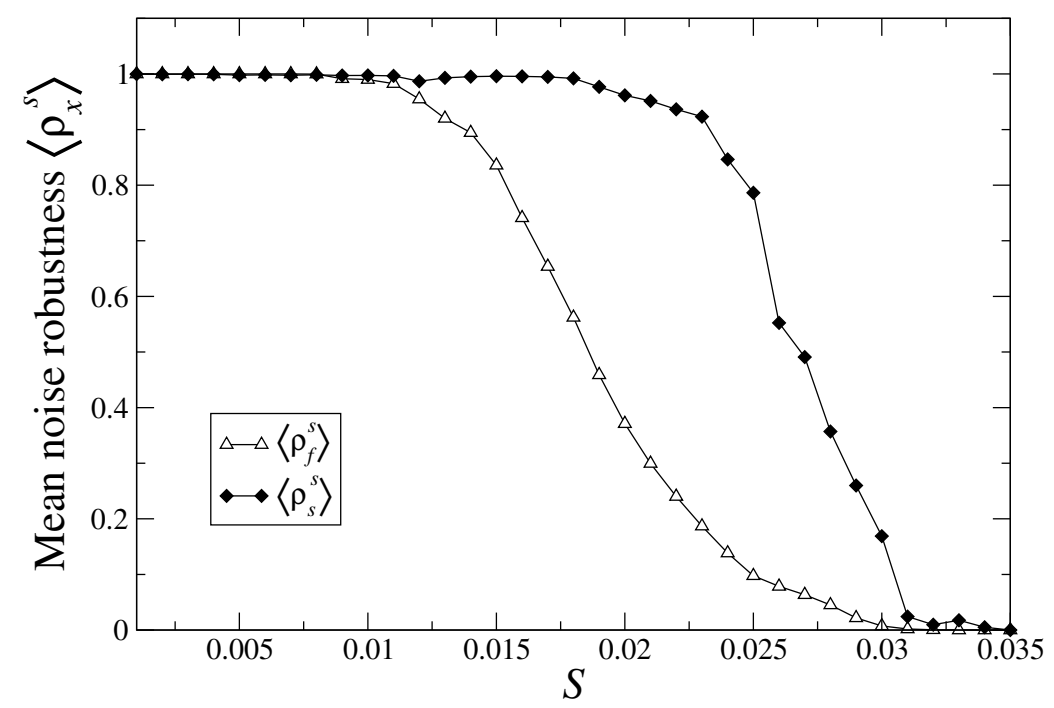

Figure 5.7: Mean noise robustness as a function of noise intensity $S$ for the ensembles of networks optimized only by flow error (empty triangles) and of the networks optimized for noise robustness (black diamonds). The threshold is $h=0.007$.

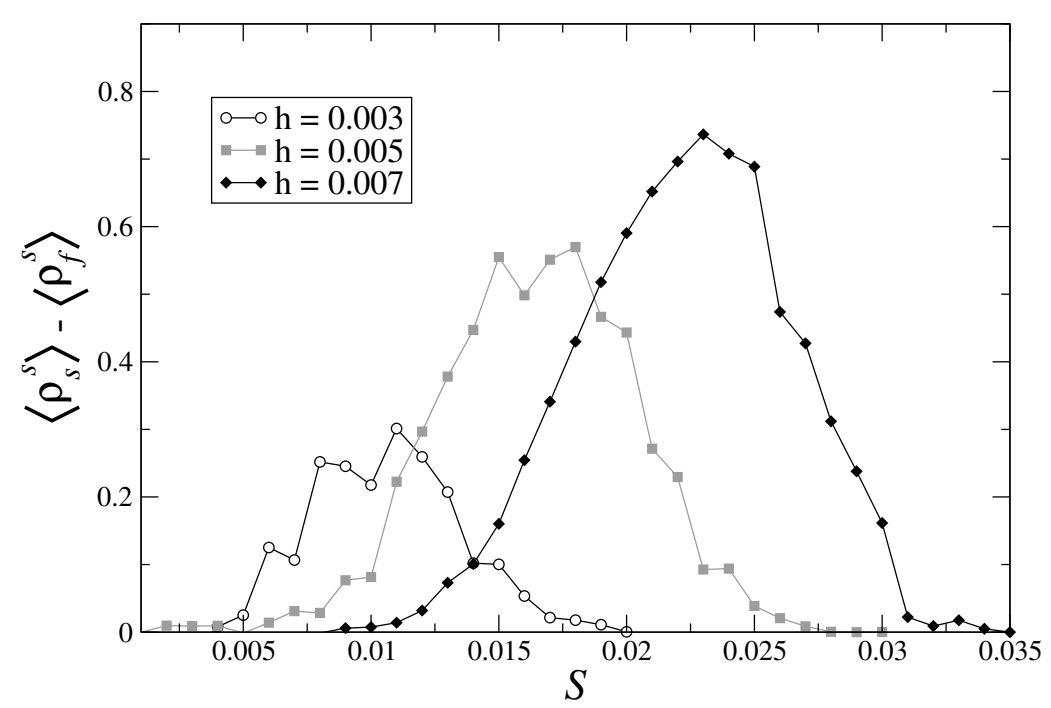

Figure 5.8: Differences between the mean noise robustness of the networks optimized for noise robustneess $\left\langle\rho_{s}^{s}\right\rangle$ and that of the networks optimized only by flow error $\left\langle\rho_{f}^{s}\right\rangle$ for different thresholds as function of the noise intensity.

optimized for noise robustenss (using different threshold values) and of the networks optimized only by flow error. Such differences characterize how much the robustness is increased after the optimization, as compared to the natural robustness of the networks optimized only by flow error. We see that the windows of noise intensity, where the optimization is successful, depend on the threshold used. The increases in the noise robustness are stronger when larger thresholds are used. Apparently, there exists a compromise be- 

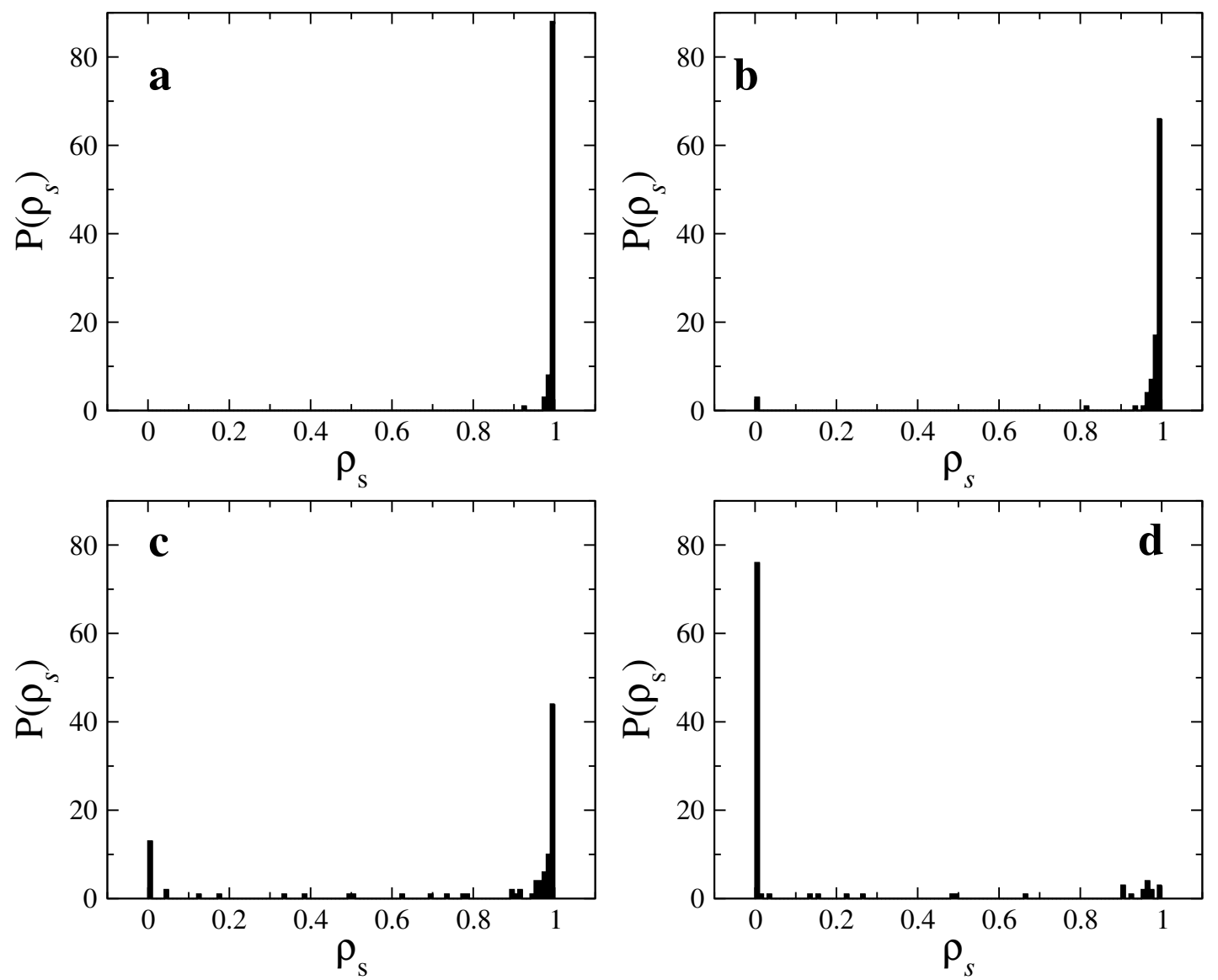

Figure 5.9: Histograms of noise robustness for several ensembles of networks optimized against noise $(h=0.007)$. The noise intensities $S$ are (a) 0.01 , (b) $0.02,(\mathrm{c})$ 0.025 and $(d) 0.03$.

tween the small flow error and the large robustness.

Finally, we present in Fig. 5.9 several histograms of the values of noise robustness the networks optimized against noise. We observe that the noise robustness of these networks takes mainly the values of zero or one, with the other values appearing much less frequently. Thus, either an evolutionary optimization has been successful and a robust network (with $\rho_{S}=1$ ) is obtained or it has failed $\left(\rho_{S}=0\right.$ ).

\subsubsection{Structural statistical properties of designed networks}

As in the previous chapters, we investigate how the requirement of noise robustness, in this case, produces changes in the architecture of the designed networks. The mean values of different statistical properties are taken for the ensembles of 100 networks, optimized against noise using different thresholds and noise of different intensities.

Figure 5.10 shows the average mean degree of the networks optimized only by flow 


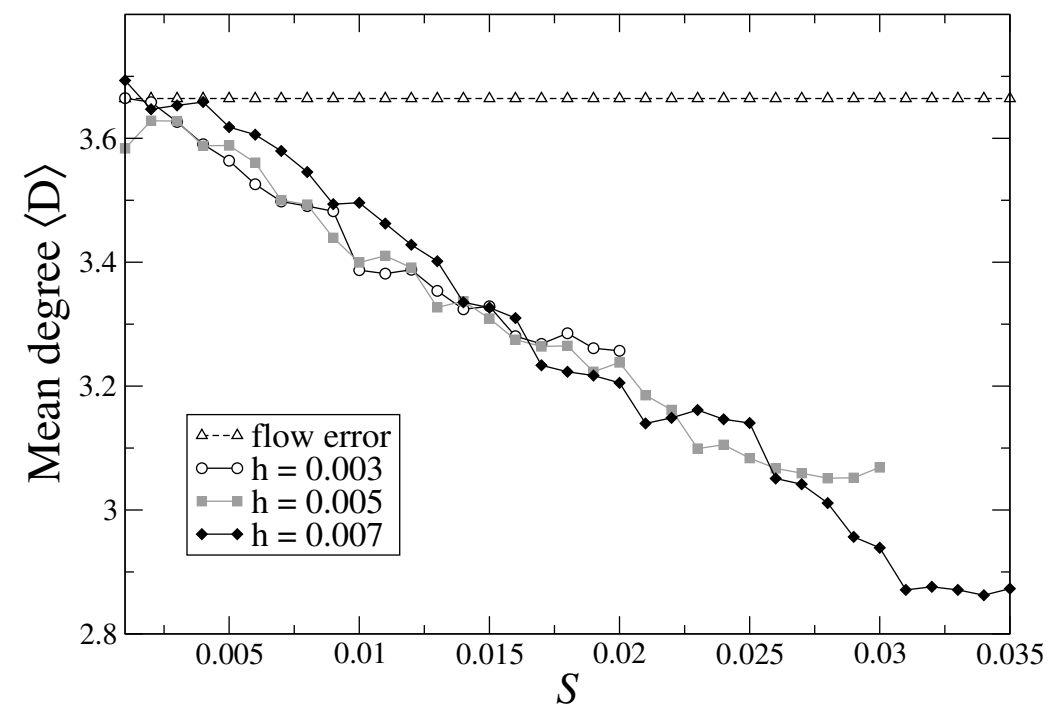

Figure 5.10: Average mean degree as a function of the noise intensity for the ensembles of networks optimized only by flow error (empty triangles) and for noise robustness, using different thresholds $h$.

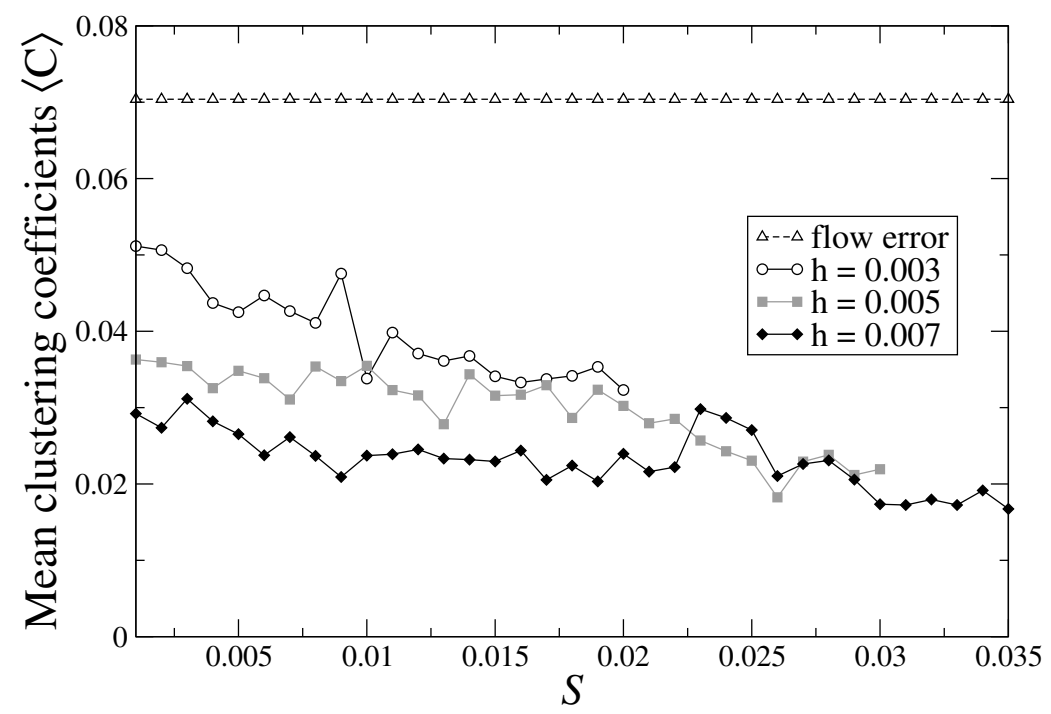

Figure 5.11: Mean clustering coefficients as a function of the noise intensity for several ensembles of networks optimized only by flow error (empty triangles) and by noise robustness, using different thresholds $h$.

error and against noise. We see that the mean degree of the networks optimized against noise is steadily decreasing with the noise intensity. This behaviour is found for different thresholds $h$. Thus, noise optimization leads to a decrease in the number of connections in the designed networks.

Figure 5.11 shows the mean clustering coefficient of the designed networks. Robust networks have smaller values of $\langle C\rangle$ in the entire considered range of noise intensities. 

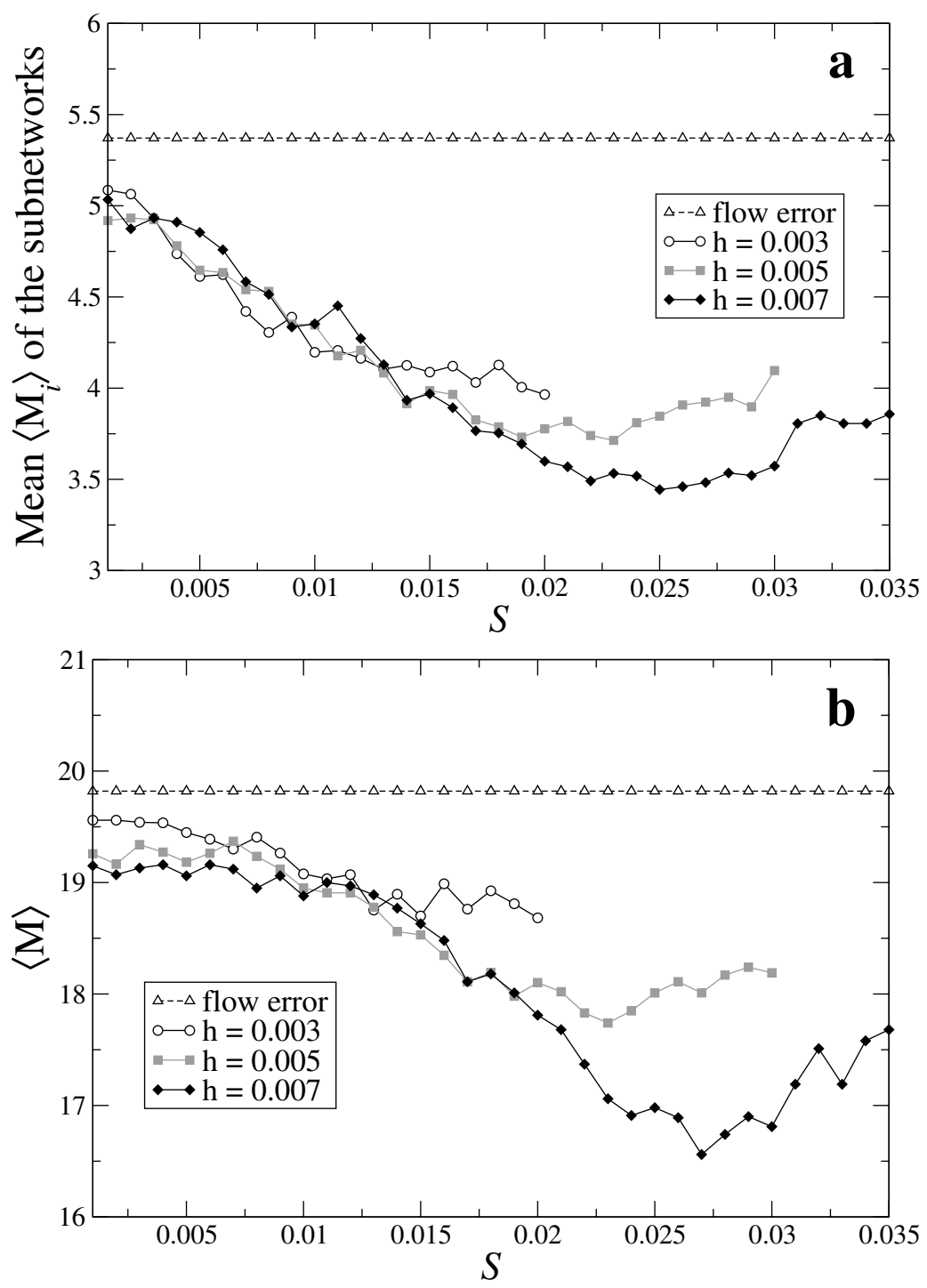

Figure 5.12: (a) Mean numbers of middle nodes in the subnetworks activated by a single input node as function of the noise intensity for the networks optimized only by flow error (empty triangles) and for noise robustness, using different threshold values. (b) Mean total numbers of the middle nodes after the elimination of isolated nodes when the simulation has ended.

In Fig. 5.12a, mean numbers of middle nodes in the activated subnetworks are shown for ensembles of networks optimized using different thresholds. We observe that the number of middle nodes in an activated subnetwork is smaller for the networks optimized against noise and the difference increases with the noise intensity. Similar behaviour is found for all three thresholds considered.

The reduction of the number of middle nodes, activated by a single input node, is because the evolution can find good solutions (with flow error smaller than the threshold) using less middle nodes, as Fig. 5.12b implies. For larger noise intensities $S$, optimization 
against noise leads to the networks where more middle nodes are isolated. Such middle nodes are not involved in the formation of outputs and are discarded in the final designed network. Again similar behaviour is observed for different thresholds.

\subsubsection{Motif distributions}

We have computed motif distributions of the networks designed to be robust against noise, for ensembles of 100 networks and using different thresholds and noise intensities.
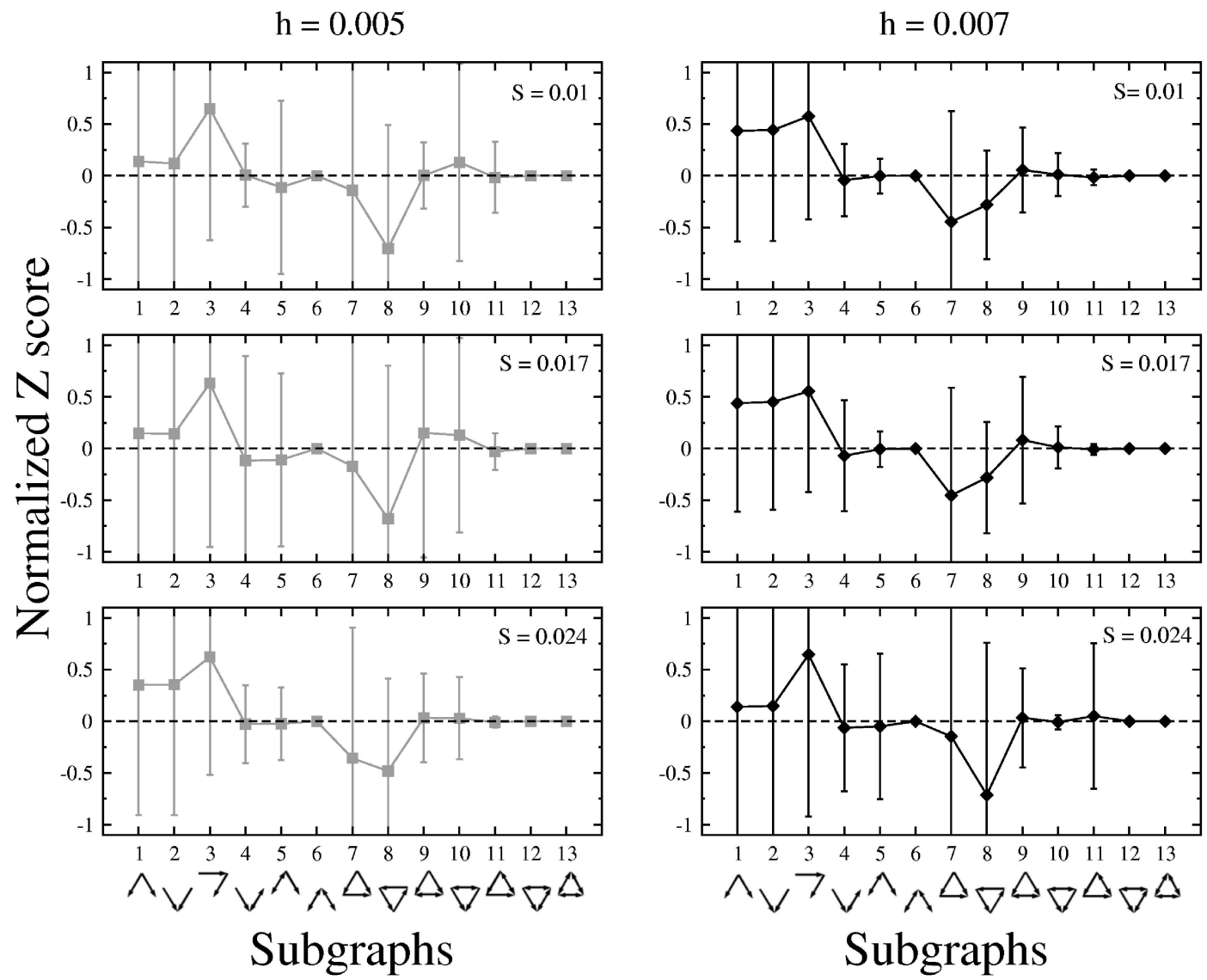

Figure 5.13: Motif distributions of networks optimized for noise robustness by different values of thresholds and noise intensities. Bars show statistical dispersion of data. The networks have $N_{\text {in }}=N_{\text {out }}=8$ and the number of middle nodes during the optimization is $M=20$. The target output patterns have $K=4$. The evolutions are terminated after $10^{5}$ iterations.

Such distributions are shown in Fig. 5.13. Similar profiles are found for all the considered cases. The strong dispersions is because the networks have small sizes and relatively few connections.

In Fig. 5.14, we compare motif distributions of the networks robust against noise 


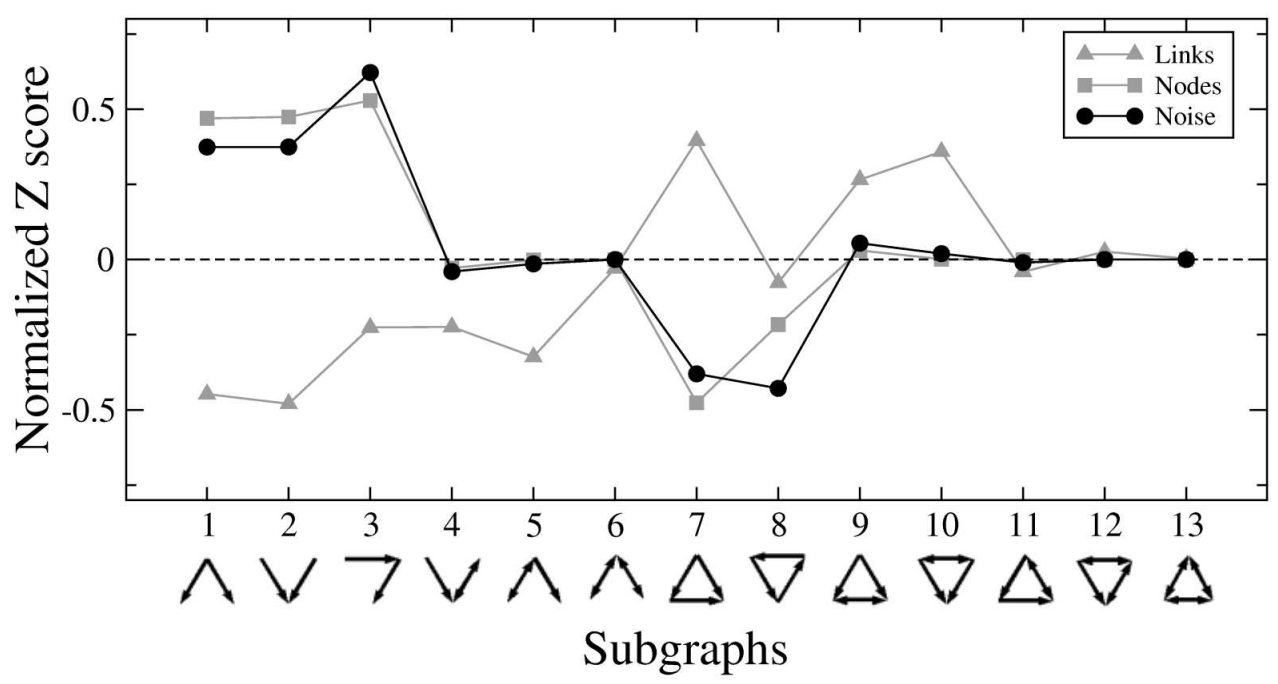

Figure 5.14: Motif distribution of the networks selected to be robust against the application of noise (bold curve, circles). The noise intensity is $S=0.02$, the tolerance threshold is $h=0.007$. For comparison, gray curves show motif distributions for the networks robust against the removal of individual nodes (squares) or links (triangles).

(optimized using $S=0.02$ and $h=0.007$ ) and of the networks optimized for node and link robustness (with the same threshold $h$ ).

It is seen that networks robust against noise have the motif distribution similar to that of the networks robust against the removal of nodes and clearly different from the motif distribution characteristic for the networks robust against the removal of links. According to our results, networks robust against (static) noise and against the removal of individual nodes belong to the fourth motif superfamily (together with the linguistic networks, see Fig 1.9), whereas the networks robust against the removal of links are in the second superfamily (together with signal transduction networks and genetic development networks of biological macroorganisms).

Analyzing motif distributions in the networks robust against noise, we have thus found that their motif distributions are different from those of the networks with the same function, but which were designed to be robust against link removals. This indicates that robustness against a particular kind of structural perturbations may play a principal role in determining motif distributions. 


\subsection{Comparison of cross-robustness of various designed networks}

There is a strong similarity between structural statistical properties of the networks optimized against noise and against the removal of a node. In both cases, the mean degrees, the clustering coefficients and the numbers of middle nodes in the subnetworks are reduced with respect to their values for the networks optimized only by flow error. Moreover, both kinds of networks show similar motif distributions. We want to discuss
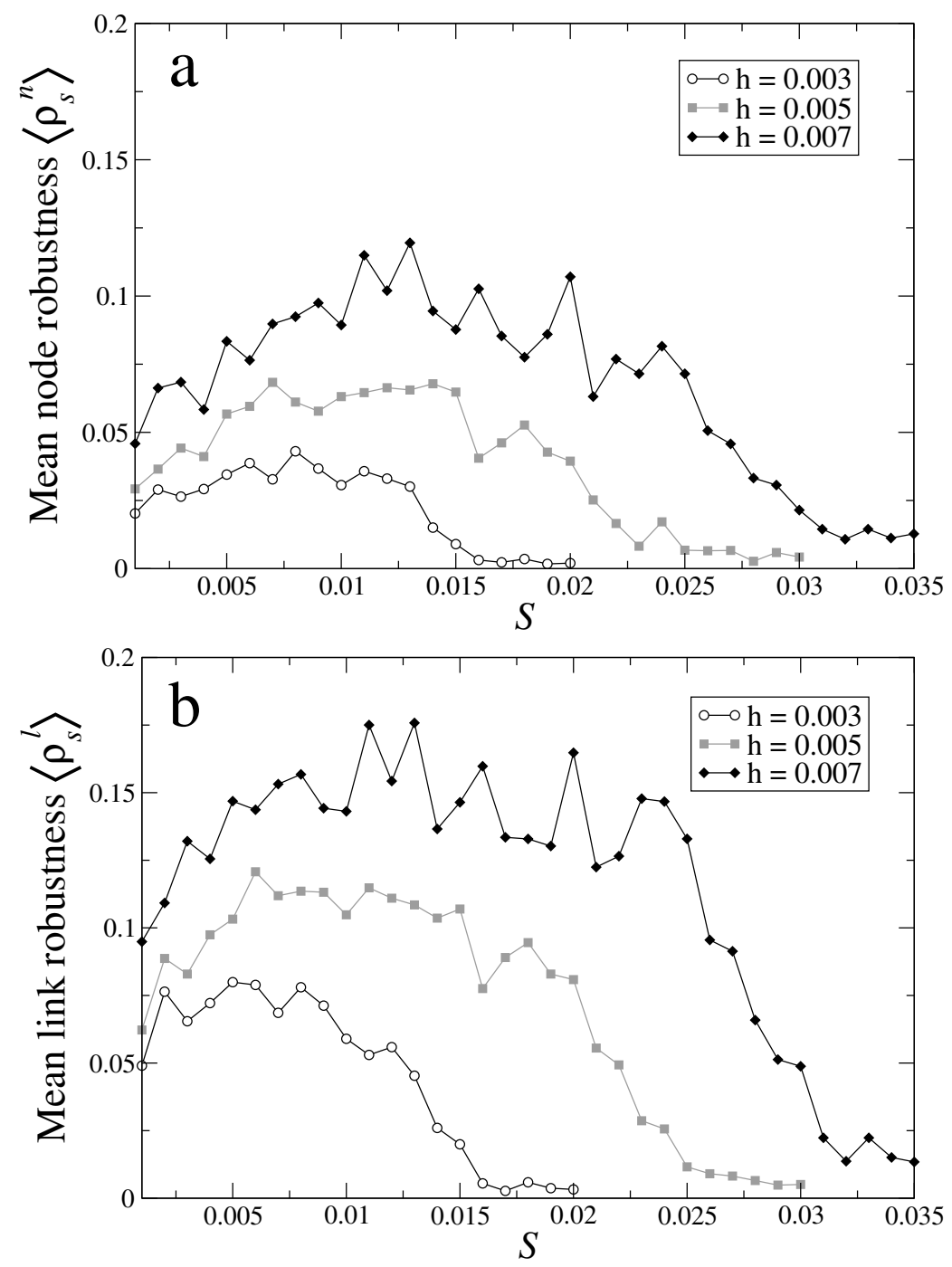

Figure 5.15: (a) Mean node $\left\langle\rho_{s}^{n}\right\rangle$ and (b) link $\left\langle\rho_{s}^{l}\right\rangle$ robustness of the networks optimized for noise robustness, as function of the noise intensity.

now, whether networks optimized for noise robustness are, perhaps, automatically robust against node removals and vice versa.

Figure 5.15 shows the mean node robustness $\left\langle\rho_{s}^{n}\right\rangle$ and the mean link robustness $\left\langle\rho_{s}^{l}\right\rangle$ 


\subsection{COMPARISON OF CROSS-ROBUSTNESS OF VARIOUS DESIGNED NETWORKS83}

of the networks optimized against noise. We can see that the networks optimized against noise are not robust against local damages (deletion of single nodes or links). Their robustness against such local damage is even less than for the networks optimized only by flow error $\left(\left\langle\rho_{f}^{n}\right\rangle \approx 0.5\right.$ and $\left\langle\rho_{f}^{l}\right\rangle \approx 0.7$, see Fig. 4.6 and 4.7$)$.

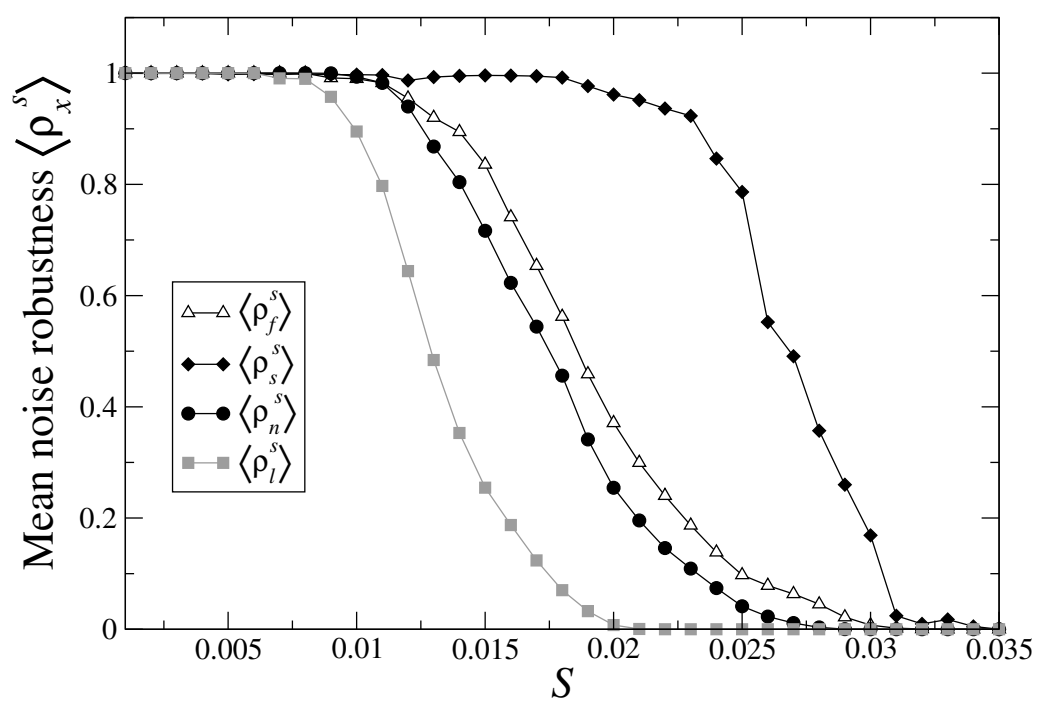

Figure 5.16: Mean noise robustenss $\left\langle\rho_{x}^{s}\right\rangle$ as function of the noise intensity $S$ for the networks optimized using different criteria of robustness. The threshold is $h=0.007$.

Figure 5.16 displays mean noise robustness of the networks optimized against noise $\left\langle\rho_{s}^{s}\right\rangle$, only by flow error $\left\langle\rho_{f}^{s}\right\rangle$, for node robustness $\left\langle\rho_{n}^{s}\right\rangle$ and for link robustness $\left\langle\rho_{l}^{s}\right\rangle$. In all cases, the noise robustness of the networks optimized against local damages is smaller than that of the networks optimized only by flow error and much smaller than the robustness of the networks optimized against noise. Note that the networks optimized for link robustness are particularly weak with respect to the noise. That can be seen as the consequence of the longer paths between the input and output nodes in such networks (Fig. 4.10).

The results of our study, based on a toy model of signal tranduction, suggest that robustness against distributed structural noise is not consistent with robustness against link removals. If a network is selected to yield robustness against link removals, it would usually show only low robustness against distributed noise. To solve the inconsistency problem and ensure robustness against noise, dynamical feedbacks may actually be employed in genetic networks of biological cells (see, e. g. [50]). 


\section{Chapter 6}

\section{Evolutionary construction of networks with required Laplacian spectra}

In this chapter, we concentrate our attention on the problem of reverse engineering of networks, considering, as motivation, first-order chemical reactions. Relaxation dynamics of such systems is determined by eigenvalues of some Laplacian matrices. Using an evolutionary optimization method, it is possible to find networks with the same eigenvalues and, therefore, the same dynamical properties as the prescribed targets. Many cospectral networks with the same spectra are however then found. We investigate their properties and look what additional information may by needed in order to identify a particular network.

\subsection{Laplacian spectra and relaxation phenomena in chemical networks}

Complex chemical processes in biological cells usually represent networks of crosscoupled chemical reactions. To control such processes, it is important to know the structure of a network. Reconstruction of the network structure from the set of experimental data, specifying the performance of a system under various conditions, is known as reverse engineering of chemical networks. This is a broad field of research where different approaches can be applied. In our study, we consider only networks of first-order chemical reactions $\left(X_{i} \rightarrow X_{j}\right.$ for $\left.i, j=1, \ldots, N\right)$. Such a network is characterized by a set of reaction rates $k_{j i}$, specifying the rate of conversion of a species $X_{i}$ into another species $X_{j}$. To simplify the problem, we assume that the rate constants of all reactions are equal (and choose this rate constant to be unity). Hence, the considered chemical networks are defined by directed graphs $G$ with adjacency matrices $\mathbb{A}$. A reaction $X_{i} \rightarrow X_{j}$ is present if $\mathbb{A}_{j i}=1$ and absent if $\mathbb{A}_{j i}=0$. Generally, this graph is asymmetric, so that $\mathbb{A}_{i j} \neq \mathbb{A}_{j i}$. 
Kinetic equations for concentrations $c_{i}$ of chemical species $X_{i}$ in the system are linear:

$$
\dot{c}_{i}=\sum_{j=1}^{N} \mathbb{A}_{i j} c_{j}-\sum_{k=1}^{N} \mathbb{A}_{k i} c_{i} .
$$

Their general solution has the form

$$
c_{i}(t)=\sum_{j=1}^{N} w_{j}^{(i)} e^{\lambda_{j} t}
$$

where coefficients $w_{j}^{(i)}$ are determined by initial conditions and the exponents $\lambda_{i}$ are given by eigenvalues of the Laplacian matrix $\mathbb{T}$ with the elements

$$
\mathbb{T}_{i j}=\mathbb{A}_{i j}-\delta_{i j} \sum_{k=1}^{N} \mathbb{A}_{k i} .
$$

The chemical system always possesses an equilibrium steady state, which corresponds to the zero eigenvalue $\lambda=0$ of matrix $\mathbb{T}$. This steady state is stable, so that $\operatorname{Re}\left(\lambda_{i}\right)<0$ for any other eigenvalue $i$.

By performing experiments and testing the response of a network to various perturbations, the set of exponents $\lambda_{i}$ can be determined from the relaxation data. In our study, we address a question whether the directed graph $G$ of a chemical network can be reconstructed from the relaxation data, i.e. whether the adjacency matrix $\mathbb{A}$ can be reconstructed when the spectrum $\lambda_{i}$ of the corresponding Laplacian matrix $\mathbb{T}$ is known.

Generally, the answer is no. There are cospectral graphs, which have the same spectra though their structure is different. However, if some restrictions are imposed and additional information about a graph is used, networks can be reconstructed with relatively high probability.

\subsection{Evolutionary construction method}

Reconstruction of a graph $G$ from a set of eigenvalues $\{\lambda\}_{0}$ can be done through an evolutionary process of selections and mutations, by using a variant of Metropolis algorithm that is similar to that which has been used in the previous chapters. We minimize the cost function $\epsilon$, representing the spectral distance between the set of eigenvalues $\{\lambda\}_{0}$, which we want to reconstruct, and the set of eigenvalues $\{\lambda\}_{G}$ of the graph $G$ which is evolving.

In the considered examples, the target set of eigenvalues $\{\lambda\}_{0}$ corresponds to a known graph $G_{0}$, to ensure that the solution of the construction problem is possible. This also 
allows us to compare the constructed (cospectral) graphs with the graph $G_{0}$ chosen for reconstruction.

\subsubsection{Spectral density and spectral distance}

To measure the distance between two sets of Laplacian eigenvalues, we first define the spectral density $\rho(G)$ corresponding to a Laplacian spectrum:

$$
\rho_{G}(z)=\sum_{i=1}^{N-1} \frac{\gamma}{\left|\lambda_{i}-z\right|^{2}+\gamma^{2}} .
$$

Here, $\lambda_{i}$ are Laplacian eigenvalues of a graph $G$ of size $N$. The parameter $\gamma$ gives the width of the Lorentz distributions. Because the zero eigenvalue is not included, there are only $N-1$ eigenvalues. Figure 6.1 gives an example of the spectral density of a graph of size $N=7$ with 10 connections.

a

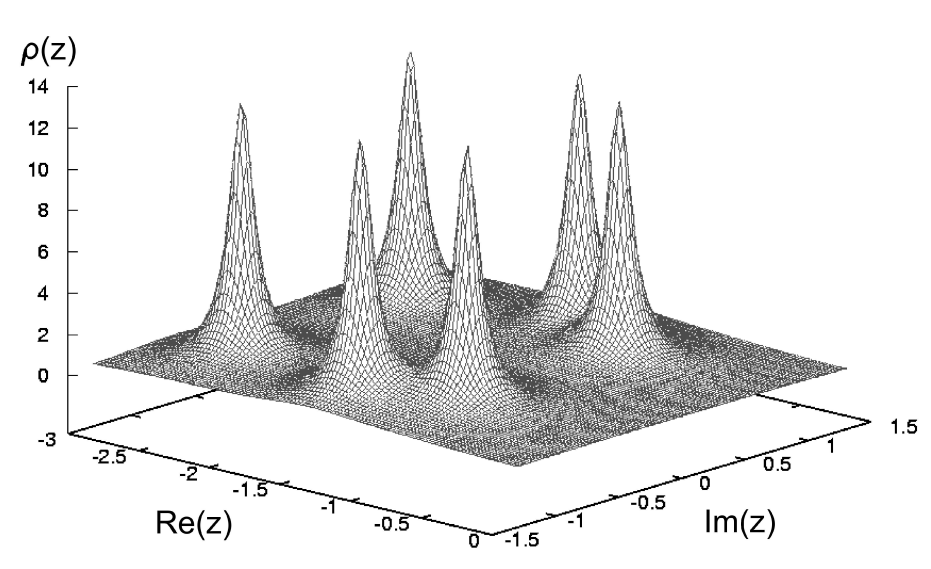

b

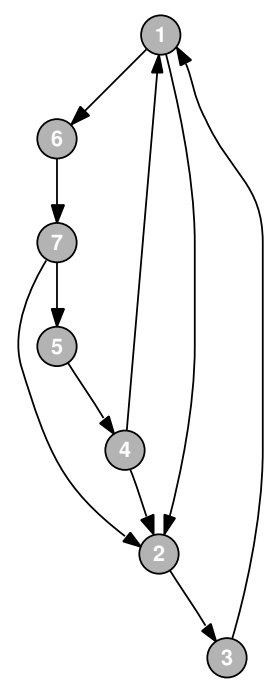

Figure 6.1: Spectral density (a) of the graph (b). The eigenvalues are $0,-0.981 \pm$ $0.603 i,-1.5 \pm 0.866 i$ and $-2.519 \pm 0.667 i$. Here, we have $\gamma=0.08$.

The spectral distance $\epsilon$ between two graphs of the same size $N$ is defined as

$$
\epsilon_{A B}=\frac{1}{K} \sqrt{\int^{z}\left[\rho_{A}(z)-\rho_{B}(z)\right]^{2} d z},
$$

where $\rho_{A}(z)$ and $\rho_{B}(z)$ are spectral densities of the graphs $A$ and $B$ and $K$ is the normalization constant. 
The maximum spectral distance is obtained when each set of eigenvalues has only one element (which is $N-1$ times degenerate) and these two elements are far away. Such a situation is found, for example, when we consider the distance between the disconnected and the full-connected graphs of the same size. In this case, we have

$$
\epsilon_{\max }=\frac{1}{K} \sqrt{2 \pi(N-1)^{2}}
$$

We require that $\epsilon_{\max }=1$ and fix therefore the normalization constant to be

$$
K=\sqrt{2 \pi(N-1)^{2}}
$$

Thus, the distance may vary only within the interval between zero and unity.

\subsubsection{Evolutionary optimization algorithm}

A variant of the Metropolis algorithm is used to make reconstructions. Given the target set of eigenvalues $\left\{\lambda_{i}\right\}_{0}$ of a graph $G_{0}$, we let the graph $G$ to evolve reducing the spectral distance between the two graphs. In each iteration, the following steps are performed:

1. An evolutionary mutation is applied to the graph $G$ with spectral distance $\epsilon$, yielding a new graph $G^{\prime}$ with the spectral distance $\epsilon^{\prime}$

2. The difference $\Delta \epsilon=\epsilon^{\prime}-\epsilon$ is computed.

3. If $\Delta \epsilon<0$, we accept the mutation, replacing $G$ by $G^{\prime}$. If $\Delta \epsilon>0$, the mutation is accepted with the probability $\exp (-\Delta \epsilon / \sigma \epsilon)$.

4. Return to step 1.

We run the evolution until a graph with the required Laplacian spectrum $(\epsilon=0)$ is obtained or for a fixed number of iterations. The temperature parameter $\sigma$ affects the performance of the optimization. Therefore, we have to find its optimum value.

As the initial condition in each evolution, we use a random Erdös-Rényi graph, with a connectivity $p$ which is randomly chosen in each new simulation. In this optimization algorithm, the temperature $T=\sigma \epsilon$ decreases during the evolution, so that this optimization method corresponds to simulated annealing [49].

\subsubsection{Evolutionary mutations}

Several evolutionary mutation schemes have been tested by us. Here, we show two mutation schemes with the best performance. The first mutation scheme, involving node 
mutations, consists in choosing randomly a node $i$ in the graph $G$ and deleting all of its incoming and outgoing connections. After that, the sets of new $d_{\text {in }}$ incoming and $d_{\text {out }}$ outgoing connections for this node are generated. The numbers $d_{\text {in }}$ and $d_{\text {out }}$ and the node i are randomly chosen in each next mutation

In the second mutation scheme, involving cycle mutations, directed cycles in the graph $G$ are changed. The mutation consists of generating a random cycle $g_{M}$ of length $M$ $(2 \leq M \leq N)$, which goes through the nodes $n_{1}, \ldots, n_{M}$. The length and the particular nodes, forming the cycle, are randomly chosen. The cycle can be added to the graph $G$ or deleted from it, with the same probability. Adding the cycle means that we add to the graph $G$ all connections which are present in the cycle $g_{M}$. Deleting the cycle means that we remove all connections which are present both in the graph $G$ and in the cycle $g_{M}$. Figure 6.2 gives an example of this mutation scheme.

$\mathbf{a}$

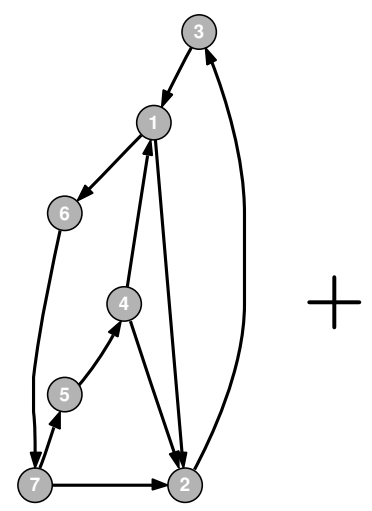

$G$

b

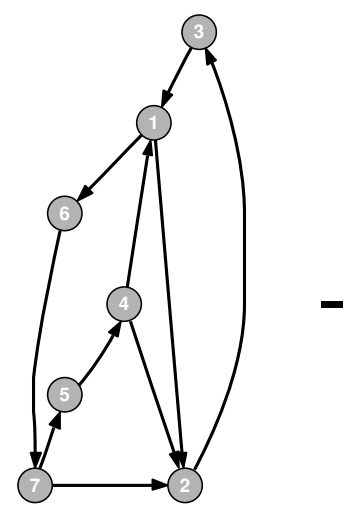

G

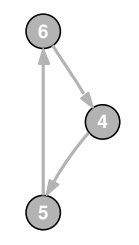

$g_{M}$

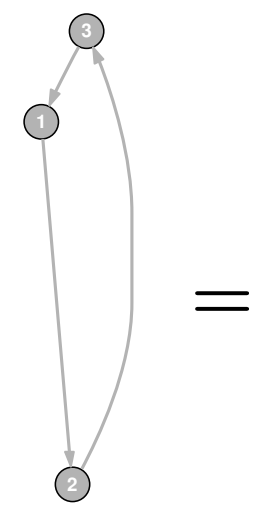

$g_{M}$

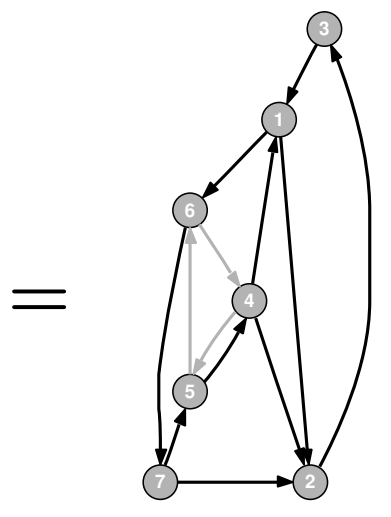

$G^{\prime}$

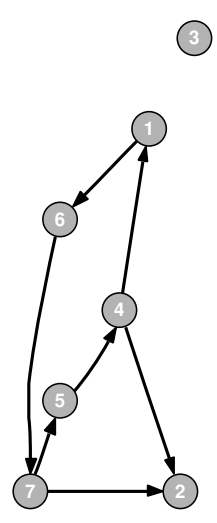

$G^{\prime}$

Figure 6.2: Example of a cycle mutation. (a) The cycle $g_{M}$ is added to the graph $G$ and (b) the cycle $g_{M}$ is deleted from the graph $G$, yielding a new graph $G^{\prime}$.

We will see that the cycle mutation scheme has a better optimization performance than the node mutations. 


\subsubsection{Example of spectral reconstruction}

We present an example of reconstruction from the Laplacian spectra, using the spectrum of a graph $G_{0}$ of size $N=10$ with 15 connections. Figure 6.3 a shows the evolution of the spectral distance $\epsilon$ during the reconstruction. As the initial condition, a random Erdös-Rényi graph of size $N=10$ with connectivity $p=0.3$ is chosen. The reconstruction has been performed, until a graph (Fig. 6.3c) with the same Laplacian spectra $(\epsilon=0)$ as the graph $G_{0}$ was found. In this example, we have used the cycle mutation scheme

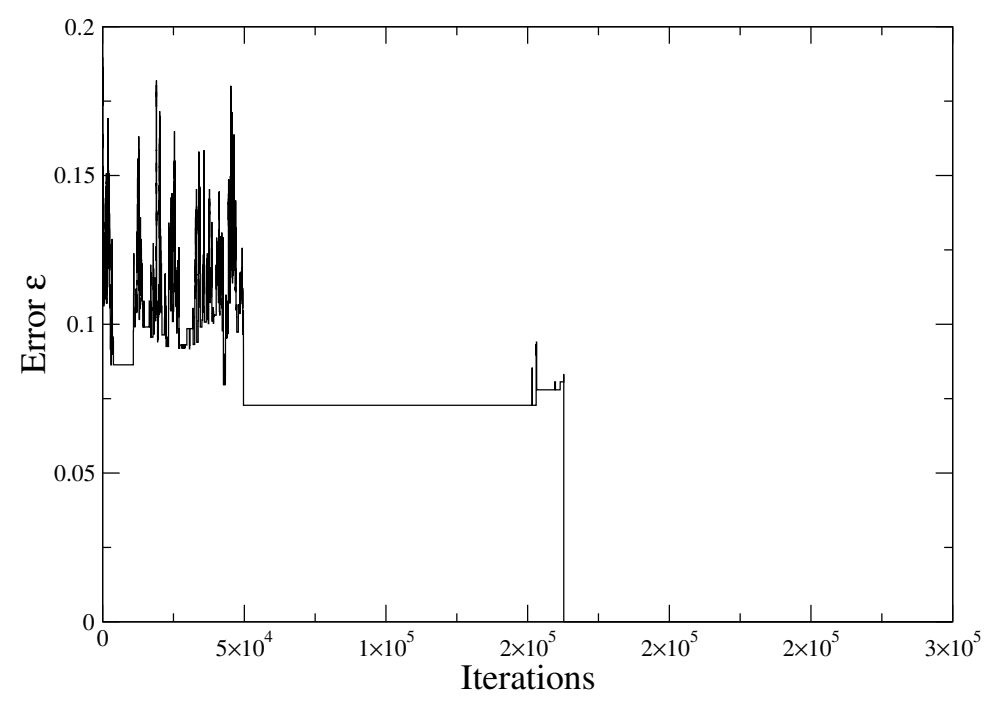

a

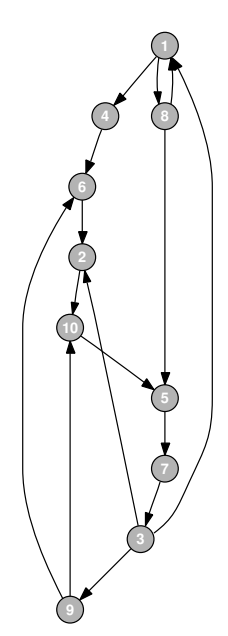

b

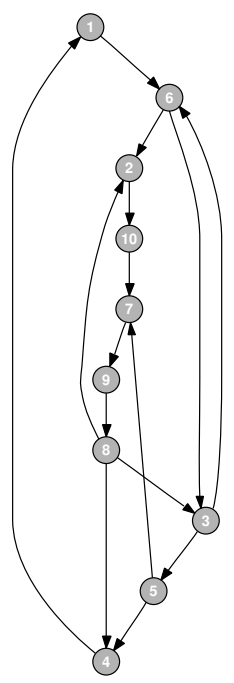

C

Figure 6.3: Example of an evolution. (a) The spectral distance $\epsilon$ as a function of the number of iterations. (b) The target graph, used to calculate the set of Laplacian eigenvalues, and (c), the final obtained graph. The temperature parameter $\sigma=4.73 \times 10^{-2}$.

during the evolutionary optimization. The final obtained graph (Fig. 6.3c) and the target one (Fig. 6.3b) are cospectrals, they have the same Laplacian spectra, but different topologies.

Optimal values of the temperature parameter $\sigma$ for two different mutations schemes have been found in this example. We have performed evolutions with different values of this parameter and with random initial graphs. Given a value of $\sigma$ and the mutation scheme, we have constructed a graph with the Laplacian spectrum of the graph $G_{0}$, using ensembles of 2500 initial random graphs each and running the evolutions for $5 \times 10^{5}$ iterations.

Figure 6.4a shows the mean spectral distances $\langle\epsilon\rangle$ of the graphs in the ensembles after the evolutions as a function of the temperature parameter $\sigma$ for both schemes of mutations. For node mutations (gray squares), the performance corresponding to the smallest mean error is found at $\log (\sigma)=-1.75(\sigma \approx 0.017)$. For cycle mutations (black 
circles), the best temperature parameter is $\log (\sigma) \approx-1.5(\sigma \approx 0.037)$.
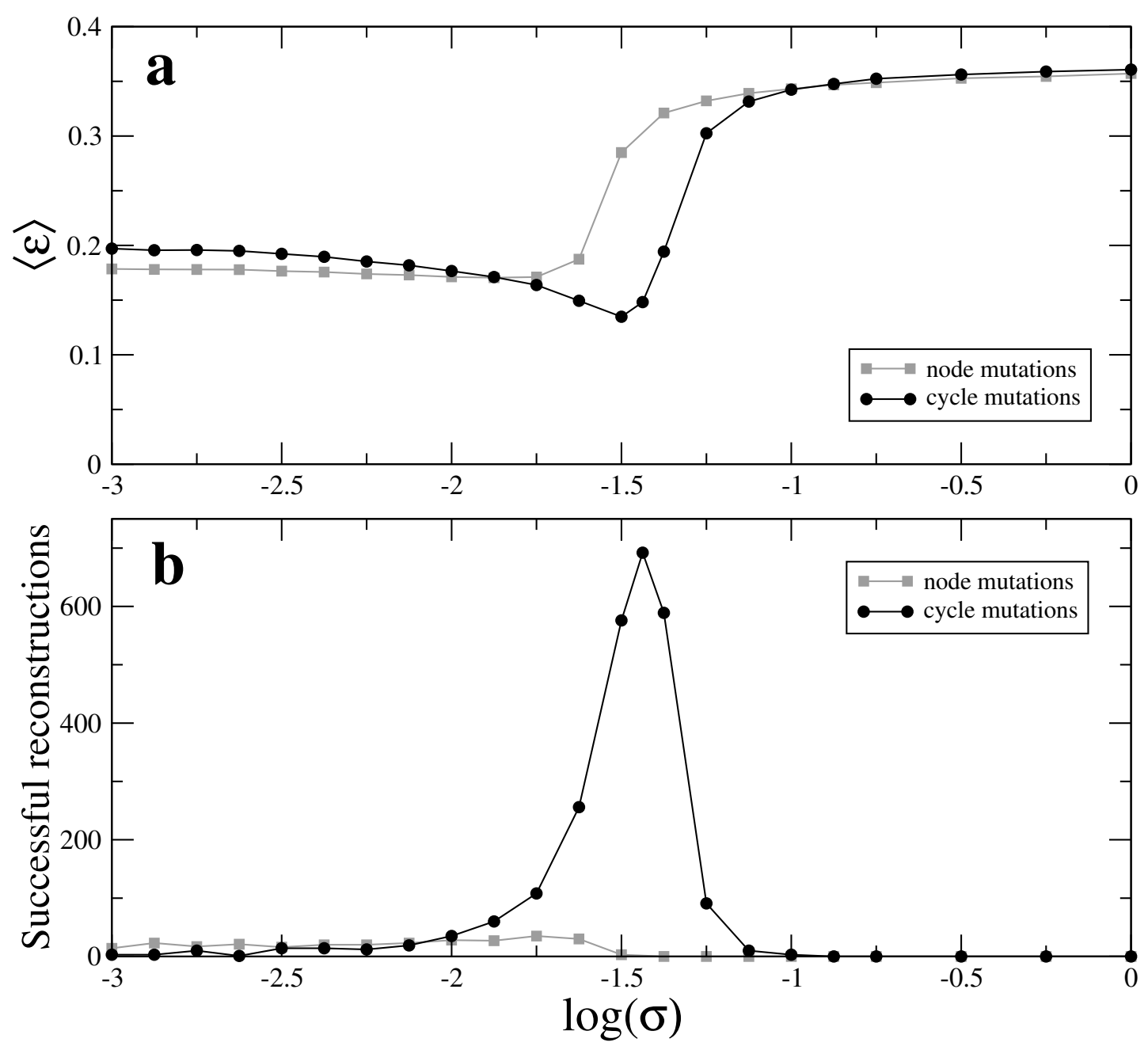

Figure 6.4: (a) Mean spectral distance $\langle\epsilon\rangle$ as a function of $\sigma$ for two mutation schemes. Networks constructed using node mutations (squares) and cycle mutations (circles). (b) The number of successful constructions as a function of $\sigma$. Each point corresponds to the constructions from the Laplacian spectrum of the same graph $G_{0}$, using 2500 initial random networks and $5 \times 10^{5}$ iterations.

Figure $6.4 \mathrm{~b}$ presents the number of successful constructions, i. e. the number of graphs with $\epsilon=0$ in each ensemble, as a function of the temperature parameter $\sigma$. The best performance corresponds to $\log (\sigma)=-1.375$ and for using the cycle mutation scheme. In the case of the node mutation scheme, the performance has been low in the whole range of temperature parameters, even when the reconstructions have been possible $(\log (\sigma)<-1.5)$. 


\subsection{Construction of families of cospectral graphs}

The final graph in Fig. 6.3 had the same Laplacian spectrum, as the target graph, but its architecture was different. We have found that this situation is typical. Usually, an evolutionary reconstruction of a graph from its spectrum leads to a different graph, which is however cospectral with the target. Because there are so many cospectral graphs, the Laplacian spectrum does not well define the network architecture and recovering the structure of a network from the spectral data is not practically possible.

However, the evolutionary optimization method can still have interesting applications. It can be used to construct families of the graphs which are cospectral to a given graph. Indeed, no rational methods for construction of cospectral graphs are known. Moreover, one cannot also just find the spectra of all possible graphs and thus determine which of them are cospectral, since the total number of graphs of even relatively small size is extremely large. When families of cospectral graphs are found, statistical investigations of their properties can be performed.

Cospectral graphs must be distinguished from isomorphic graphs, which have the same architecture and differ only by a change in the numeration of their nodes (i. e., by a permutation). Determining if two graphs are isomorphic can be done by some algorithms in a polynomial time. Below, we compare properties of cospectral graphs which are invariant under permutations of the adjacency matrices.

To characterize graphs, eigenvalues and singular values of their matrices can be used. For a square matrix $\mathbb{B}$, the square roots of the eigenvalues of $\mathbb{B}^{H} \mathbb{B}$, where $\mathbb{B}^{H}$ is the conjugate transpose, are called singular values. If two graphs have matrices $\mathbb{A}$ and $\mathbb{T}$ with the same eigenvalues and singular values respectively, we say that they are isomorphic. Whereas, if any of such sets are different, they are cospectrals.

As an example, Fig. 6.5 shows 41 graphs, which are cospectral to the graph given in Fig. 6.3b. They were obtained by running independent evolutionary constructions and discarding isomorphic graphs.

\subsubsection{Structural statistical properties of cospectral graphs}

Using as an example the family of 41 cospectral graphs (Fig. 6.5), structural statistical properties of cospectral graphs can be discussed. Figure 6.6 displays histograms of several statistical structural properties of these graphs.

The distribution of clustering coefficients for such graphs is shown in Fig. 6.6a. There are 11 different values of this coefficient, but for many cospectral graphs (20) the clustering coefficient is zero.

The properties, related to paths in these networks, are the diameter $(L)$ and the average path length $(A P L)$. The diameter, which represents the longer distance between any two nodes in the graph, is shown in Fig. 6.6b. It takes four values, but 30 graphs have 

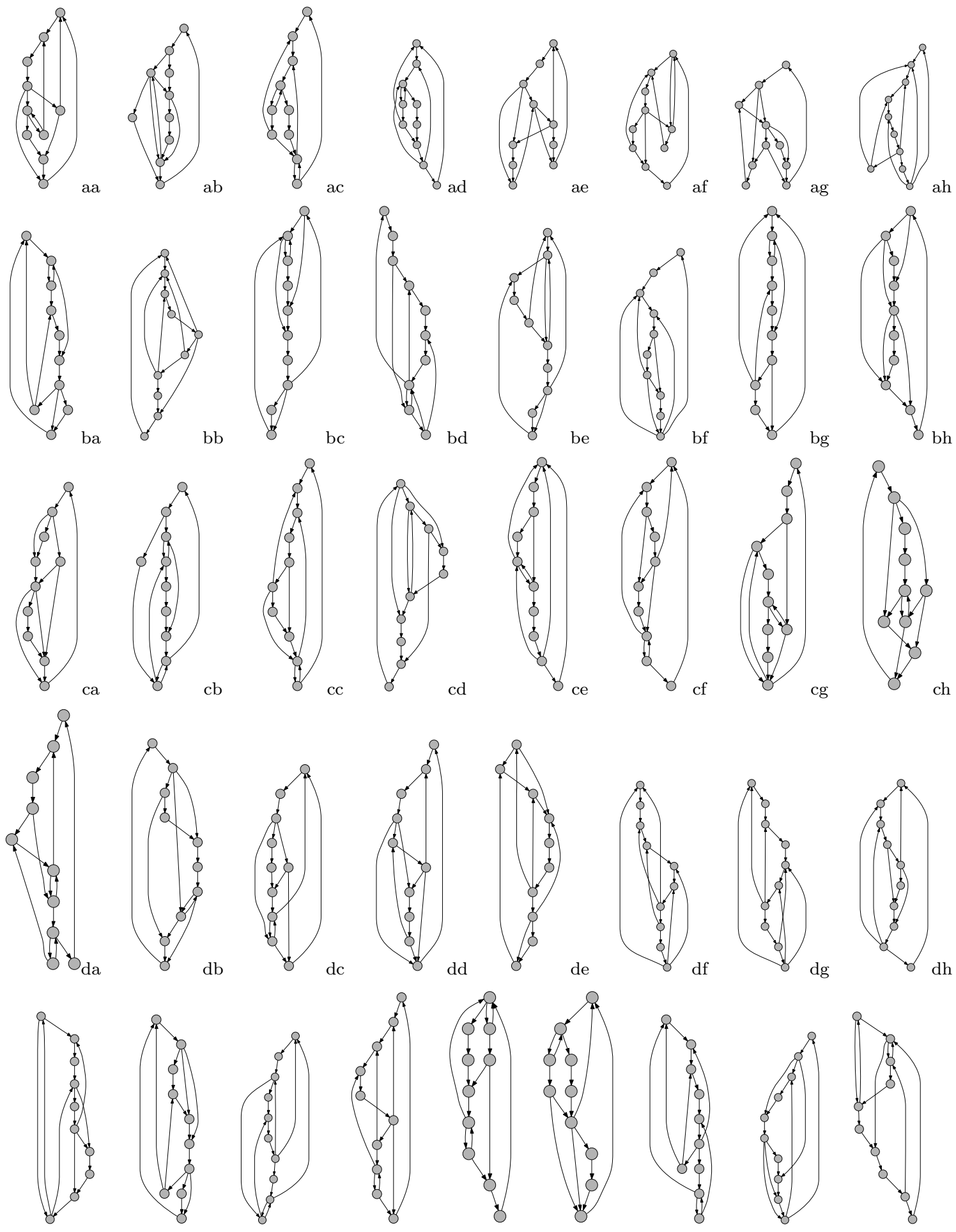

ea

eb

ec

ed

ee

ef eg

Figure 6.5: A family of 41 cospectral graphs. All these graphs have the same Laplacian spectra as the graph shown in Fig. 6.3b. 

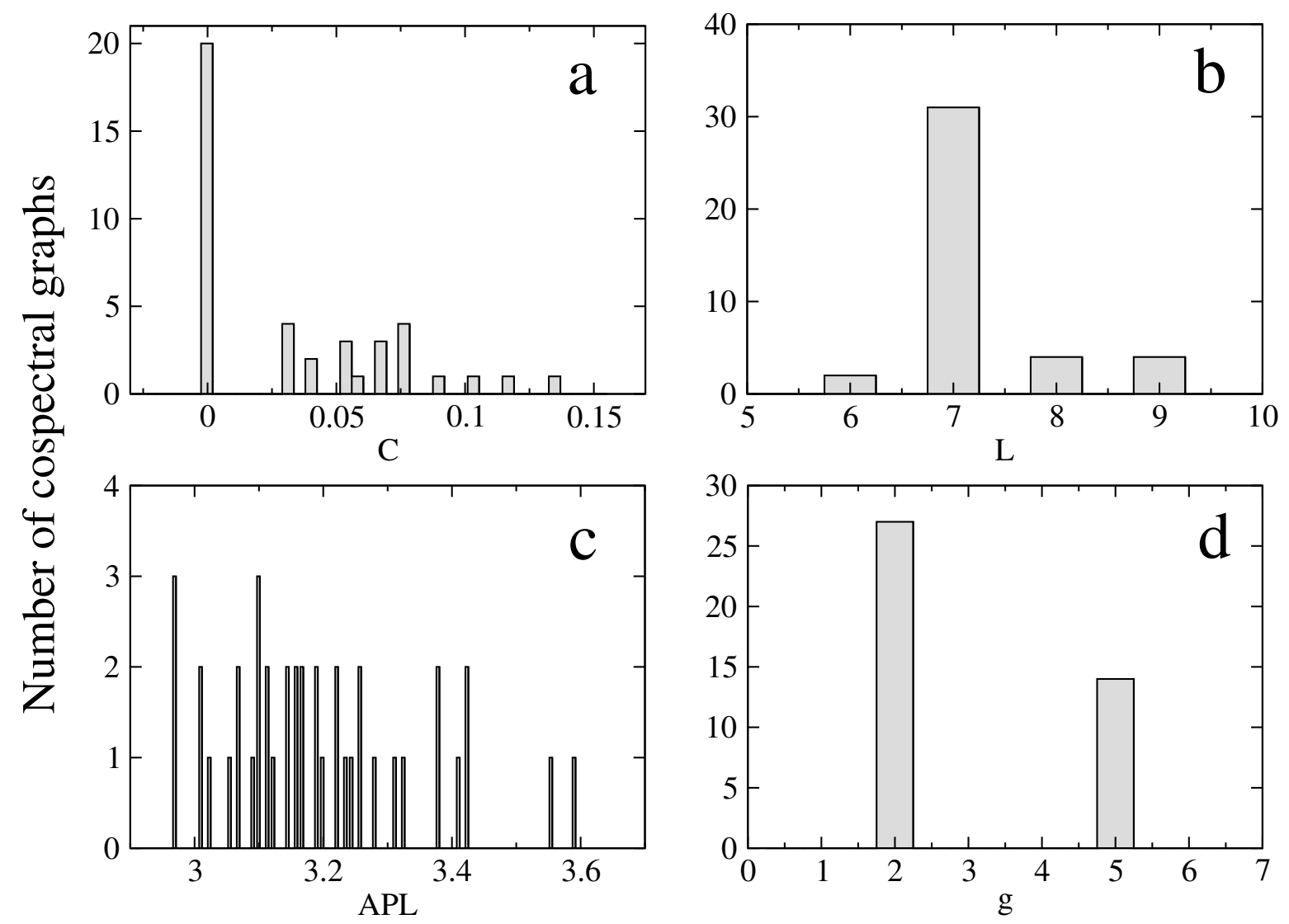

Figure 6.6: Histograms of structural properties of the constructed 41 cospectral graphs: (a) clustering coefficient, (b) diameter, (c) average path length and (d) girth.

the same diameter $L=7$. The average path length, i. e. the average distance between all pairs of nodes in the graph, is shown in Fig. 6.6c. Here, a large diversity of values is seen.

Finally, Fig. 6.6d shows the distribution of girths. The girth $g$ is defined as the length of the smallest directed cycle present in a graph. Only two possible girth values are found in these cospectral graphs.

\subsubsection{Comparison with non-directed graphs}

A similar study of evolutionary reconstruction has been previously performed for nondirected graphs [51]. In this case, the Laplacian spectrum usually determines the architecture of a graph, because the number of cospectral graphs is very small. For example, for $N \leq 6$ there is only one pair of connected cospectral graphs. For regular graphs (with equal degrees of all nodes), one pair of size 12, two pairs of size 16, a cospectral quadruple of size 28, seven cospectral pairs of size 25, and a set of 91 cospectral graphs of size 35 are known [52]. For non-directed graphs, evolutionary optimization methods can be 
therefore used to reconstruct a graph from its spectral data or to obtain graphs with the architectures similar to that of a graph used for the reconstruction.

A large number of cospectral graphs does not allow to use the same approach in the case of a directed graph. In the next section, we analyze, why the frequency of cospectral graphs is much higher for the networks with directed connections than for the non-directed graphs.

\subsection{Origin of cospectral directed graphs}

Starting from a given directed graph and adding a single node, many cospectral graphs can easily be constructed. As an example, we can take a source node (without any incoming connections) that has $g$ outgoing connections. Adding such a node generates a new Laplacian eigenvalue $\lambda=-g$. This eigenvalue remains the same, independent of what $g$ nodes in the graph become connected to the new added node. Therefore, all graphs obtained by choosing different $g$ nodes connected to the new source node will have the same Laplacian spectra.

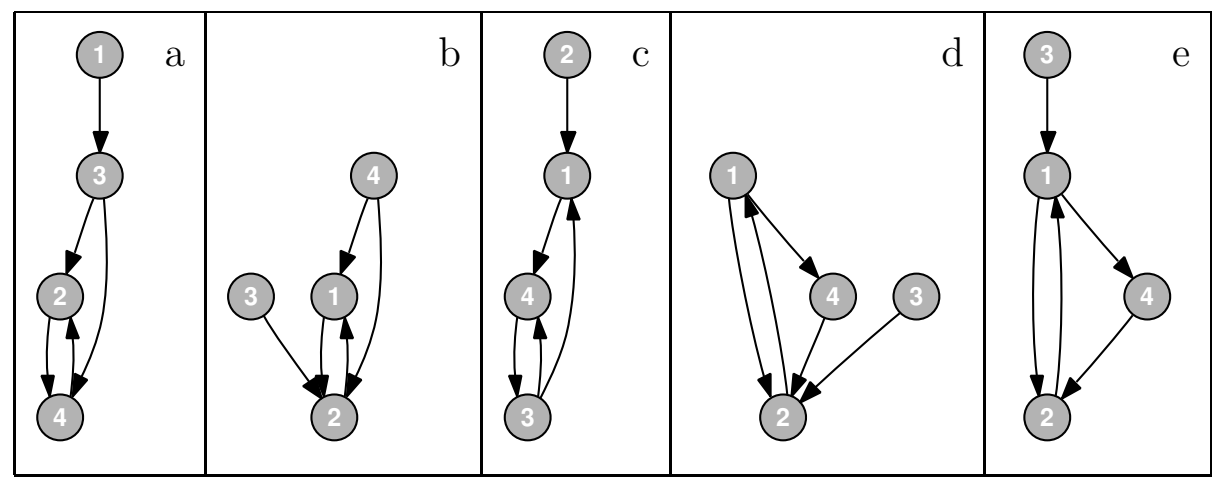

Figure 6.7: Cospectral graphs with Laplacian eigenvalues $\{-2,-2,-1,0\}$.

As an illustration, five cospectral graphs in Fig. 6.7 are constructed by adding a source node with a single outgoing connection $g=1$ to different nodes of the same graph. In Fig. 6.8, further examples are given. Here, the graphs are constructed by adding two sources nodes (Fig. 6.8a), by adding a source node with two outgoing connections (Fig. 6.8b-e), or by using a strongly connected diagraph (Fig. 6.8f). Note that all graphs shown in Fig. 6.7 and Fig. 6.8 have the same set $\{-2,-2,-1,0\}$ of Laplacian eigenvalues and are therefore cospectral.

Generally, cospectral graphs can be obtained by adding any source subgraph to a graph. The source subgraph $S$ should have only outgoing connections to the preexisting graph $G^{\prime}$. The adjacency matrix $\mathbb{A}_{G}$ of the new graph $G$ will have the block structure

$$
\mathbb{A}_{G}=\left(\begin{array}{cc}
\mathbb{A}_{S} & 0 \\
\mathbb{C} & \mathbb{A}_{G^{\prime}}
\end{array}\right)
$$




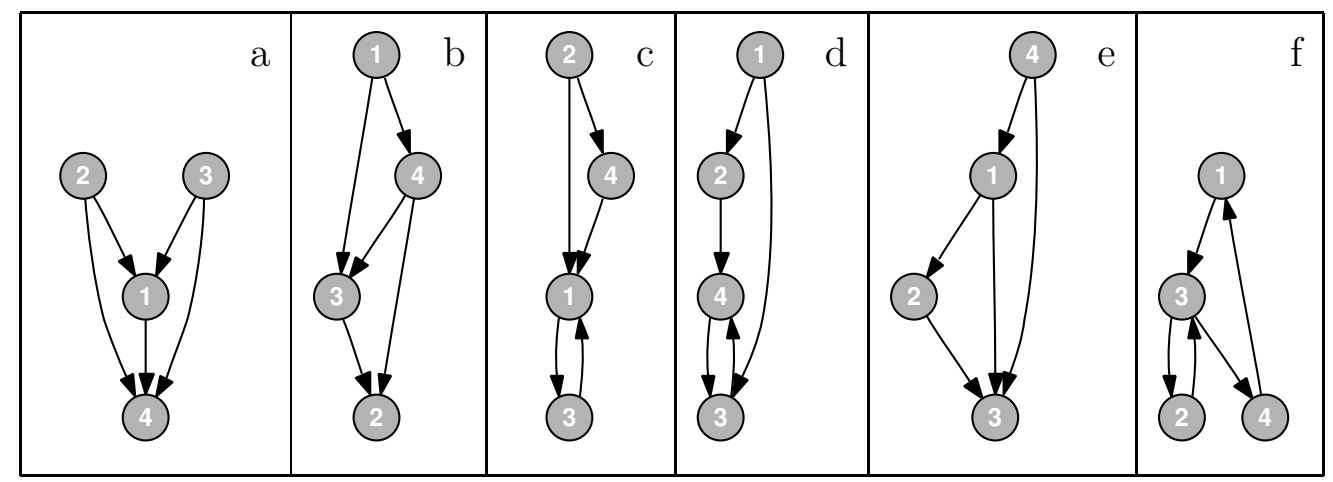

Figure 6.8: Further examples of cospectral graphs with the Laplacian eigenvalues $\{-2,-2,-1,0\}$.

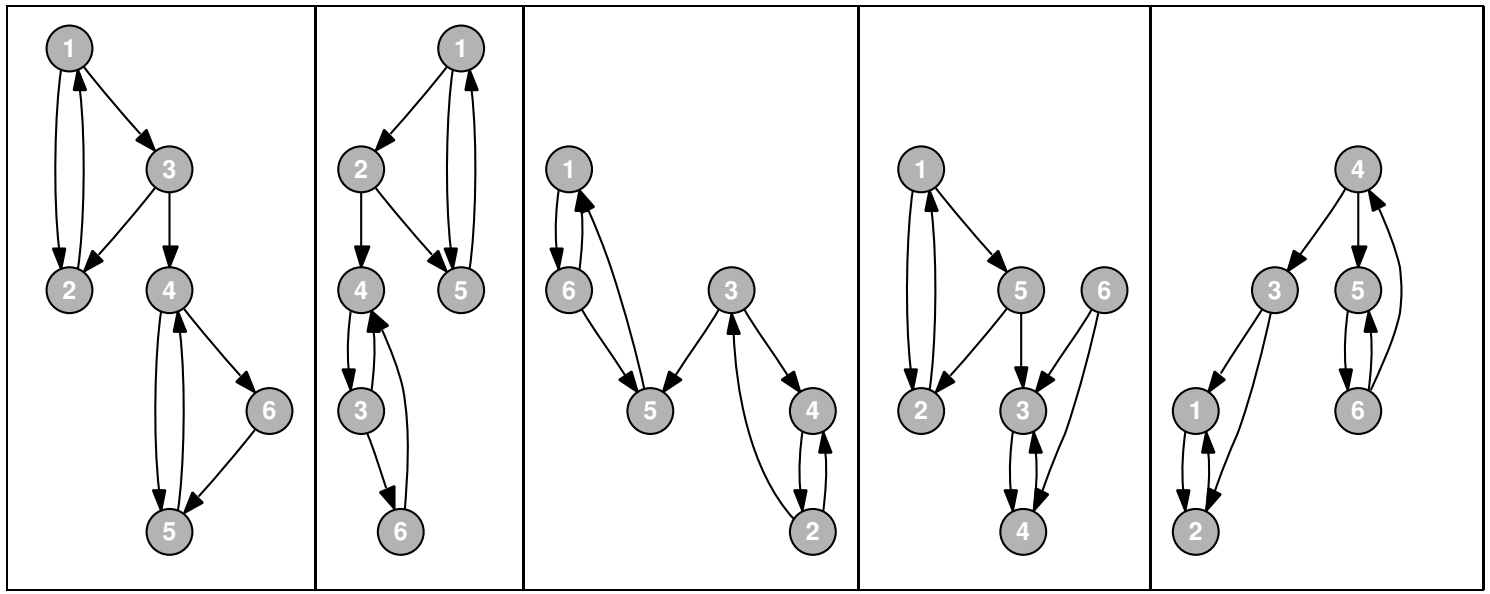

Figure 6.9: Cospectral graphs with Laplacian eigenvalues $\{-2.42 \pm$ $0.61 \imath,-2,-2,-0.16,0\}$.

Clearly, the determinant of such a matrix is independent of the values of the elements in the connection block $\mathbb{C}$. Thus, all such graphs $G$ will be cospectral. Figure 6.9 shows 5 cospectral graphs obtained by taking two subgraphs with 3 nodes and using one of them as the source subgraph. Although the elements of the block $\mathbb{C}$ do not appear in the determinant of the matrix $\mathbb{A}_{G}$, we can change their positions only inside their same columns, otherwise the outgoing degrees of the nodes of the source $S$ (diagonal elements of block $\mathbb{A}_{S}$ ) would become changed and then, the Laplacian eigenvalues could also be changed.

The number of possible cospectral graphs can be reduced if we consider only strongly connected diagraphs. In such networks, any node can be reached from any other node by traversing connections in the directions into which they point. In such graphs, sources or sinks are not allowed. Therefore, generation of cospectral graphs by adding source subgraphs is not then possible. However, there is a large number of cospectral graphs even in this case. Note that all 41 cospectral graphs in Fig. 6.5 actually represent 
strongly connected directed graphs. 


\section{Conclusions}

In this work, we have shown how networks with predefined functions and robust against different kinds of local damages and noise can be constructed. We have found that the structures of these networks strongly depend on the criteria of robustness considered during their constructions. The principal results of our investigations have already been published in two research articles [53, 54] and in a book chapter [55].

Chapters 2, 3, 4 and 5 were devoted to the construction of functional and robust networks. The flow distribution model, formulated in Chapter 2, is convenient for the statistical analysis, because its rules are simple and, therefore, the simulations are not computationally demanding. Moreover, its operation depends only on the network architecture, which has allowed us to focus on the structural properties of the designed networks.

In Chapter 3, a variant of the Metropolis algorithm was applied to minimize flow errors of the networks, indicating the difference between the actual output and the target output patterns (i. e., the prescribed function). The performance of this optimization algorithm depends on the scheme of evolutionary mutations used. We have found that, using a scheme of mutation based on the addition or substraction of a path, the convergence is faster than for the other scheme of mutations based on the addition or substraction of a single link. Moreover, some structural differences in the networks obtaining by using these two schemes of mutations have been found. Therefore, the choise of evolutionary mutations is important. For subsequent investigations, the path mutation scheme has been chosen, as yielding the faster convergence.

Chapter 4 was dedicated to the study of the effects of local damages in flow distribution networks. The evolutionary optimization algorithm, presented in Chapter 3, was modified in order to not only decrease the flow error, but also to increase robustness of the evolving networks. We have considered two kinds of local damages: removal of a randomly chosen middle node and removal of a randomly chosen link. It was found that the networks optimized with respect to these two different criteria of robustness have different structural properties and motif distributions. The networks robust against node deletions possess less connections than the networks robust against the removal of links. The most remarkable difference was seen, however, in motif distributions of these networks. The networks robust against link deletions have the motif distribution belonging to the second superfamily. This superfamily including signal transduction and genetic developmental 
networks in biological macroorganisms, as well as the neural network of the primitive animal $C$. elegans. On the other hand, networks robust against node deletions have motif distributions which are different from real biological networks and similar to those of the networks of languages.

In Chapter 5, topological noise was introduced and flow distribution networks robust against such noise were constructed. The networks robust against noise exhibit structural properties, which are similar to those of the networks robust against the removal of nodes. Motif distributions of these networks are also similar to those of the networks robust against the removal of middle nodes. However, we have found that the networks robust against noise are not usually at the same time robust against the removal of nodes. These indicates that more fine structural differences between these two kinds of networks should be important.

Differences in the structures of various robust networks are directly imposed by the criteria of robustness used during the optimization process. In all cases, we have considered the same kinds of input-output relationships and, therefore, all designed networks have the same functions. Moreover, the same scheme of evolutionary mutation, have been employed in their constructions.

The observed similarity between motif distributions of the networks robust against the deletion of links and the biological networks of the second superfamily suggests that the architecture of these real systems can be controlled by robustness against failures in the interactions between the nodes, such as the deletion of a synapse or of a protein-protein interaction. Real biological systems should also be robust against noise. However, we have found that flow distribution networks robust against noise have different motif distributions. Calculating cross-robustness, we have found that flow distribution networks robust against noise are not robust against removal of links, and vice versa. The architectures of real biological networks could have evolved to be robust against links removals. Robustness against noise can further be achieved through the dynamics, by introducing negative feedback loops. In our model, such dynamical mechanisms of noise suppression are not possible because the flow distribution networks operate statically.

Finally, in Chapter 6 we have used a similar evolutionary optimization method in a reverse engineering problem of reconstruction of networks from their Laplacian spectra. In this case, differences in the performances of the two considered schemes of evolutionary mutations have been important. It has been found that the scheme of mutations based on the addition or substractions of randomly chosen cycles works much better than the other scheme. We have shown that the construction of networks with predefined Laplacian spectra is possible. However, their structure (i. e., the adjacency matrix) is not uniquely determined by such spectra and, therefore, many diferent cospectral graphs are obtained as the solution of the reconstruction problem. The number of cospectral directed graphs is large. This is explained by the special role of some structures, such as source subgraphs, which can easily generate different graphs with the same spectrum. The evolutionry optimization algorithm can be used to obtain families of cospectral graphs and investigate 
their statistical properties.

In the future, it would be interesting to consider extensions of the investigated flow distribution model where dynamical responses of a network are possible. This would allow to consider, for example,effects of dynamical noise and to study temporal output patterns of processed signals. 


\section{Bibliography}

[1] R. Albert and A. L. Barabási, "Statistical mechanics of complex networks", Reviews of Modern Physics $\mathbf{7 4}$ (2002).

[2] M. E. J. Newman, "The structure and function of complex networks", SIAM Review 45, 167 (2003).

[3] S. N. Dorogovtsev and J. F. F. Mendes, "Evolution of networks", Advances in Physics 51, 1079 (2002).

[4] D. Watts and S. Strogatz, "Collective dynamics of 'small-world' networks", Nature (London) 393, 440 (1998).

[5] P. Erdös and A. Rényi, "On random graphs", Publ. Math. 6 (Debrecen) 290 (1959).

[6] A. L. Barabási and R. Albert, "Emergence of scaling in random networks", Science 286, 509 (1999).

[7] Y. Kuramoto, Chemical Oscillations, Waves, and Turbulence (Springer, Berlin 1984).

[8] H. Kori and A. S. Mikhailov, "Entrainment of randomly coupled oscillator networks by a pacemaker", Phys. Rev. Lett. 93, 254101 (2004).

[9] Jörg Stelling, Uwe Sauer, Zoltan Szallasi, Francis J. Doyle, III, and John Doyle, "Robustness of cellular functions", Cell 118, 675-685 (2004).

[10] D. Deutscher, I. Meilijson, M. Kupiec and E. Ruppin, "Multiple knockout analysis of genetic robustness in the yeast metabolic network", Nature Genetics 38, 9 (2006).

[11] R. Albert, H. Jeong and A. L. Barabási, "Error and attack tolerance of complex networks", Nature 406, 378 (2000).

[12] D. S. Callaway, M. E. J. Newman, S. H. Strogatz and D. J. Watts, "Network robustness and fragility: percolation on random graphs", Phys. Rev. Lett. 85, 5468 (2000).

[13] T. Tanizawa, G. Paul, R. Cohen, S. Havlin and H. E. Stanley, "Optimization of network robustness to waves of targeted and random attacks", Phys. Rev. E 71, $047101(2005)$. 
[14] D. Armbruster, K. Kaneko, A. S. Mikhailov (eds.), Networks of Interacting Machines: Production Organization in Industrial Systems and Biological Cells, (World Scientific, Singapore 2005).

[15] J. G. White, E. Southgate, J. N. Thomson and S. Brenner, "The structure of the nervous system of the nematode Caenorhabditis elegans", Phil. Trans. R. Soc. London, B 314, 1 (1986)

[16] T. Ferrée and S. Lockery, "Computational rules for chemotaxis in the nematode $C$. elegans", J. Comp. Neurosci. 6, 263 (1999)

[17] R. Heinrich, B. G. Neel and T. A. Rapoport, "Mathematical models of protein kinase signal transduction", Molecular Cell 9, 957 (2002).

[18] S. J. Vayttaden, S. M. Ajay and U. S. Bhalla, "A spectrum of models of signaling pathways", ChemBioChem 5, 1365 (2004).

[19] H. M. Sauro and B. N. Kholodenko, "Quantitative analysis of signaling networks", Prog Biophys Mol Biol 86, 5 (2004).

[20] F. Schacherer, C. Choi, U. Gotze, M. Krull, S. Pistor and E. Wingender, "The TRANSPATH signal transduction database: a knowledge base on signal transduction networks", Bioinformatics 17, 1053 (2001).

[21] B. Binder and R. Heinrich, "Interrelation between dynamical properties and structural characteristics of signal transduction", Genome Informatics 15, 13 (2004).

[22] B. Binder and R. Heinrich, "Structural and dynamical analyses of the kinase network derive from the Transpath database", Genome Informatics 16, 164 (2005).

[23] T. S. Gardner and J. J. Faith, "Reverse-engineering transcription control networks", Physics of Life Reviews 2, 65 (2005).

[24] E. Lalli and P. Sassone-Corsi, "Signal transduction and gene regulation: The nuclear response to cAMP", The Journal of Biological Chemistry 269, 17359 (1994).

[25] C. H. Yeang, T. Ideker and T. Jaakkola, "Physical network models", J Comput Biol, 11, $243(2004)$.

[26] T. Chen, H. L. He, G. M. Church, "Modeling gene expression with differential equations", Pacific Symp. Biocomput. 4, 29 (1999).

[27] S. A. Kauffman, Origins of Order: Self-Organization and Selection in Evolution (Oxford: Oxford Univ. Press, 1989).

[28] T. Akutsu, S. Miyano and S. Kuhara, "Identification of genetic networks from a small number of gene expression patterns under the Boolean network model", Pacific Symp. Biocomput. 4, 17 (1999). 
[29] E. Segal, B. Taskar, A. Gasch, N. Friedman and D. Koller, "Rich probabilistic models for gene expression", Bioinformatics 1, 243 (2000).

[30] A. S. Mikhailov and A. Yu. Loskutov, Foundations of Synergetics II: Chaos and Noise (Springer Series in Synergetics. Springer, Heidelberg 1996).

[31] J. M. Raser and E. K. O'Shea, "Noise in gene expression: origins, consequences, and control", Science 309, 2010 (2005).

[32] M. B. Elowitz, A. J. Levine, E. D. Siggia and P. S. Swain, "Stochastic gene expression in a single cell", Science 297, 1183 (2002).

[33] M. A. Savageau, "Parameter sensitivity as a criterion for evaluating and comparing the performance of biochemical Systems", Nature 229, 542 (1971)

[34] U. Alon, M. G. Surette, N. Barkai and S. Leibler, "Robustness in bacterial chemotaxis", Nature 397, 168 (1999).

[35] N. Barkai and S. Leibler, "Robustness is simple biochemical networks", Nature 387, 913 (1997).

[36] J. A. G. M. de Visser, J. Hermisson, G. P. Wagner, L. Ancel Meyers, H. BagheriChaichian, J. L. Blanchard, L. Chao, J. M. Cheverud, S. F. Elena and W. Fontana, et al., "Perspective: Evolution and detection of genetic robustness", Evolution 57, 1959 (2003).

[37] J. U. Bowie, J. F. Reidhaar-Olson, W. A. Lim and R. T. Sauer, "Deciphering the message in protein sequences: tolerance to amino acid substitutions", Science $\mathbf{2 4 7}$, 1306 (1990).

[38] G. Giaever, A. M. Chu, L. Ni, C. Connelly, L. Riles, S. Veronneau, S. Dow, A. LucauDanila, K. Anderson and B. Andre, et al., "Functional profiling of the Saccharomyces cerevisiae genome", Nature 418, 387 (2002).

[39] Z. Gu, L. M. Steinmetz, X. Gu, C. Scharfe, R. W. Davis and W. Li, "Role of duplicate genes in genetic robustness against null mutations", Nature 421, 63 (2003).

[40] F. Li, T. Long, Y. Lu, Q. Ouyang, Ch. Tang, "The yeast cell-cycle network is robustly designed", Proc. Natl. Acad. Sci. (USA) 101, 4781 (2004)

[41] S. Bornholdt, K. Sneppen, "Robustness as an evolutionary principle", Proc. Roy. Soc. (London) B 267, 2281 (2000).

[42] Th. Handorf, O. Ebenhöh and R. Heinrich, "Expanding metabolic networks: scopes of compounds, robustness and evolution", J. Mol. Evol. 61, 498 (2005)

[43] R. Diestel, Graph Theory (Springer-Verlag New York 1997,2000). 
[44] B. A. Huberman and L. A. Adamic," Growth dynamics of the World-Wide Web", Nature 401, 131 (1999).

[45] S. Maslov and K. Sneppen, "Specificity and stability in topology of protein networks", Science 296, 910 (2002).

[46] R. Milo, S. Itzkovitz, N. Kashtan, R. Levitt, S. Shen-Orr, I. Ayzenshtat, M. Sheffer and U. Alon, "Superfamilies of designed and evolved networks", Science 303, 1538 (2004).

[47] R. Milo, S. Shen-Orr, S. Itzkovitz, N. Kashtan, D. Chklovskii and U. Alon, "Network motifs: simple building blocks of complex networks", Science 298, 824 (2002).

[48] S. S. Shen-Orr, R. Milo, S. Mangan and U. Alon, "Network motifs in the transcriptional regulation network of Escherichia coli", Nature Genetics 31, 64 (2002).

[49] S. Kirkpatrick, C. D. Gelatt and M. P. Vecchi, "Optimization by simulated annealing", Science 220, 4598, 671 (1983)

[50] T. Shibata and K. Fujimoto, "Noisy signal amplification in ultrasensitive signal transduction", Proc. Natl. Acad. Sci. U.S.A., 102, 331 (2005).

[51] M. Ipsen, A. S. Mikhailov, "Evolutionary reconstruction of networks", Phys. Rev. E 66, 046109 (2002).

[52] D. Cvetkovic̃, M. Doob, and H. Sachs, Spectra of Graphs: Theory and Applications (Johann Ambrosious Barth, Heidelberg, 1995) .

[53] P. Kaluza, M. Ipsen, M. Vingron and A. S. Mikhailov, "Design and statistical properties of robust functional networks: A model study of biological signal transduction", Phys. Rev. E 75, 015101 (2007).

[54] P. Kaluza and A. S. Mikhailov, "Evolutionary design of functional networks robust against noise", Europhys. Lett 79, 48001 (2007).

[55] P. Kaluza, H. Kori and A. S. Mikhailov, Evolutionary engineering of complex functional networks, in Managing Complexity, edited by D. Helbing (Springer, Berlin) 2007, pp 359-376. 